\title{
ELECTROSPUN ZEIN FIBERS AS CARRIERS TO STABILIZE (-)- EPIGALLOCATECHIN GALLATE
}

\author{
A Thesis \\ Presented to \\ The Faculty of Graduate Studies \\ of \\ The University of Guelph \\ by \\ YING LI
}

In partial fulfillment of requirements

For the degree of

Master of Science

September, 2009

(c) Ying Li, 2009 


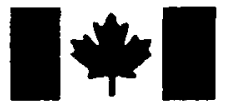

Library and Archives

Canada

Published Heritage

Branch

395 Wellington Street

Ottawa ON K1A ON4

Canada
Bibliothèque et

Archives Canada

Direction du

Patrimoine de l'édition

395, rue Wellington

Ottawa ON K1A ON4

Canada

Your file Votre référence

ISBN: 978-0-494-58368-5

Our file Notre référence

ISBN: 978-0-494-58368-5

NOTICE:

The author has granted a nonexclusive license allowing Library and Archives Canada to reproduce, publish, archive, preserve, conserve, communicate to the public by telecommunication or on the Internet, loan, distribute and sell theses worldwide, for commercial or noncommercial purposes, in microform, paper, electronic and/or any other formats.

The author retains copyright ownership and moral rights in this thesis. Neither the thesis nor substantial extracts from it may be printed or otherwise reproduced without the author's permission.
AVIS:

L'auteur a accordé une licence non exclusive permettant à la Bibliothèque et Archives Canada de reproduire, publier, archiver, sauvegarder, conserver, transmettre au public par télécommunication ou par l'internet, prêter, distribuer et vendre des thèses partout dans le monde, à des fins commerciales ou autres, sur support microforme, papier, électronique et/ou autres formats.

L'auteur conserve la propriété du droit d'auteur et des droits moraux qui protège cette thèse. $\mathrm{Ni}$ la thèse ni des extraits substantiels de celle-ci ne doivent être imprimés ou autrement reproduits sans son autorisation.
In compliance with the Canadian Privacy Act some supporting forms may have been removed from this thesis.

While these forms may be included in the document page count, their removal does not represent any loss of content from the thesis.
Conformément à la loi canadienne sur la protection de la vie privée, quelques formulaires secondaires ont été enlevés de cette thèse.

Bien que ces formulaires aient inclus dans la pagination, il n'y aura aucun contenu manquant.

\section{Canadä}




\section{ABSTRACT \\ ELECTROSPUN ZEIN FIBERS AS CARRIERS TO STABILIZE (-)- EPIGALLOCATECHIN GALLATE}

Ying Li

Advisor: Dr. Loong-Tak Lim

University of Guelph, 2009

In this research, a method was developed to continuously electrospin zeinethanol polymer solutions into ultrafine zein fibers. Scanning electron microscopy results showed that the morphology of zein fibers was affected by aqueous ethanol concentration, zein concentration, and the applied voltage. The electrospun zein fibers were tested to encapsulate (-)-epigallocatechin gallate (EGCG), a green tea polyphenol, under optimal electrospinning conditions. Zein fibers aged at $0 \% \mathrm{RH}$ for 1 day were found to achieve an enhanced EGCG retention ( $>98 \%$ ) in fibers when they were submerged in water. Fourier transform infrared spectroscopy results showed that hydrogen bonding and hydrophobic interactions were the major contributors involves in the stabilization of EGCG in zein fibers. The stability of EGCG in various aqueous solutions ( $\mathrm{pH} 4$ $-9,4-100^{\circ} \mathrm{C}$ ) was evaluated by using high performance liquid chromatography to determine the kinetics of degradation and epimerization reactions of EGCG. Pseudo first-order degradation kinetic models were developed for both reactions, of which the rate constants increased as the temperature and $\mathrm{pH}$ increased. Overall, the electrospun zein fibers enhanced considerably the stability of EGCG. Potentially, the electrospun zein fibers may be used as a carrier to stabilize the fortified EGCG in food. 


\section{Acknowledgements}

I would like to take this opportunity to deeply appreciate my advisor Dr. Loong-Tak Lim for his guidance, patience, professional advice, as well as countless support and encouragement throughout my M.Sc. study. I want to express my appreciation to my advisory committee members Dr. Yukio Kakuda and Dr. Yoshinori Mine for their contributions on my research work and thesis adjustment writing. I am very grateful to Dr. Sandy Smith for her technical advice on SEM measurements.

Special thanks to Natural Sciences and Engineering Research Council of Canada (NESRC) and H.J. Heinz Company of Canada Ltd. for their essential financial support, as well as to Mitsui Norin Company Ltd. for its donation of (-)Epigallocatechin Gallate. I would like to thank Dr. Yukio Kakuda and Dr. Robert Lencki for their generosity providing materials and laboratory facilities. Many thanks to my Packaging and Biomaterials laboratory members: Ana Cristina Vega Lugo, Yucheng Fu, Solmaz Alborzi, Yuting Zhou, Suramya Minhindukulasuriya, Roc Chan, Grace Wong, Alex Jensen and Che Lam and Qian Xiao for their assistance, friendship, tolerance and patience.

I would like to express my deepest gratitude to my beloved parents Dawei Li and Xirong Yang for their infinite love, unconditional support and encouragement throughout my life. Last but not the least, I strongly thank my boyfriend Christopher Carl Rogers for his unfailing love, patience and support on my study. 


\section{Table of Contents}

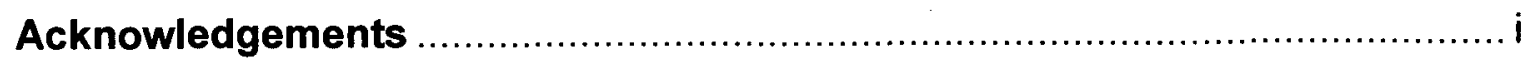

Table of Contents

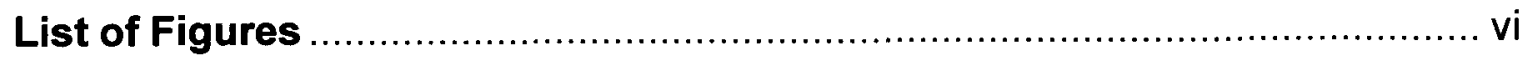

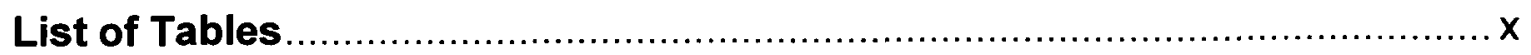

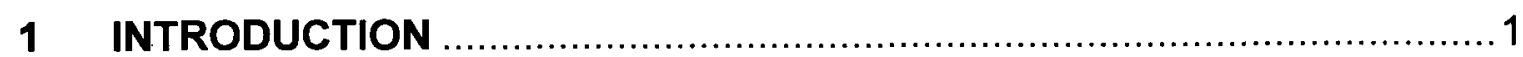

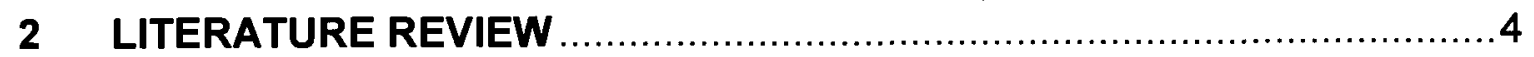

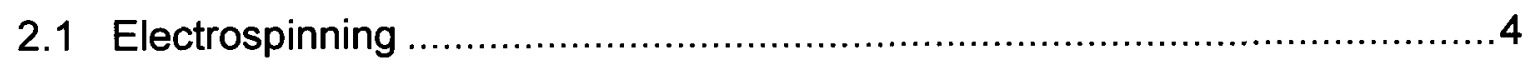

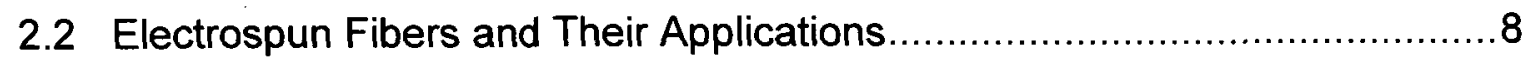

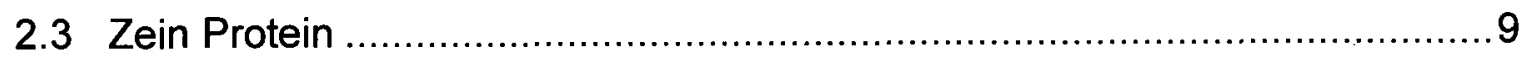

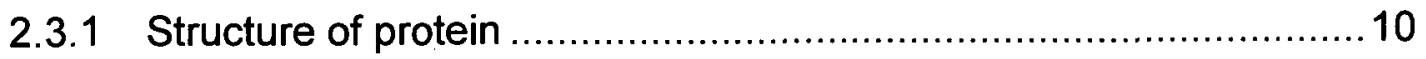

2.3.2 Function and applications of zein protein ...................................11

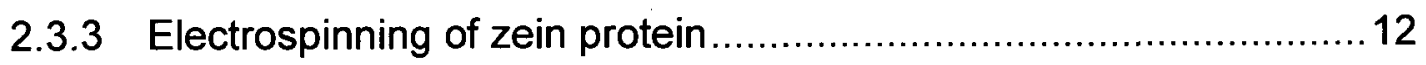

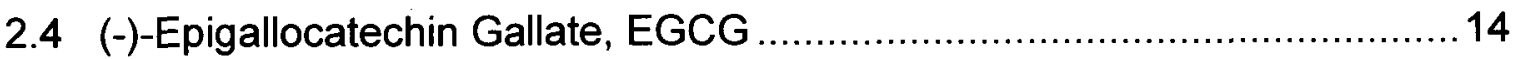

2.5 Nature of Polyphenol-Protein Interactions …........................................ 17

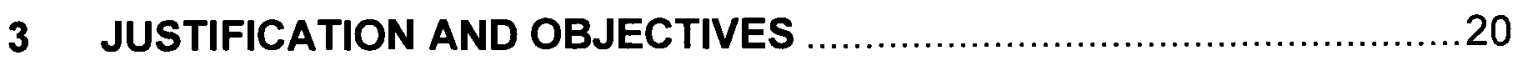

4 ELECTROSPINNING OF ZEIN PROTEIN FIBERS …........................21

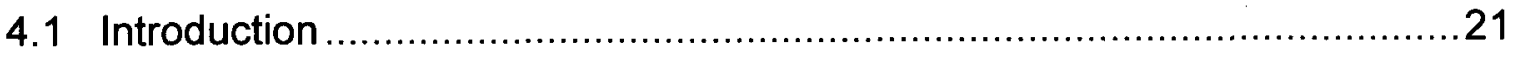

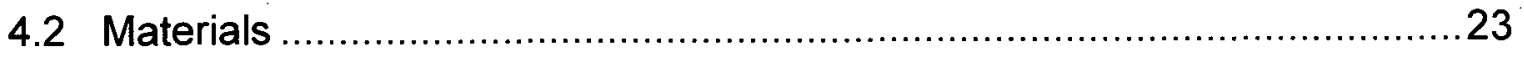

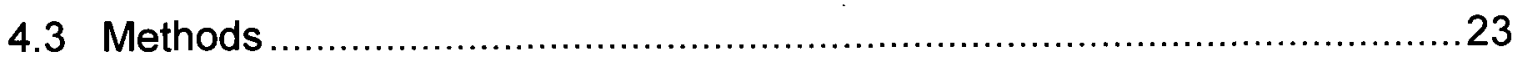

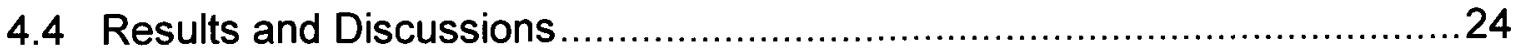

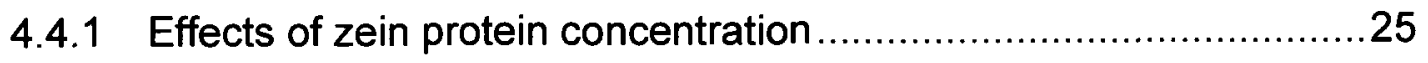




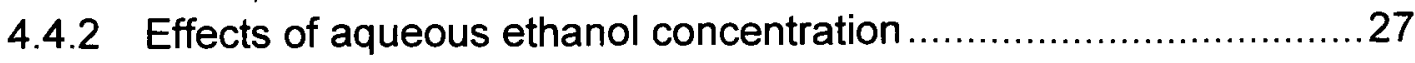

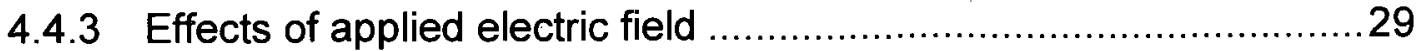

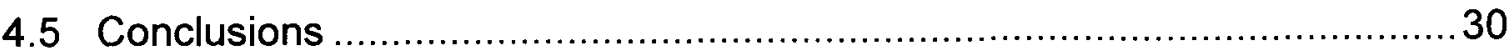

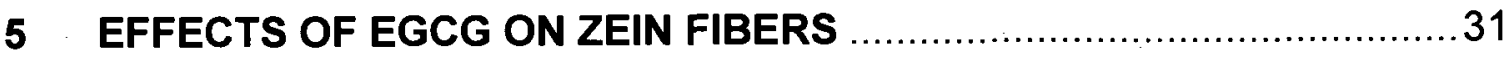

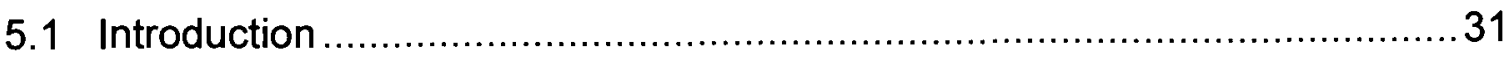

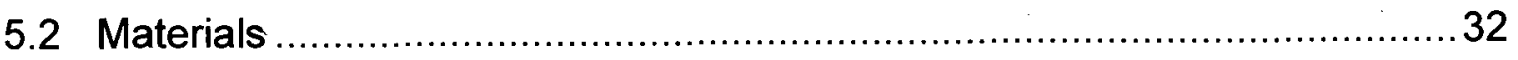

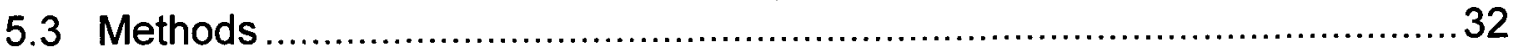

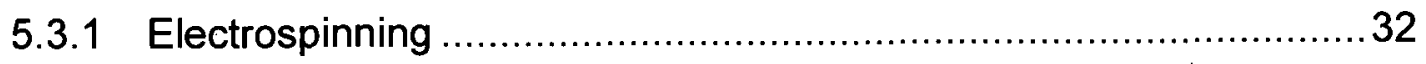

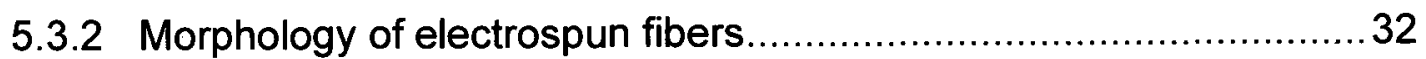

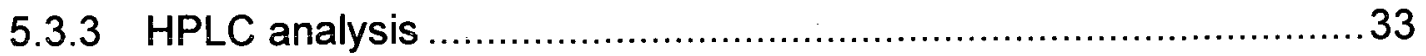

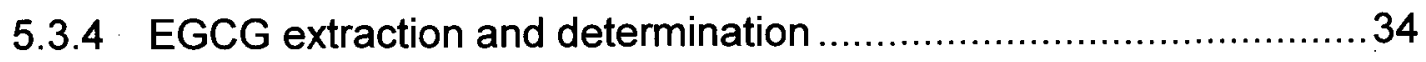

5.3.5 Fourier transform infrared (FTIR) evaluation...............................36

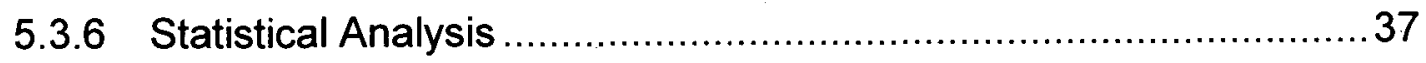

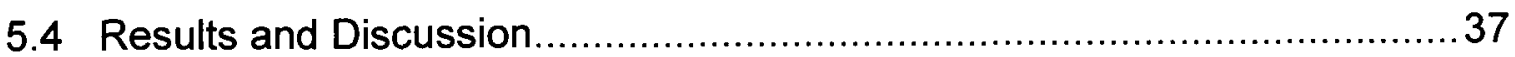

5.4.1 Effects of EGCG on morphology of zein fibers ............................. 37

5.4.2 Previous stability study of EGCG in zein fibers.............................38

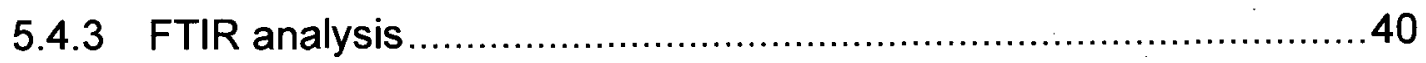

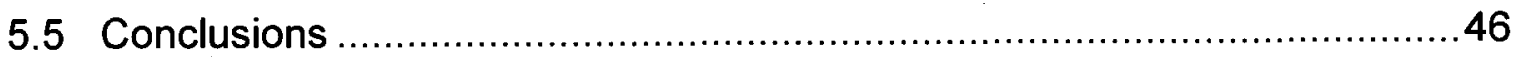

6 THE STABILITY OF EGCG ENCAPSULATED IN ELECTROSPUN ZEIN

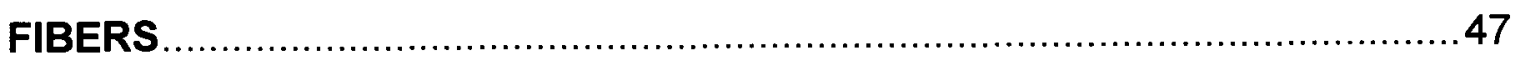

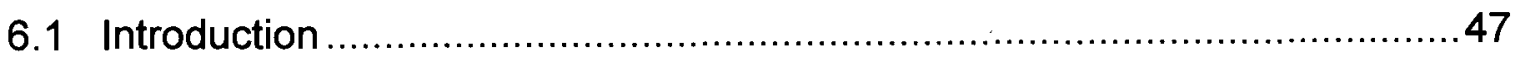

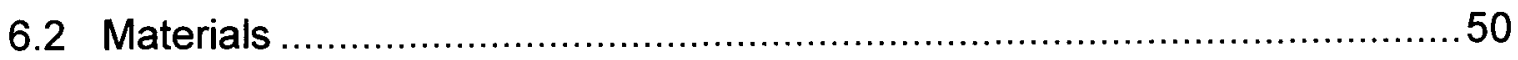

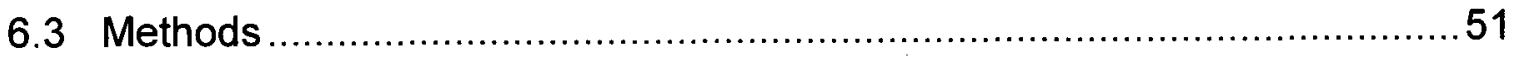




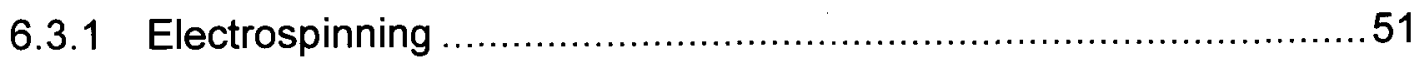

6.3.2 Morphology of electrospun fibers ...........................................51

6.3.3 Preparation of the aqueous model systems ...............................51

6.3.4 Experimental design for stability studies ....................................51

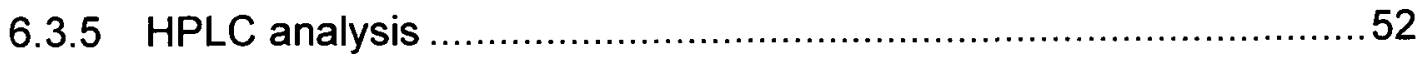

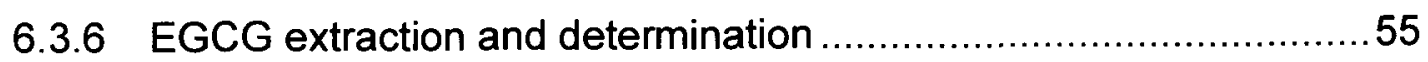

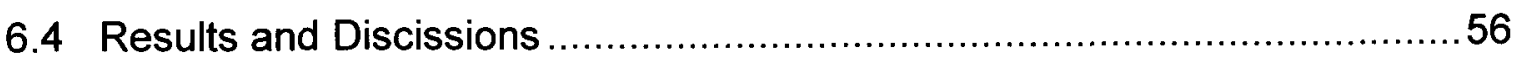

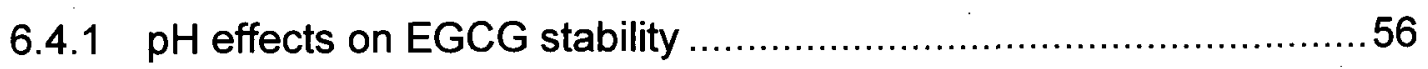

6.4.2 Temperature effects on EGCG stability .....................................58

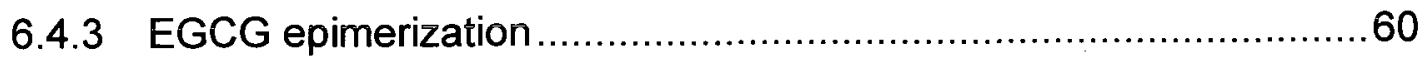

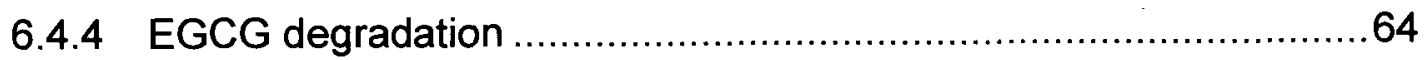

6.4.5 Diffusion of EGCG into the aqueous solution.............................66

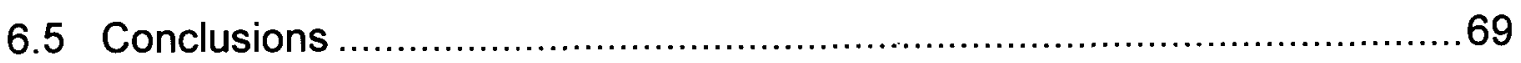

7 KINETIC MODELLING OF EGCG DEGRADATION ..............................

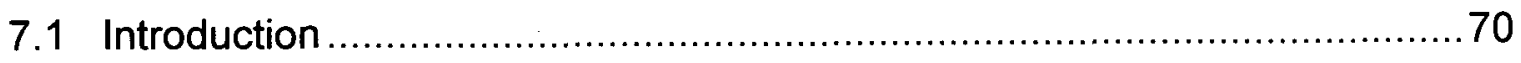

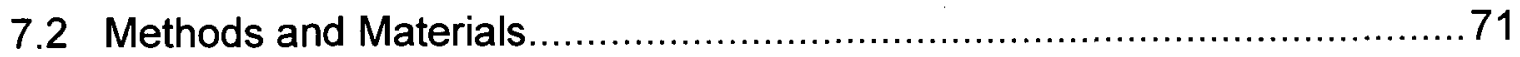

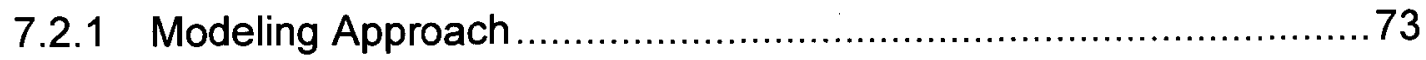

7.2.2 Fitting of kinetic models to experiment data................................76

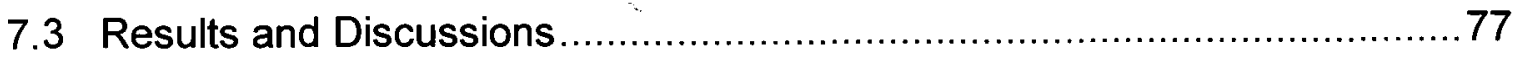

7.3.1 Verification of the pseudo first-order kinetics for the degradation of

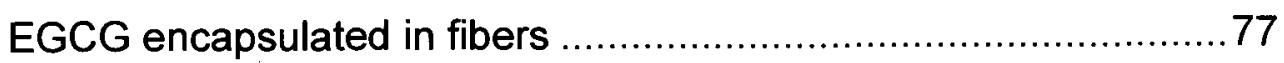

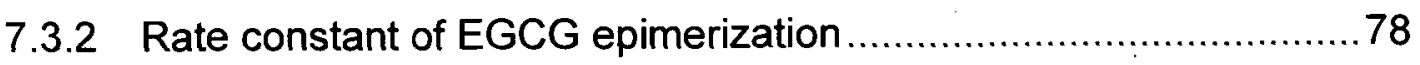

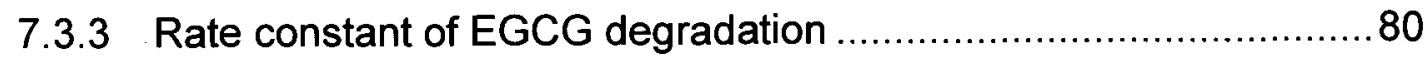




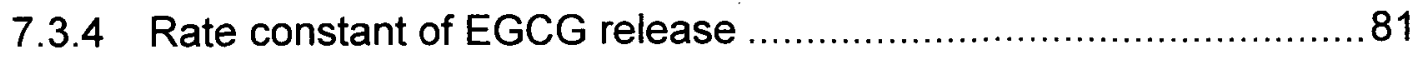

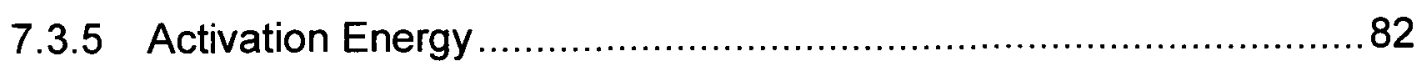

7.3.6 Turning point temperature in the reaction kinetics of EGCG ..........85

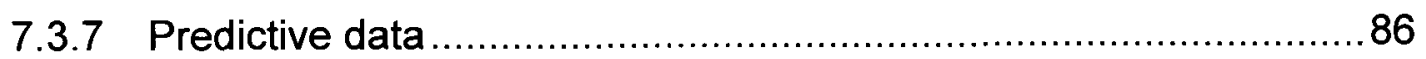

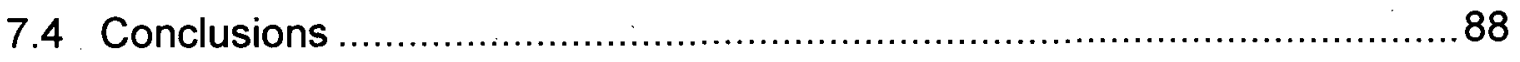

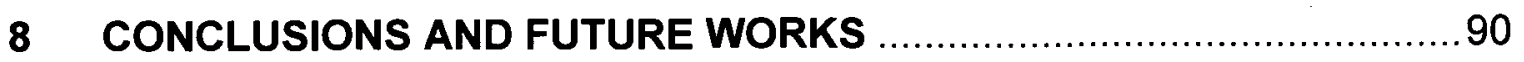

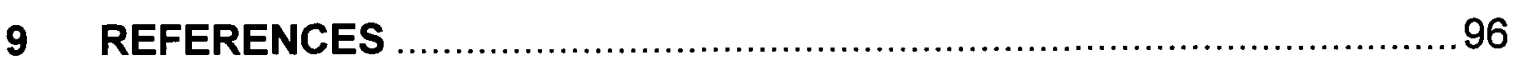

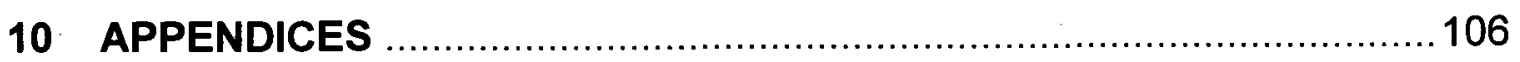

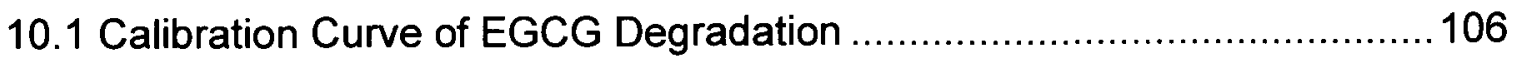

10.2 Calibration Curve of GCG Degradation................................................ 106

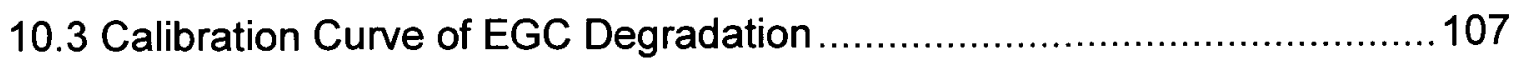

10.4 Matlab Program for model fitting and rate constant determination...........107

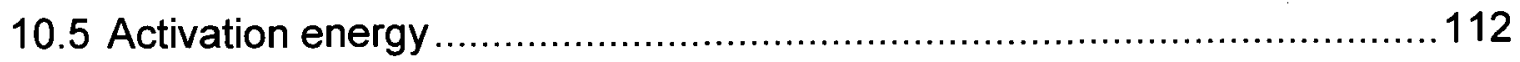

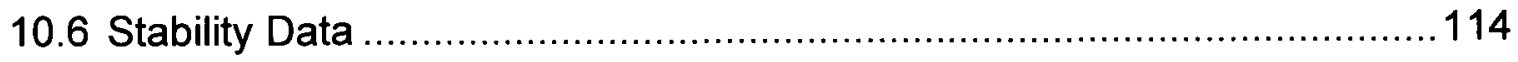




\section{List of Figures}

Figure 1 - Electrospinning process and setup (Li and others 2009).

Figure 2 - Average diameters of zein fibers electrospun from aqueous ethanol solutions of different zein concentrations with an ethanol/water ratio of 70:30 (v/v) in an electric field of $2 \mathrm{kV} / \mathrm{cm}$ (Yao and others 2007).

Figure 3 - Schematic representation for epimerization and degradation of EGCG (Wang and others 2008).

Figure 4 - Interaction produced when various concentrations of gliadin and catechin were combined in $0.02 \mathrm{M}(\mathrm{pH} 4.2)$ potassium phosphate buffer and heated for $30 \mathrm{~min}$ at $80^{\circ} \mathrm{C}$ (Siebert and others 1996). 19

Figure 5 - SEM images of zein fibers electrospun at $15 \mathrm{kV}$ in $70 \%$ ethanol at various zein concentrations. A: $10 \%$ zein $(276 \pm 26 \mathrm{~nm})$; B: $20 \%$ zein (386 \pm 32 $\mathrm{nm})$; C: $30 \%$ zein $(462 \pm 43 \mathrm{~nm})$. 26

Figure 6 - Average diameters of zein fibers electrospun from different zein concentrations dissolved in $70 \%$ aqueous ethanol $(w / w)$ under an electric field of $15 \mathrm{kV}$ .27

Figure 7 - SEM images of zein fibers electrospun at $15 \mathrm{kV}$ at $20 \%$ zein dissolved in various ethanol concentrations. A: $60 \%$ ethanol ( $369 \pm 32 \mathrm{~nm}$ ); B: $70 \%$ ethanol (402 $\pm 37 \mathrm{~nm})$; C: $80 \%$ ethanol $(518 \pm 47 \mathrm{~nm})$; D: $90 \%$ ethanol $(683 \pm 62 \mathrm{~nm})$. .28

Figure 8 - Average diameters of zein fibers electrospun from polymer solutions of different ethanol/ water ratios of $60: 40 ; 70: 30 ; 80: 20 ; 90: 10(\mathrm{w} / \mathrm{w})$ under an electric field of $15 \mathrm{kV}$. 29

Figure 9 - SEM images of 20\% zein polymer dissolved in $70 \%$ ethanol concentration at various voltages. A: $15 \mathrm{kV}(347 \pm 27 \mathrm{~nm})$; B: $20 \mathrm{kV}(311 \pm 23$ $\mathrm{nm})$. 
Figure 10 - Schematic representation of EGCG extraction process.

Figure 11 - Schematic representation of effects on EGCG aging and humidity process.

Figure 12 - SEM images of electrospun fibers (20\% zein; $70 \% \mathrm{w} / \mathrm{w}$ ethanol) before and after immersion in water for $30 \mathrm{~min}$. A: zein fibers before water immersion (386 $\pm 32 \mathrm{~nm}$ ); B: zein + EGCG before water immersion (472 \pm 46 $\mathrm{nm})$; C: zein fibers after water immersion ( $457 \pm 38 \mathrm{~nm}$ ); D: zein + EGCG after water immersion $(631 \pm 56 \mathrm{~nm})$. 38

Figure 13 - Effects of aging, relative humidity, and extraction time on percent residual EGCG from electrospun zein fibers after immersing in water for up to 60 $\min$. 39

Figure 14 - Representative FTIR spectra for zein fibers alone (A), EGCG powder (B), and zein fibers with 20\% EGCG (C). The spectra for fiber samples were taken immediately after electrospinning before aging (at day 0 ). The fitted curves were based on the peak positions determined from second-derivative of the spectrum followed by Gaussian curve fitting for protein and Lorentzian fitting for EGCG.

Figure 15 - FTIR spectra for zein and EGCG-containing zein fiber aged in $0 \%$ or $75 \% \mathrm{RH}$ conditions at $21 \pm 2{ }^{\circ} \mathrm{C}$. A: zein fibers at $0 \% \mathrm{RH}$; B: zein fibers at $75 \%$ $\mathrm{RH}$; C: zein + EGCG fibers at 0\% RH; D: zein + EGCG fibers at $75 \% \mathrm{RH}$. 44

Figure 16 - FTIR spectra and deconvoluted bands for EGCG-containing zein fibers aged in $0 \% \mathrm{RH}$ conditions for 3 days at $21 \pm 2{ }^{\circ} \mathrm{C}$. 45

Figure 17 - Chemical structures of green tea catechins: (-)-epigallocatechin gallate (EGCG), (-)-gallocatechin gallate (GCG), (-)-epigallocatechin (EGC) and (-)-gallocatechin (GC) (Wang and others 2008). 48

Figure 18 - HPLC profile of EGC, EGCG and GCG standards was detected at $274.5 \mathrm{~nm}$ respectively. .53 
Figure 19 - Schematic representation of EGCG extraction process for the stability study. 56

Figure 20 - Remaining percentage of residual EGCG encapsulated by electrospun zein fibers at $25{ }^{\circ} \mathrm{C}$ in various $\mathrm{pH}$, compared to percentage of controls. A: $\mathrm{pH}$ 9; $\mathbf{B}: \mathrm{pH} 7$; C: $\mathrm{pH} 5$ and D: $\mathrm{pH} 3$. 58

Figure 21 - Remaining percentage of residual EGCG encapsulated by electrospun zein fibers in aqueous system with $\mathrm{pH} 7$ at different temperature, compared to the percentage of controls. A: $100{ }^{\circ} \mathrm{C}$; B: $60^{\circ} \mathrm{C}$; C: $25^{\circ} \mathrm{C}$ and D: 4 ${ }^{\circ} \mathrm{C}$. 60

Figure 22 - Perce nt remaining of residual EGCG and its epimer, GCG, in electrospun zein fibers as compared with the percentage of EGCG control and its epimer under various $\mathrm{pH}$ and temperature conditions: $\mathrm{A}$ : EGCG at $\mathrm{pH} 9$ and $4{ }^{\circ} \mathrm{C}$; B: GCG at pH 9 and $4{ }^{\circ} \mathrm{C}$; C: EGCG at pH 9 and $100^{\circ} \mathrm{C}$; D: GCG at pH 9 and $100^{\circ} \mathrm{C}$; E: EGCG at pH 3 and $100^{\circ} \mathrm{C}$ and F: GCG at pH 3 and $100^{\circ} \mathrm{C}$.

Figure 23 - Percent remaining of residual EGCG encapsulated by electrospun zein fibers and its degraded compounds, EGC, compared to the percentage of EGCG control and its degraded compounds in various conditions. A: EGCG under $\mathrm{pH} 9$ at $4{ }^{\circ} \mathrm{C}$; $\mathrm{B}$ : EGC under $\mathrm{pH} 9$ at $4{ }^{\circ} \mathrm{C}$; C: EGCG under $\mathrm{pH} 9$ at $100^{\circ} \mathrm{C}$; D: EGC under $\mathrm{pH} 9$ at $100{ }^{\circ} \mathrm{C}$; E: EGCG under $\mathrm{pH} 3$ at $100^{\circ} \mathrm{C}$ and $\mathrm{F}$ : EGC under $\mathrm{pH} 3$ at $100^{\circ} \mathrm{C}$. 65

Figure 24 - The percentage of residual EGCG encapsulated by electrospun zein fibers (A) and EGCG diffused from fibers into the aqueous solution (B) at $\mathrm{pH} 7$ at two temperatures.

Figure 25 - The percentage of residual EGCG encapsulated by electrospun zein fibers (A) and diffused EGCG from fibers (B) at $60^{\circ} \mathrm{C}$ in aqueous system with $\mathrm{pH}$ 5 and 9. 68

Figure 26 - Schematic representation of EGCG reactions. .72 
Figure 27 - Arrhenius plots of apparent first-order degradation of EGCG encapsulated in electrospun zein fibers at various temperatures in aqueous solutions with $\mathrm{pH} 9$.

Figure 28 - Arrhenius plots of the rate constants $\left(k_{1}\right)$ for epimerization reactions of EGCG immobilized in electrospun fibers in aqueous system under different $\mathrm{pH}$ conditions.

Figure 29 - Arrhenius plots of the rate constants for the degradation reactions of EGCG + GCG immobilized in electrospun fibers in aqueous system under different $\mathrm{pH}$ conditions.

Figure 30 - Arrhenius plots of the rate constants $\left(k_{2}\right)$ of the EGCG diffused from electrospun fibers in aqueous system under different $\mathrm{pH}$ conditions.

Figure 31 - Arrhenius plots of rate constant $\left(k_{1}\right)$ for the epimerization of from EGCG to GCG in pH $3-9$ aqueous systems at various temperatures, ranged from $4-100^{\circ} \mathrm{C}$. 86

Figure 32 - Modeled and experimental values of residual EGCG in various aqueous systems. RMSE ranged from 0.85 to 2.51 and $R^{2}>0.95$.

Figure 33 - Calibration curve of EGCG standard was linear over the range of $0.02-0.1 \mathrm{mg} / \mathrm{mL}$ 106

Figure 34 - Calibration curve of GCG standard. 106

Figure 35 - Calibration curve of EGC standard. 107 


\section{List of Tables}

Table 1 - Factors affecting the properties of electrospun fibers (Vega-Lugo and Lim 2008a) 7

Table 2 - Activation energy $\left(E_{a}\right)$ for reaction rate constants of EGCG encapsulated in fibers and EGCG controls in $\mathrm{pH} 3-9$ aqueous systems at various temperatures, ranged from $4-100^{\circ} \mathrm{C}$.

Table 3 - Remaining percentage of residual EGCG encapsulated by electrospun zein fibers in aqueous system with $\mathrm{pH} 3-9$ at different temperature, compared to the percentage of controls.

Table 4 - Remaining percentage of epimerized GCG from EGCG encapsulated by electrospun zein fibers in aqueous system with $\mathrm{pH} 3-9$ at different temperature, compared to the percentage of GCG epimerized from EGCG controls. 115

Table 5 - Remaining percentage of degraded EGC from EGCG encapsulated by electrospun zein fibers in aqueous system with $\mathrm{pH} 3-9$ at different temperature, compared to the percentage of EGC degraded from EGCG controls. 116 


\section{INTRODUCTION}

Food processing techniques are important for rendering many foods safe for consumption and for imparting desirable changes such as protein denaturation, textural softening and aroma generation. Nevertheless, various food processing methods can also induce the degradation of bioactive compounds (micronutrients and nutraceuticals) in foods, especially those compounds that are sensitive to oxygen, water and heat (Mckillop and others 2002; Nguyen and others 2003; Akanbi and Oludemi 2004; Nisha and others 2005). Food products can be fortified to make up for the expected process loss, but the added active compounds may react with other food components, potentially reducing overall nutritional and organoleptic qualities (Salgueiro and others 2002; Xia and Xu 2005). The bioavailability of these active compounds can also be affected by their binding with the food matrix, the form of nutrient derivative available, and the gastrointestinal conditions that the compounds are subjected to (Ristow and others 1982; Hurrell 1997; Brouwer and others 2001; Basu and Donaldson 2003). To circumvent these problems, the fortified active compounds often need to be protected, using techniques such as spray-drying microencapsulation, extrusion encapsulation, fluidized bed coating, coacervation, spray chilling, molecular inclusion using $\beta$-cyclodextrin, protein precipitation, liposome entrapment and hydrogel entrapment (Gibbs and others 1999; Remondetto and others 2004; Yuliani and others 2004). These methods typically produce capsules of several microns to millimeters in size. 
Electrospinning is a process that uses electric fields to spin fibers with diameters ranging from hundreds to tens of nanometers. Because of their submicron diameter and large surface-to-volume ratio, electrospun fibers tend to exhibit enhanced interactions with their surrounding medium, making them an ideal material for sensors, controlled release systems, enzyme immobilization, and tissue engineering applications (Huang and others 2003; Ko 2003; Li and Xia 2004). Because heat is absent during electrospinning and the process is relatively simple, electrospun fibers may serve as innovative carriers to deliver bioactive components in foods. In the present study, we exploit the hydrophobic properties of zein and an electrospinning technique to stabilize (-)epigallocatechin-gallate (EGCG), a plant polyphenol found mainly in tea. Zein was chosen for this study because it is a prolamin, which is insoluble in water due to the presence of large amount of hydrophobic amino acid residues such as leucine, proline, alanine, and phenylalanine (Gianazza and others 2003). In highmoisture foods, water-insoluble fibers are expected to be more optimal for protecting the encapsulated compound than fibers electrospun from watersoluble polymers. On the other hand, EGCG was chosen for this study due to its antioxidative properties, antimicrobial activities, and health benefits (LandisPiwowar and others 2007).

Proteins have been known to interact strongly with polyphenol through hydrogen bonding and hydrophobic interactions. Madhan and others (2005) reported that although there were no major changes in the secondary structure of collagen after treatment with catechin, the treated collagen resisted solubilization 
in urea and shrinkage upon exposure to elevated temperatures. They suggested that hydrogen bonding and hydrophobic interactions are the driving forces contributing to the stabilization of collagen by catechin. Siebert and others (1996) showed that heating gliadin samples in the presence of polyphenol led to an increase in haze, and speculated that hydrophobic interaction played a more important role than hydrogen bond. In the study involving the interaction between EGCG and human serum albumin, Maiti and others (2006) postulated that hydrophobic interaction is more prominent during initial association, while van der Waals interactions and hydrogen bonding are important for stabilizing the complex. EGCG is not stable under alkaline conditions and may undergo oxidation, polymerization and epimerization during thermal processing (Zhu and others 1997; Sang and others 2005; Wang and others 2006; Yilmaz 2006). In view of the propensity of protein to interact with polyphenols, we hypothesize that encapsulating EGCG in electrospun zein fibers may result in enhanced EGCG stability.

Using EGCG as a model bioactive compound, the objectives of this study were to develop a method to electrospin zein fibers to encapsulate EGCG, and to evaluate the stability of the encapsulated EGCG in water. Here, we introduced an inverted electrospinner setup to enable continuous spinning of zein fibers and evaluated the morphology of electrospun zein ultrafine fibers using various solution parameters and process conditions. We also conducted preliminary studies to determine the stability of EGCG in zein fibers in an aqueous model. EGCG in zein electrospun fiber may result in enhanced EGCG stability. 


\section{LITERATURE REVIEW}

\subsection{Electrospinning}

Electrospinning is a process that uses an electric field to control the formation and deposition of polymeric fibers whose diameters range from micro $\left(10^{-6} \mathrm{~m}\right)$ to nanoscale $\left(10^{-9} \mathrm{~m}\right)$. In a typical electrospinner set-up (Figure 1$)$, the polymer solutions are electrospun by using the stainless steel spinneret which is attached to the positive electrode of a direct current (DC) power supply, while the collector plate is grounded. The spinneret is connected through tubing to a plastic syringe containing the polymer solution, which is fed by means of an infusion pump. As the voltage is increase to the critical level, the polymer solution is jetted as a stream of fiber through the Taylor cone, when the electrostatic charge accumulated on the surface of the Taylor cone overcomes the tension of the polymer solution. Due to the rapid evaporation of solvent from the polymer jet, a solidified polymer is formed and drawn into a continuous thin fiber as it undergoes whipping motion in the air. As the fiber continues to deposit on the collector plate, a nonwoven fibrous mat is formed (Reneker and others 2000; Matthews and others 2002; Vega-Lugo and Lim 2008b; Li and others 2009). 


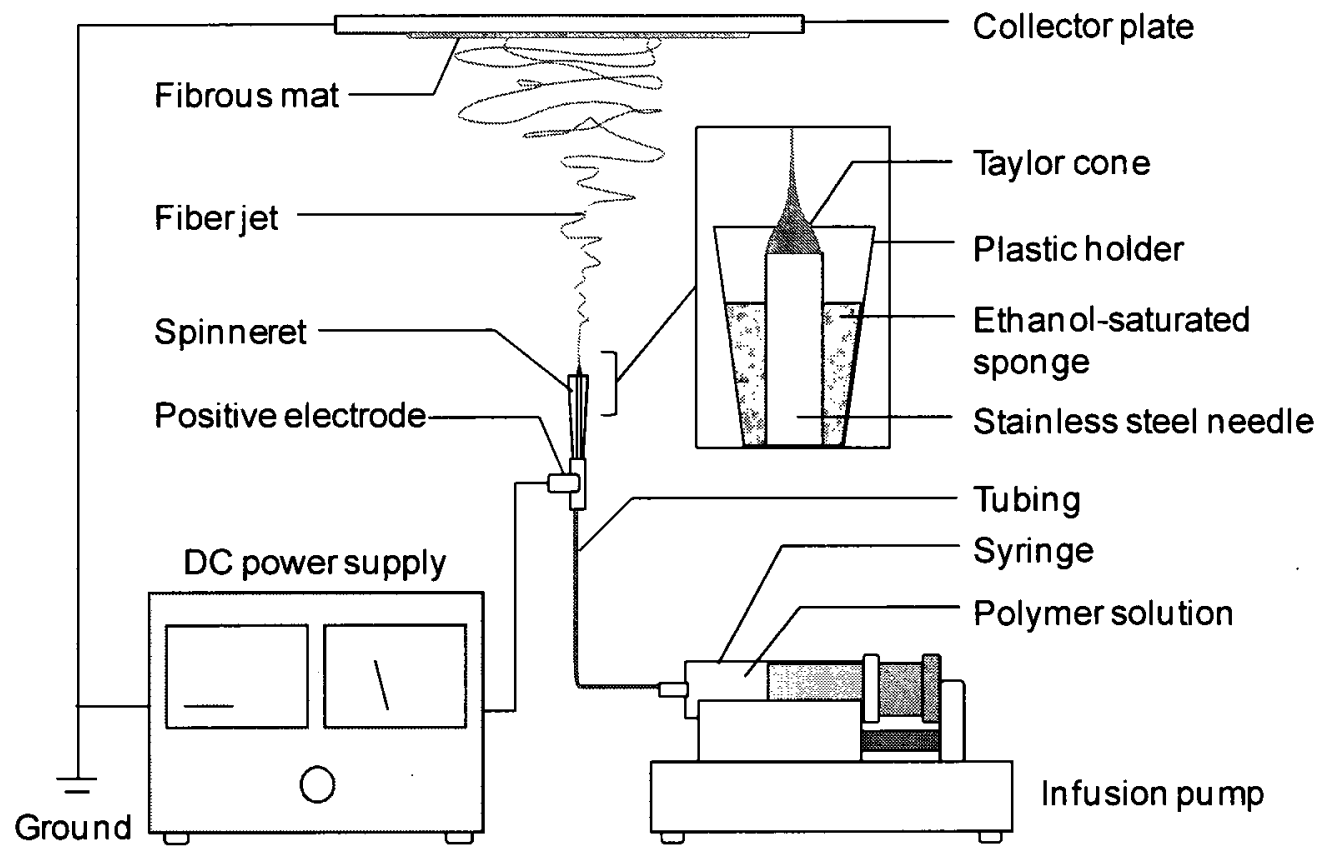

Figure 1 - Electrospinning process and setup (Li and others 2009).

The effects of processing conditions and solution properties on electrospun fibers have been summarized by Vega-Lugo and Lim (2008a) in Table 1. As shown, the properties of solution, electrospinning conditions and ambient parameters all significantly contribute to the properties of the resulting electrospun fibers such as morphology, diameter, and their mechanical characteristics. One of the most important factors influencing the diameter of electrospun fibers is viscosity, which is related to polymer molecular weight and solution concentration. For example, as the viscosity of solution increases, the diameter of fibers becomes larger, the beads become bigger and their shape changes from rotund to spindle-like (Fong and others 2002; Zhang and others 2005; Vega-Lugo and Lim 2008a). The voltage applied to the spinneret also plays a role in affecting the morphology of electrospun fibers. It has been 
reported that increasing the applied voltage results in decreased stability of the solution jet, which, in turn, increases the amount of beads formed and fiber diameter (Deitzel and others 2001). The longer the spinneret-collector distance, the greater the occurrence of beads on the collector because the field strength then exceeds its optimal value (Megelski and others 2002; Jarusuwannapoom and others 2005). In some circumstances, the increased distance leads to smaller diameter fibers since there is a longer wiping time for the solution to be stretched before it is deposited on the target (Reneker and others 2000; Zhao and others 2004; Ayutsede and others 2005). 
Table 1 - Factors affecting the properties of electrospun fibers (Vega-Lugo and Lim 2008a).

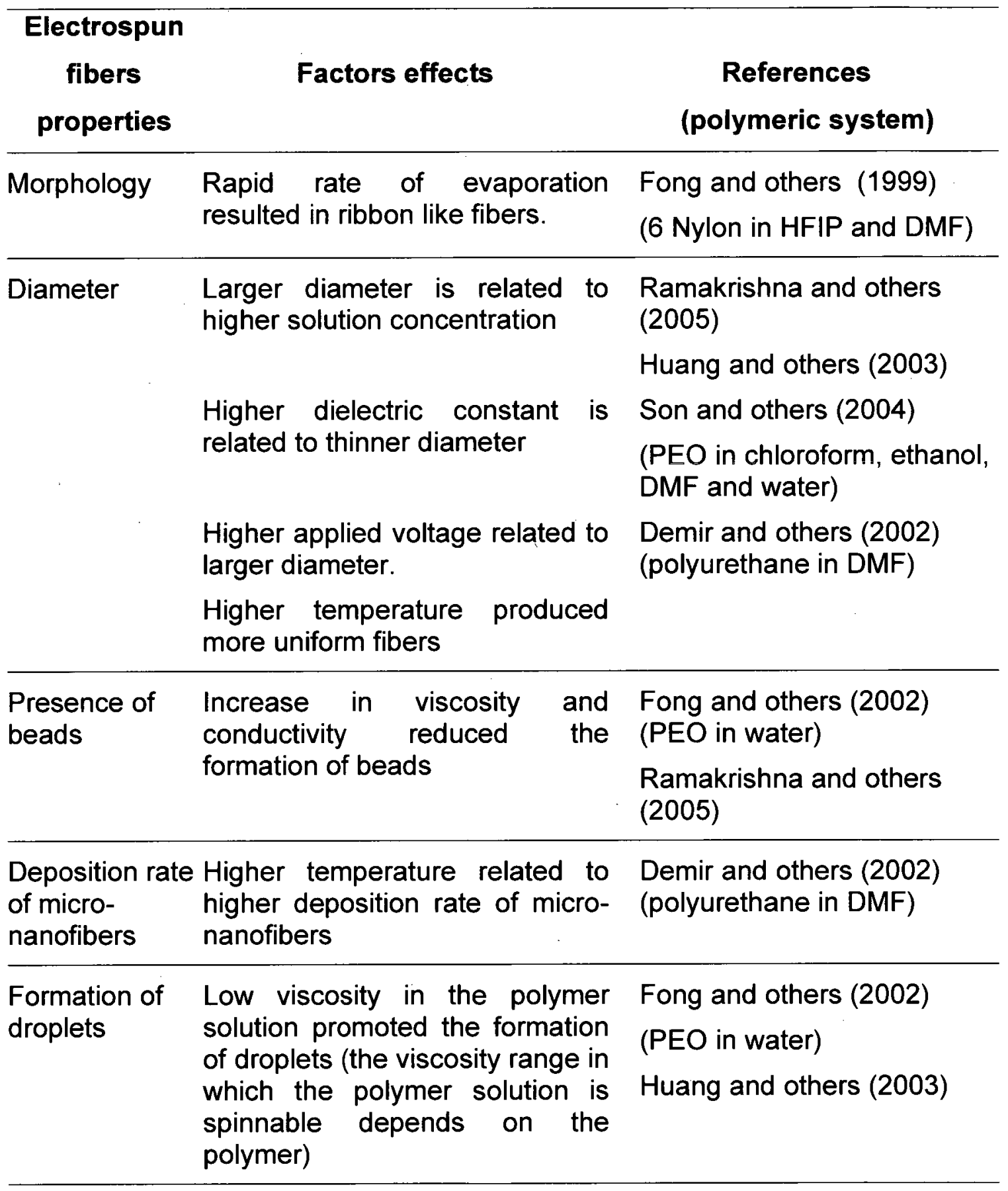




\subsection{Electrospun Fibers and Their Applications}

Although Formhals first patented the method of electrospinning in 1934 , it has not received much attention until recently (Miyoshi and others 2005). Because of their sub-micron diameter and large surface-to-volume ratio, electrospun fibers tend to exhibit enhanced interactions with their surrounding medium, making them an ideal material for biosensors, controlled release systems, enzyme immobilization, wound dressings, nanocomposites for dental restoration and tissue engineering applications (Zong and others 2002; Ko 2003; Huang and others 2003; Li and Xia 2004; Zhang and others 2005; Chew and others 2006). In this area, some researchers have already reported how electrospinning can be used to fabricate scaffolds of synthetic, biodegradable polymers (Stitzel and others 2001). Matthews and other coworkers have successfully adapted the method to produce tissue-engineering scaffolds composed of collagen fibers (Matthews and others 2002). A variety of electrospinning experiments have been carried out with different kinds of protein such as wheat protein, zein protein and soy protein, at different solution concentrations to illustrate the interplay between protein structure and the characteristics of the electrospinning process (Yao and others 2007).

One of the most important applications of electrospun fiber is as a carrier for medically active compounds in drugs. For instance, Kim and other researchers adopted the electrospun poly (lactide-co-glycolide) fiber as a carrier for hydrophilic cefoxitin sodium, an antibiotic drug. The drug was successfully incorporated and released from nanofiberous scaffolds without the loss of 
structural integrity or change in functionality (Zong and others 2002). Chew and others demonstrated that the electrospinning process could be used effectively to encapsulate different bioactive agents (Chew and others 2006). In their study, they adopted drug-encapsulated electrospun fibers for controlled release of low molecular weight drugs, plasmid DNA, proteins and glycosaminoglycan salts. In another study, biodegradable poly(L-lactic acid) (PLLA) electrospun fibers were applied as a carrier for the controlled release of various surfactants (cationic, anionic and nonionic) and typical drugs, rifampicini (Zeng and others 2003).

In our present study, a similar electrospinning technique will be adopted to produce fibers as a carrier for incorporating a polyphenol nutraceutical to improve its stability through physical immobilization and chemical interaction with a biopolymer.

\subsection{Zein Protein}

Due to the high ratio of nonpolar amino acid residues and lack of basic amino acids, prolamin proteins, such as corn zein and wheat gliadin, are soluble in aqueous alcohol, but not in water. This makes them an ideal carrier for encapsulating bioactive agents in high moisture food products. As one of the common components in food, the use of alcohol as a solvent for electrospinning is also advantageous because it can vaporize readily from the fiber as the polymer jet takes flight in the air, forming dry and solid fibers upon arriving at the collection target. Therefore, corn zein was chosen for our research. 


\subsubsection{Structure of protein}

Generally, proteins are characterized by their three-dimensional organization as primary, secondary, tertiary, and quaternary structures. Secondary structure in proteins consists of local inter-residue interactions mediated by hydrogen bond and hydrophobic interactions. The most common secondary structures are $\alpha$-helices and $\beta$-sheets (Fersht 1999).

Zein is in the class of prolamin from corn, and is an abundant protein in corn gluten meal, a coproduct of corn wet milling (Shukla and Munir 2001). Biologically, zein is a mixture of proteins varying in molecular size $\left(M_{r} 17,000-\right.$ $27,000)$. It is classified into $\alpha, \beta, \gamma$, and $\delta$ zeins according to their solubility in alcohol. Its solubility in alcohol is mainly due to a zein which represents in more than $70 \%$ of the total zein (Mejia and others 2007). Zein has a large amount of hydrophobic residues such as leucine, proline, alanine, and phenylalanine (Gianazza and others 1977), which explains its significant hydrophobic properties. Because it is insoluble in water, most structural studies are performed in alcohol solutions. Forato and others demonstrated that the secondary structure of zein prepared with and without solubilization in alcohol, when analyzed in the solid state by FTIR and solid-state ${ }^{13} \mathrm{C}-\mathrm{NMR}$ and by circular dichroism (CD) in an alcohol solution, was not affected by alcohol solubilization (Forato and others 2003). When dissolved in aqueous alcohol, the protein is known to be rich in ahelical content (50-60\%) with turn or random coil configurations comprising the remaining structure (Argos and others 1982). The amide I and II bands are the two most prominent vibrational bands of the protein backbone. Mejia and other 
researchers investigated the formation of $\alpha$ helices and $\beta$ sheets by examining the amide I region of $1700-1600 \mathrm{~cm}^{-1}$ by using Fourier Transform IR (FTIR) spectroscopy, which was due almost entirely to the $C=O$ stretch vibrations. The amide II band in $1600-1500 \mathrm{~cm}^{-1}$ was derived mainly from $\mathrm{N}-\mathrm{H}$ bending and from the $\mathrm{C}-\mathrm{N}$ stretching vibrations. Their conclusions are in the agreements with the findings from Krimm and Bandekar (1986), Ismail and others (1992), Jackson and Mantsh (1995) and Panick and others (1999).

\subsubsection{Function and applications of zein protein}

Zein is industrially interesting due to its ability to form tough, glossy, hydrophobic grease-proof coatings and their resistance to microbial attack. Potential applications of zein include use in fiber, adhesive, coating, ceramic, ink, chewing gum and biodegradable plastics (Shukla and Munir 2001). Zein has the additional benefits of being renewable and biodegradable and has also been used in the pharmaceutical industry to coat capsules, protect drugs, control release and mask of flavors and aromas (Shukla and Munir 2001). Zein microspheres have been investigated for use as carriers to protect drugs from stomach acid (Yao and others 2007). Dong and other researchers reported that zein films as matrices show excellent ability to provide the proliferation and attachment for both human liver cells and murine fibroblast cells, indicating that zein is a promising biomaterial with good biocompatibility for the development of tissue engineering (Dong and others 2004). Because zein is hydrophobic, it has been used in the food industry as a moisture barrier for candies, rice, dried fruits and nuts. Zein has been investigated for use as a structural material in packaging 
applications because of its film-formation properties. In particular, zein forms tough and glossy films and coatings resistant to microbial attacks which can function as an oxygen, lipid and moisture barrier. Zein has also been investigated for its fiber forming properties (Miyoshi and others, 2005). These studies suggest that zein is a promising biomaterial which can be applied in various areas.

\subsubsection{Electrospinning of zein protein}

Various proteins have been previously electrospun into fibers such as whey protein, soy protein, zein protein, collagen and fibrinogen (Matthews and others 2002; Woerdeman and others 2005; Yao and others 2007; Vega-Lugo and Lim $2008 \mathrm{~b})$. The potential of these fibers have been demonstrated in biomedical applications, such as tissue scaffolds and homeostatic products (Huang and others 2001; Woerdeman and others 2005; Zhang and others 2005). Woerdeman and others demonstrated the ability to electrospin wheat gluten with native and denatured protein at different concentrations, and illustrated the interplay between protein structure and the fluid dynamics of the electrospinning process (Woerdeman and others 2005). They explained that fiber formation by electrospinning is because of the entanglements between the chains and the presence of reversible junctions in the protein, in particular, the breaking and reforming of disulfide bonds that occur via a thiol/disulfide interchange reaction (Woerdeman and others 2005). Also, Li and other coworkers chose collagen and elastin to electrospin the fibers, two of the key structural proteins found in the extracellular matrices of many tissues. These proteins are important modulators for the physical properties of any engineered scaffold, affecting cellular 
attachment, growth and responses to mechanical stimuli (Woerdeman and others 2005). Huang and coworkers were the first to electrospin collagen scaffolds for wound dressing (Huang and others 2001). Shortly thereafter, Matthews and Boland described electrospinning of collagen and elastin fibers for preliminary vascular tissue engineering (Matthews and others 2002; Boland and others 2004).

As a prolamin, zein is relatively more hydrophobic than many other proteins, which is insoluble in water due to the presence of large amount of hydrophobic amino acid residues such as leucine, proline, alanine, and phenylalanine (Gianazza and others 2003). Some experiments of electrospinning zein protein have been carried out to illustrate interaction between protein properties and the characteristics of the electrospining process. Aqueous ethanol has been identified as the solvent of choice for the electrospinning of zein because of its evaporation during the electrospinning process. Yao and coworkers reported that polymer solutions with a zein concentration ranging from 10 to $50 \%(\mathrm{w} / \mathrm{v})$ and $30 \%$ $(\mathrm{v} / \mathrm{v})$ of water in ethanol were electrospun individually in an electric field from 12 to $20 \mathrm{kV}$ to produce fibers (Yao and others 2007). In the electrospinning process, the zein aqueous ethanol solution in the $20 \mathrm{~mL}$ plastic syringe was injected to create a charge imbalance through Taylor cone with a tip diameter of $0.6 \mathrm{~mm}$ at a rate of $6 \mathrm{~mL} / \mathrm{h}$ and placed towards the collection target, approximately $10 \mathrm{~cm}$ from the needle tip. The whole process of electrospinning was carried out at room temperature (Yao and others 2007). 


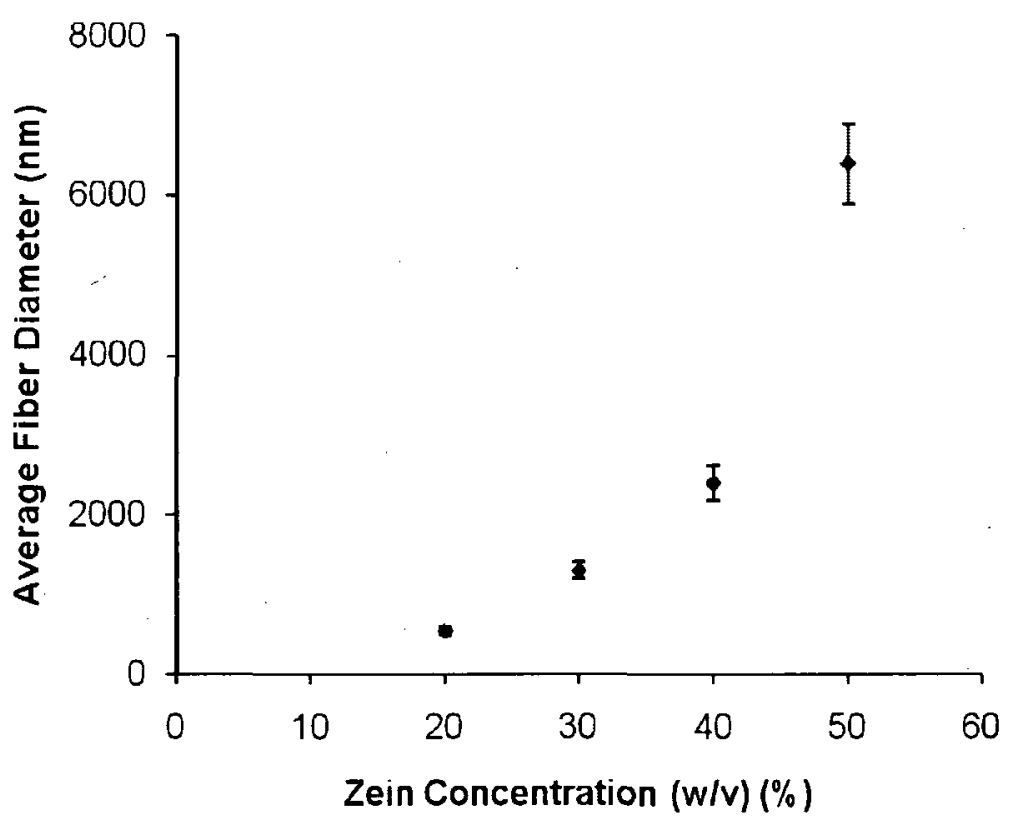

Figure 2 - Average diameters of zein fibers electrospun from aqueous ethanol solutions of different zein concentrations with an ethanol/water ratio of 70:30 (v/V) in an electric field of $2 \mathrm{kV} / \mathrm{cm}$ (Yao and others 2007).

The electrospinning of zein fibers exhibited a concentration dependent on the diameters of the final fibers produced. The average diameters of zein fibers electrospun from 10 to $50 \%(\mathrm{w} / \mathrm{v})$ polymer solution are shown in Figure 2. The results indicate that the fiber diameter dramatically increase with the rise of zein concentration. The increase of zein concentration in aqueous ethanol raises the solution viscosity, which facilitates the formation of fibers. In this study, the most significant factor found to control the diameters of fiber in the electrospinning process is the concentration of zein protein solution.

\section{4 (-)-Epigallocatechin Gallate, EGCG}

Green tea has attracted much attention in recent years, due to its anticancer, antibacterial and anti-aging activity in various biological and pharmaceutical 
studies. The beneficial effects of green tea are attributed to its polyphenol components, namely tea catechins (Wang and others 2008). In the class of polyphenols, catechins belong to the group of flavonoids called flavan-3-ols. Catechins in a polyphenolic group are known to have potential antioxidant activities beneficial to human health in treating vascular, viral, gastrointestinal, microbial and inflammatory diseases medically (Haslam 1989). Currently, catechins have been used to improve food products as a functional ingredient and dietary supplements, as an antimicrobial agent in food products, as chemopreventive and anti-carcinogenic agents, as a nature antioxidant in oils and fats to inhibit lipid oxidation, and as a supplement for animal feeds to improve animal health and products protection (Katiyar and others 2000; Yilmaz 2006). Although green and black teas have a high content of catechin, catechins degrade during the fermentation process of black tea, and its degradation depends on the fermentation temperature and $\mathrm{pH}$ (Obanda and others 2001). The oral bioavailability of catechins is low at less than 5\% (Baba and others 2001; Catterall and others 2003). The half-life of catechins is short because of strong systemic clearance (Cai and others 2002). 


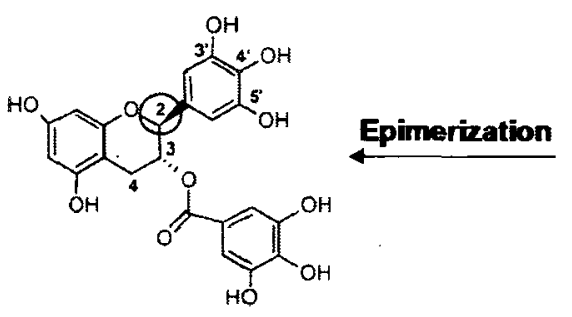

(-)-GCG

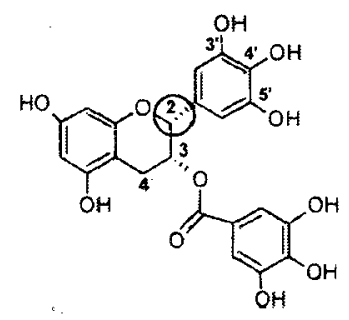

(-)-EGCG

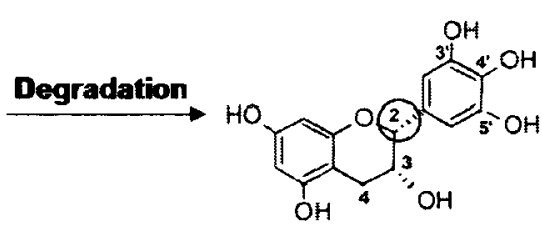

(H)EGC

Figure 3 - Schematic representation for epimerization and degradation of EGCG (Wang and others 2008).

Catechins have been mainly identified as epicatechins, including (-)epigallocatechin (EGC), (-)-epigallocatechin gallate (EGCG) and so on. These compounds can convert to their epimers which are non-epicatechins, i.e. (-)gallocatechin gallate (GCG) and (-)-gallocatechin (GC), respectively (Wang and others 2008). EGCG is the most abundant in green tea, which has been linked with many health benefits. Epidemiologic studies indicate that green tea consumption is associated with cancer-preventative effects. (-)-EGCG is the most abundant and biologically active catechin with respect to anticancer activity in several human cancers (Landis-Piwowar and others 2007). However, (-)EGCG is relatively unstable under neutral or alkaline conditions and could be rapidly degraded, involving deprotonation of hydroxyl groups on the phenol rings, which could be modified through biotransformation reactions (Zhu and others 1997; Chen and others 2001; Landis-Piwowar and others 2007): Moreover, Wang and others showed that the degradation and epimerization occurred simultaneously (Figure 3), and both reactions complied with first-order reaction and their rate constants followed Arrhenius equation, using Matlab programming 
(Wang and others 2008). Based on these references, kinetic studies of EGCG in various aqueous systems should be done.

Therefore, electrospinning fibers as a carrier for catechins, as a micronutrient, would provide an alternative way to potentially resolve these problems. This technology may improve and increase the stability of catechins at high temperatures and $\mathrm{pH}$ values during food processing, food packaging and storage. High-performance liquid chromatography (HPLC) is an important method for testing the concentration of EGCG (Wang and others 2003).

\subsection{Nature of Polyphenol-Protein Interactions}

Protein and polyphenolic compounds interact and combine to form soluble complexes. As reported by Asano and co-workers, peptides and proteins containing proline formed an interaction when combined with polyphenolic compounds, while synthetic polypeptides and proteins lacking proline did not (Asano and others 1982). Polypeptides with a greater percentage of proline tended to form more interaction. Protein molecules which are held together by polyphenolic compounds act as bridges through non-covalent interactions. Asano and coworkers concluded that the complexes are held together by some combination of hydrogen and hydrophobic bonding (Asano and others 1982). Outtrup and coworkers also investigated the involvement of peptides containing proline and found a greater interaction with higher proline content (Outtrup and

others 1987). The interactions between polyphenols and proteins, is helpful for 
forming protein fibers providing good stability for polyphenol both during and after the electrospinning process.

Early research in which pep tides and polyphenols were combined in a model system of a pH 4.02 potassium phosphate buffer suggested that the amount of interaction was related to both protein and polyphenol concentrations (Siebert and others 1996). They set up a model system made up of a polypeptide (gliadin) and a polyphenolic compound (catechin) combined in $0.02 \mathrm{M}(\mathrm{pH} 4.2$ ) phosphate buffer at 25,80 , or $100{ }^{\circ} \mathrm{C}$ for $30 \mathrm{~min}$. Their results showed that as the polyphenol concentration increased at a fixed level of protein, the interaction at first increased; till the interaction reached a plateau, and then declined. The same behavior occurred at fixed polyphenol levels as the protein concentration increase. The concentrations of both protein and polyphenol and their ratios all have strong effects on the amount of interaction produced.

Figure 4 shows that a plateau is reached when either the gliadin or catechin concentration increase while the other is held constant. In the experiment, the peak is reached when the concentrations of gliadin and catechin, are 500 and $750 \mathrm{mg} / \mathrm{L}$ respectively in $0.02 \mathrm{M}(\mathrm{pH} 4.2)$ potassium phosphate buffer and heated for 30 min at $80^{\circ} \mathrm{C}$ (Siebert and others 1996). In the other word, the amount of interaction formed depends on the concentrations of protein and polyphenol and on their ratio. The greatest interaction is produced when the number of polyphenolic bonds equals the number of protein binding sites. A model in which interaction-active proteins have a fixed number of polyphenol binding sites at a given temperature accounts for this behavior (Siebert and others 1996). 


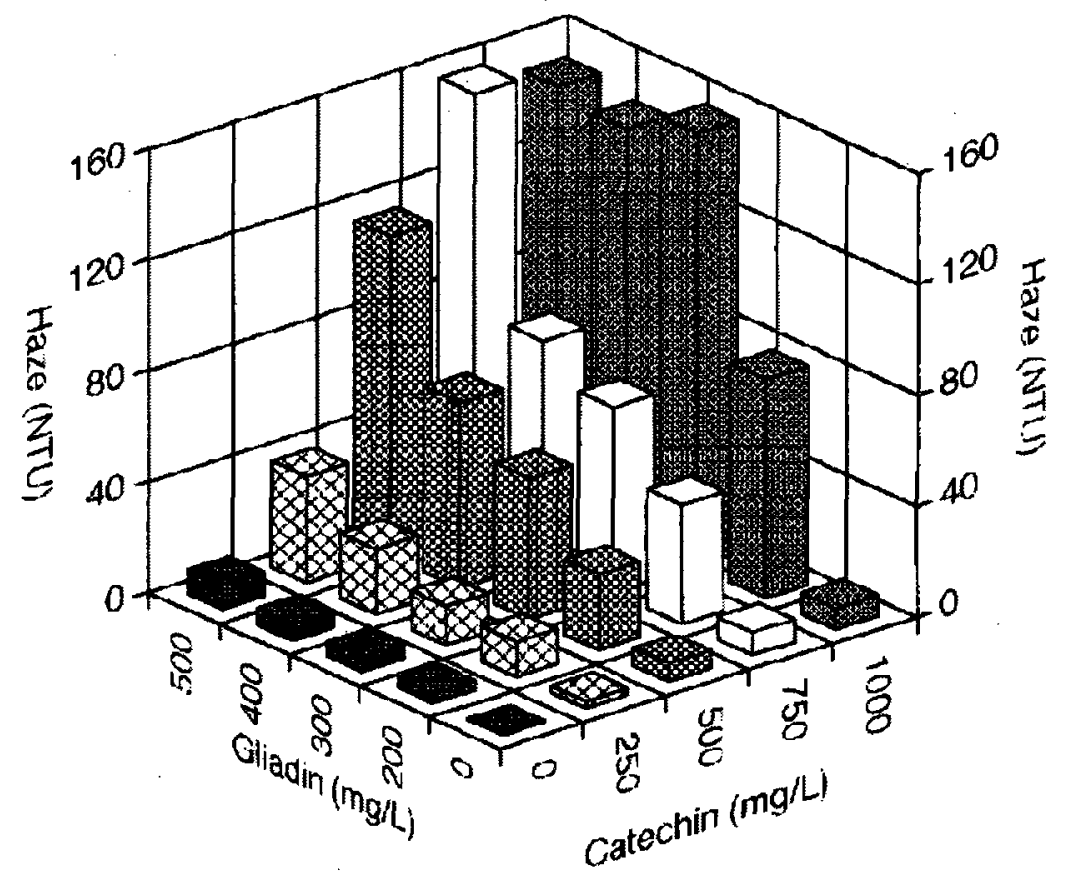

Figure 4 - Interaction produced when various concentrations of gliadin and catechin were combined in $0.02 \mathrm{M}(\mathrm{pH}$ 4.2) potassium phosphate buffer and heated for $30 \mathrm{~min}$ at $80^{\circ} \mathrm{C}$ (Siebert and others 1996).

Temperature is the one of the most important factors in the interaction between protein and polyphenol. The greater amount of interaction observed upon heating protein at higher temperatures suggest that hydrophobic binding is important in the interaction between proteins and polyphenols. The higher temperatures used may contribute more hydrophobic sites either kinetically or possibly by breaking hydrogen bonds and unraveling the protein structure to a greater extent. Since hydrophobic groups tend to be concentrated in the interior of water-soluble proteins and one of the more likely mechanisms for the proteinpolyphenol interaction is hydrophobic bonding, it is likely that more hydrophobic (polyphenol binding) sites could be exposed by heating (Siebert and others 1996). 


\section{JUSTIFICATION AND OBJECTIVES}

Although electrospinning technology has been applied in various areas such as medicine and textiles, the use of electrospinning for protecting micronutrients in food process applications has not been reported in the literature. However, it is obvious that electrospinning is a promising technique for forming fibers. Recently, several studies have shown that protein can be spun into fibers, including silk fibroin, collagen, gelatin, wheat gluten, and zein, using the electrospinning technique (Jin and others 2002; Miyoshi and others 2005; Buttafoco and others 2006). This technique provides an opportunity of spinning zein protein fiber as a carrier for stabilizing EGCG.

In this study, we hypothesize the electrospun zein fibers can be used as an effective carrier to stabilize EGCG. The objectives of this project are as follows:

- Optimize the electrospinning process setup and conditions for spinning zein protein fibers;

- Investigate the interaction between protein structure and physicochemical characteristics of electrospun fiber;

- Study the effects of EGCG on the electrospun zein fibers and determine the efficacy of zein fibers for stabilizing EGCG;

- Study the degradation kinetics of EGCG in aqueous model systems;

- Develop and validate kinetic models for EGCG degradation reactions. 


\section{ELECTROSPINNING OF ZEIN PROTEIN FIBERS}

\subsection{Introduction}

Recently, electrospinning has received a great deal of attention as a convenient method for producing fibers with submicron in diameters. The electrospinning process is an efficient, practical and rapid method for producing the fibers ranging in size from microns down to a nanometer or less. Matthews and other coworkers have successfully produced tissue-engineering scaffolds composed of collagen fibers (Matthews and others 2002). A variety of electrospinning experiments have been carried out with different kinds of protein such as wheat protein, zein protein and soy protein, at different solution concentrations to illustrate the interplay between protein structure and the characteristics of the electrospinning process (Yao and others 2007).

Zein is a class of prolamin from corn, and is an abundant protein found in corn gluten meal, a coproduct of corn wet milling (Selling and Sessa 2007). Zein possesses an additional benefit of being renewable and biodegradable and has also been used in the pharmaceutical industry to coat capsules, provide protection, control release of active ingredients and mask flavors and aromas (Shukla and Munir 2001). Also, zein microspheres have been used as carriers to protect drugs from stomach acid (Yao and others 2007). Because zein is hydrophobic, it has been used in the food industry as a coating material for candies, rice, dried fruits and nuts. Zein has also been investigated for use as a structural material in packaging applications because of its film forming properties of film formation. In particular, zein forms tough and glossy films or 
coatings that are resistant to microbial attack. A zein coating, therefore, can function as an oxygen, lipid and moisture barrier. Zein has also been investigated for use as a fiber (Miyoshi and others 2005). These findings suggest that zein is a promising biomaterial that can be applied in various areas.

In this research, due to the high ratio of nonpolar amino acid residues and lack of basic amino acids, prolamin proteins, such as corn zein, are soluble in aqueous alcohol, an appropriate solvent for electrospinning. Using alcohol as a solvent for electrospinning is important because as a solvent it can vaporize readily from the fiber as the polymer jet takes flight in the air, forming dry and solid fibers upon loading on the collection target. Also ethanol is considered to be a solvent for food applications.

The scanning electron microscopy (SEM) produces a vivid and seemingly three-dimensional picture of a specimen surface over a wide range of magnification (Hayat 1978). It routinely operates between the magnification range of a stereo light microscope up to 100,000 fold, with a resolution limit of 2.5 $\mathrm{nm}$ (Hoppert 2003). The SEM uses a focused electron beam to scan across the specimen surface. The diameter of the focused beam on the specimen surface determines the resolution of the image. The structure of the sample must be stable after exposure to the electron beam and also to the vacumm conditions. In order to obtain good images of most non-conductive specimens, the sample must first be covered with a thin metal coating. This increases conductivity and the secondary and back-scattered electron emission, and also reduces thermal 
damage (Hoppert 2003). SEM has been extrensively to study the microstructure of biological material (Hayat 1978).

In this study, the electrospinning method was used to produce zein protein fibers. Scanning electron microscopy has been used to determine the morphology of the electrospun fibers. The main focus was to determine the factors that influenced the morphology and diameter of the electrospun fibers.

\subsection{Materials}

Zein was purchased from Sigma-Aldrich (St. Louis, Mo., U.S.A.) and was used as received, without further purification. Ethanol of $100 \%$ purity was supplied by Commercial Alcohols (Brampton, Ont., Canada). Fiber-forming solutions were prepared by dissolving zein powder (10 to $30 \% \mathrm{w} / \mathrm{w})$ in various aqueous ethanol solutions (60 to $90 \% \mathrm{w} / \mathrm{w}$ ethanol) at room temperature under magnetic stirring until the protein completely dissolved.

\subsection{Methods}

Zein solutions were electrospun at a $0.3 \mathrm{~mL} / \mathrm{h}$ feed rate using a 20-gauge blunt end stainless steel spinneret which was attached to the positive electrode of a direct current (DC) power supply (Ormond Beach, Fla., U.S.A.). The spinneret was connected through Teflon tubing to a $3 \mathrm{~mL}$ plastic syringe containing the zein solution. The solution was fed to the spinneret by means of an infusion pump (Figure 1). When the power supply was set above the critical voltage of $\sim 12 \mathrm{kV}$, the charge build-up in the pendant droplet at the spinneret tip 
caused the zein solution to eject towards the grounded collector which was positioned at $10 \mathrm{~cm}$ from the spinneret tip. As the polymer jet took flight in the air, the solvent evaporated rapidly, forming solidified fibers on the collector. To prevent the spinneret tip from blocking due to evaporation of solvent, a holder containing ethanol was attached around the tip of the spinneret. This provided a blanket of ethanol vapor around the Taylor cone structure, thereby enabling continuous fiber spinning. The fibers were collected on an aluminum foil attached on the surface of the collector. All electrospinning experiments were carried out at $21 \pm 2{ }^{\circ} \mathrm{C}$.

\section{Morphology of electrospun fibers}

The morphology of the electrospun fibers was examined using a scanning electron microscope (SEM) (Hitachi S-570, Japan) after sputter-coating the samples with gold under vacuum. All SEM evaluations were conducted at an accelerating voltage of $10 \mathrm{kV}$. Diameters of the electrospun fibers were measured using Image-Pro Plus 6.1 software (Media Cybernetics, Bethesda; Md., U.S.A.). For fiber diameter determination, 100 measurements were taken from each micrograph.

\subsection{Results and Discussions}

Previous study have been reported that the diameter and morphology of electrospun zein fibers were strongly affected by a number of processing parameters, such as polymer concentration, aqueous ethanol concentration, applied DC voltage, the distance between spinneret and collector, and the 
solution flow rate (Miyoshi and others 2005; Yao and others 2007; Torres-Giner and others 2008). Similar to those studies, the electrospun fibers from the present study mainly adopted ribbon-like structures with morphologies and diameters that were strongly affected by protein concentrations and aqueous ethanol concentrations; however, within the range of voltage tested, the change in electric field strength did not result in significant modifications of fiber morphology.

\subsubsection{Effects of zein protein concentration}

Figure 5 shows the scanning microscopy images of zein polymer electrospun at $15 \mathrm{kV}$ in $70 \%$ ethanol at various zein concentrations (10 - 30\% $w / w)$. As the concentration of zein protein solution increased, the diameters of nanofibers increased. Moreover, the morphology changes from beads to ribbonlike nanofibers. At the lowest zein concentration tested $(10 \%, w / w)$, electrospraying occurred, producing droplets instead of fibers. Unlike other dilute polymer solutions reported elsewhere which exhibit round to oval solid spheres (Zong and others 2002; Jun and others 2003; Huang and others 2004; Wang and others 2005), the droplets we observed here appeared to possess an outer skin which imploded inwards as the solvent evaporated (Figure 5A). This observation provides indirect evidence that the ribbon morphology may be caused by the formation of "garden hose" structures which collapse to form ribbons as they land on the collector target. This hypothesis seems to agree well with the observations by Woerdeman and others (2005) on their cross-section micrographs of ribbon-

shaped electrospun wheat gluten fibers spun using 1,1,1,3,3,3-hexafluoro-2- 
proponol as the solvent. The electrospinning of zein fibers exhibited a concentration dependent on the diameters of the final fibers produced (Yao and others 2007$)$. At $20 \%(\mathrm{w} / \mathrm{w})$ polymer concentration and higher, continuous fibers were observed, with concomitant increases in diameter and thickness as polymer concentration increased (Figure 5B and $5 \mathrm{C}$ ). The formation of continuous fibers at higher polymer concentrations can be attributed to the increased molecular chain entanglement, which prevented the polymer jet from breaking up into droplets. The increased polymer concentration also led to an increased interaction between the polymer and solvent, thereby reducing the propensity of the polymer solution to form droplets under the influence of surface tension of the solvent.
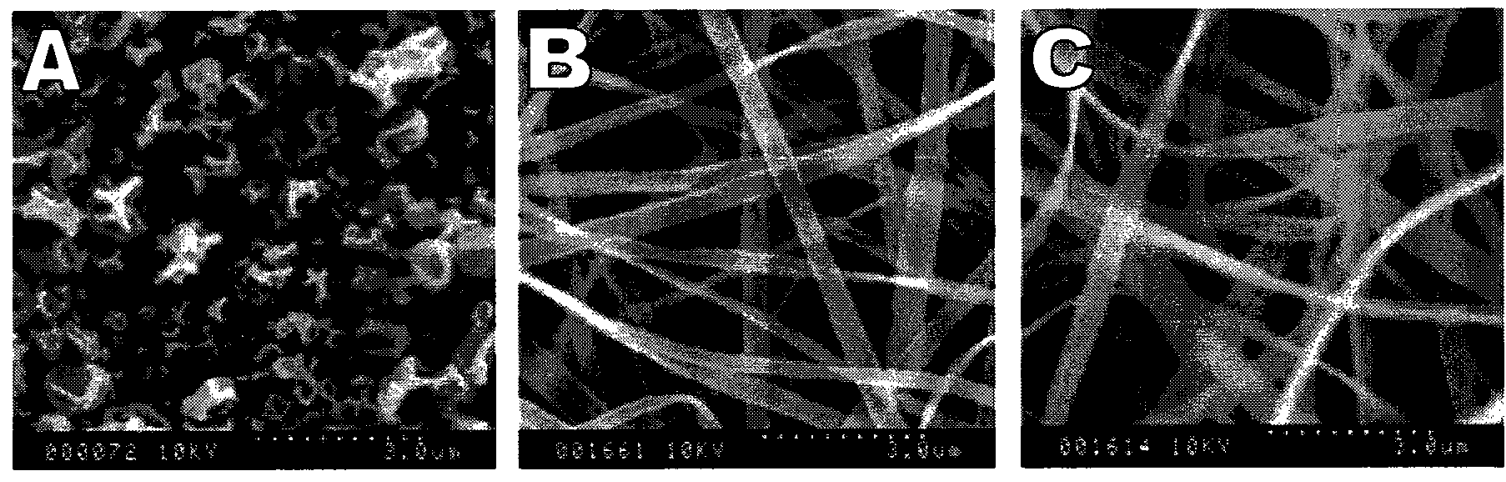

Figure 5 - SEM images of zein fibers electrospun at $15 \mathrm{kV}$ in $70 \%$ ethanol at various zein concentrations. A: $10 \%$ zein (276 $\pm 26 \mathrm{~nm}$ ); B: $20 \%$ zein (386 \pm 32 $n m)$; C: $30 \%$ zein $(462 \pm 43 \mathrm{~nm})$.

The results from Figure 6 indicated that the fiber diameter was dramatically increased with an increase in zein concentration. At higher zein concentration, the average diameter was thicker than that at lower concentration. In this 
research, solution concentration was showed to be one of the most significant factors controlling the fiber diameter in the electrospinning process.

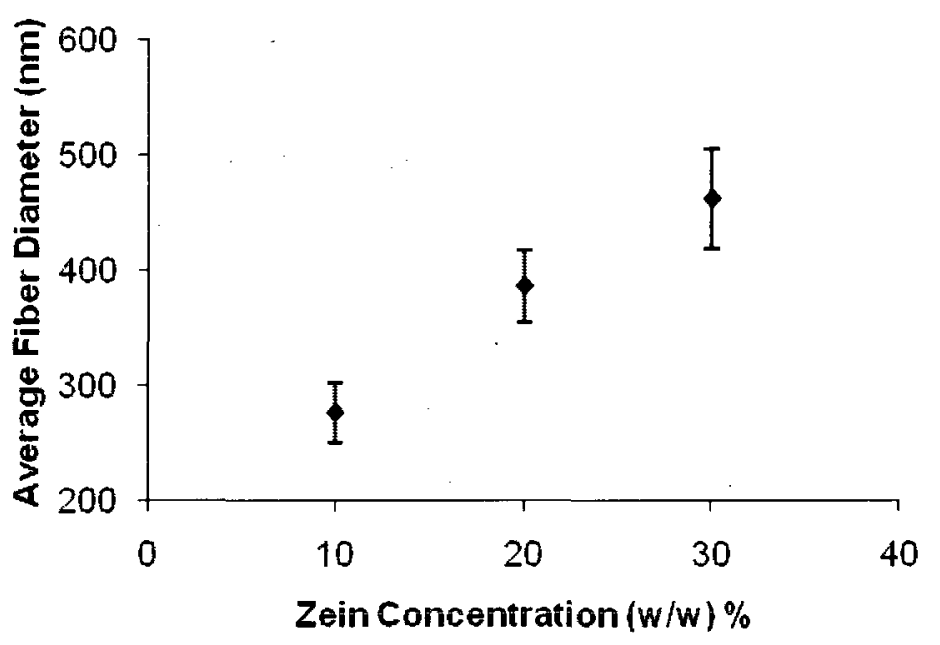

Figure 6 - Average diameters of zein fibers electrospun from different zein concentrations dissolved in $70 \%$ aqueous ethanol (W/W) under an electric field of $15 \mathrm{kV}$.

\subsubsection{Effects of aqueous ethanol concentration}

Figure 7 shows SEM images of fibers electrospun at $15 \mathrm{kV}$ from $20 \%$ zein dissolved in various aqueous ethanol concentrations (60 to $90 \%$ ). As the concentration of aqueous ethanol concentration increased, the diameters of fibers increased, from $369 \pm 32 \mathrm{~nm}$ to $683 \pm 62 \mathrm{~nm}$ (Figure 8). Fiber diameters tended to become smaller when a lower ethanol concentration $(60 \%)$ was used (Figure $7 \mathrm{~A})$. A higher concentration of ethanol $(90 \%)$ resulted in the formation of larger fibers with less consistent morphology (Figure 7D). Increasing the ethanol concentration should lower the volume charge density of the polymer jet (Theron 
and others 2004), thereby inducing greater whipping instability and producing smaller fibers. However, this effect was counteracted by the increased volatility of the solvent and the greater tendency for the polymer solution to solidify and prevent fiber drawing. Based on these findings, ethanol concentrations of 60 to $70 \%$ were judged to be the optimal for electrospinning of $20 \%(w / w)$ zein solutions.
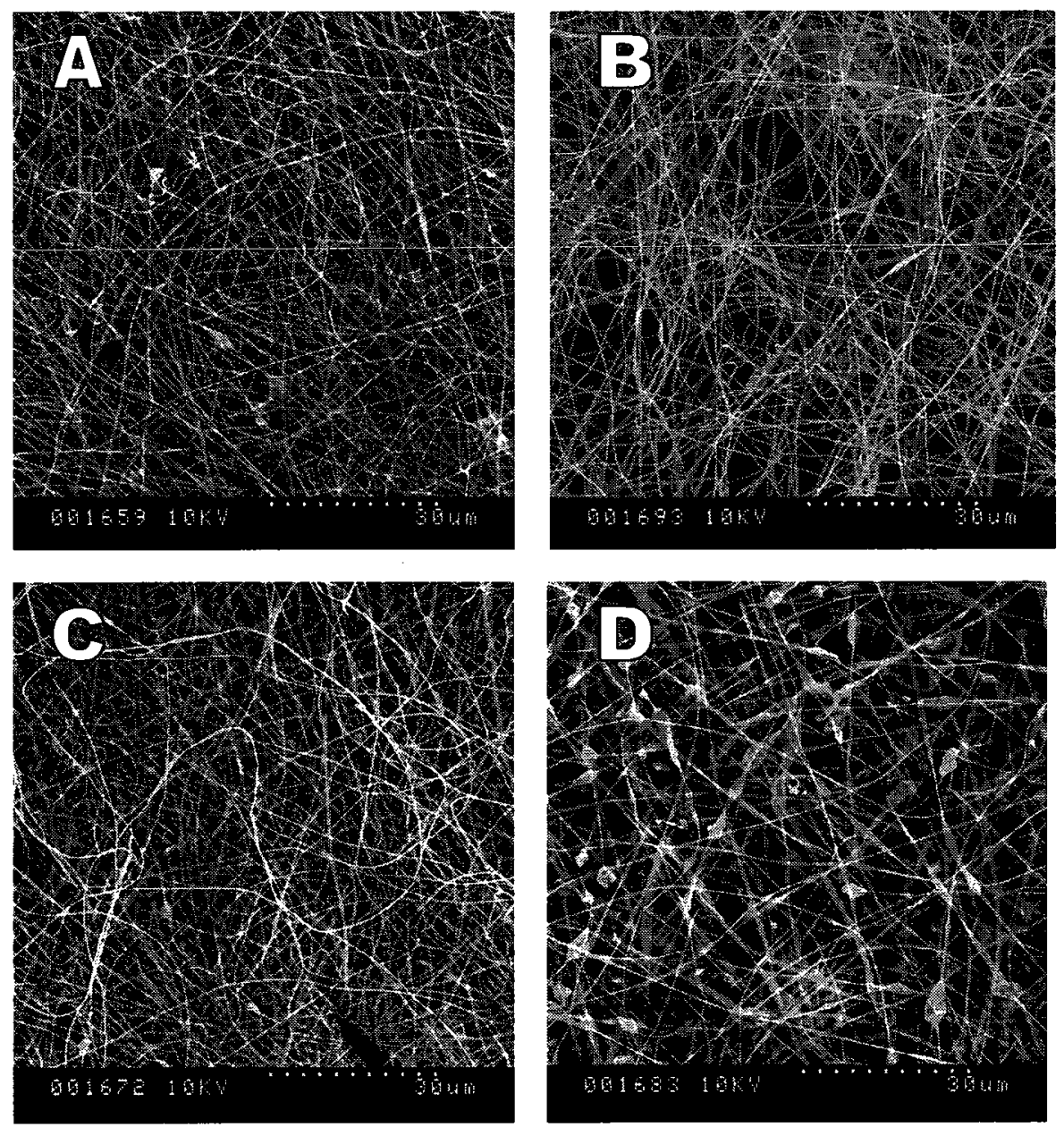

Figure 7 - SEM images of zein fibers electrospun at $15 \mathrm{kV}$ at $20 \%$ zein dissolved in various ethanol concentrations. A: $60 \%$ ethanol ( $369 \pm 32 \mathrm{~nm}$ ); B: $70 \%$ ethanol (402 $\pm 37 \mathrm{~nm})$; C: $80 \%$ ethanol $(518 \pm 47 \mathrm{~nm}) ;$ D: $90 \%$ ethanol $(683 \pm 62 \mathrm{~nm})$. 


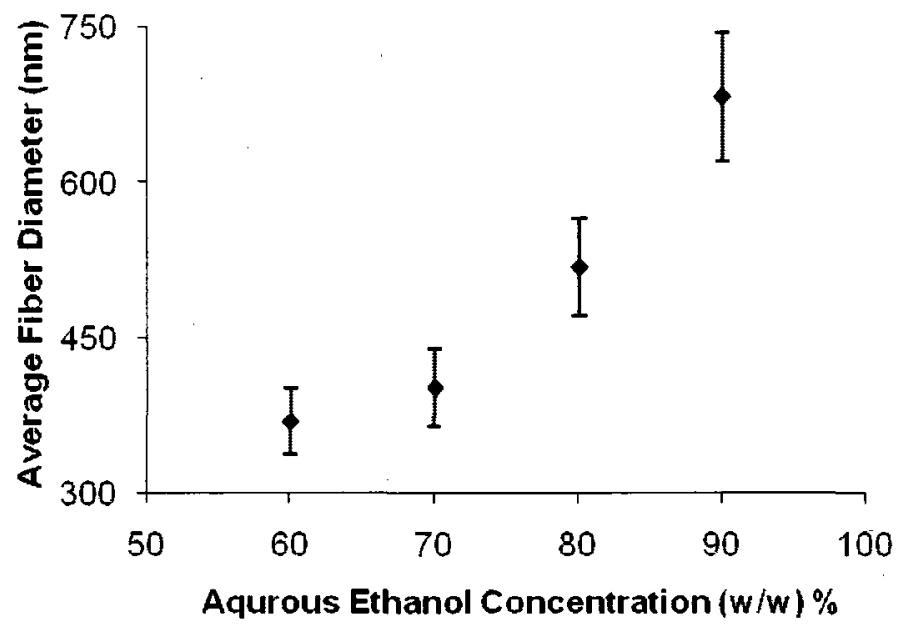

Figure 8 - Average diameters of zein fibers electrospun from polymer solutions of different ethanol/ water ratios of 60:40; 70:30; 80:20; $90: 10(w / w)$ under an electric field of $15 \mathrm{kV}$.

\subsubsection{Effects of applied electric field}

According to the references, there is another significant factor impacting the fiber diameter in the electrospinning process (Miyoshi and others 2005; Yao and others 2007; Torres-Giner and others 2008). In contrast, within the range of voltage tested (15 and $20 \mathrm{kV}$ ), as the electric field decreased, the diameter of fibers became slightly smaller. Overall, the change in electric field strength did not result in significant modifications of fiber morphology (Figure 9). 

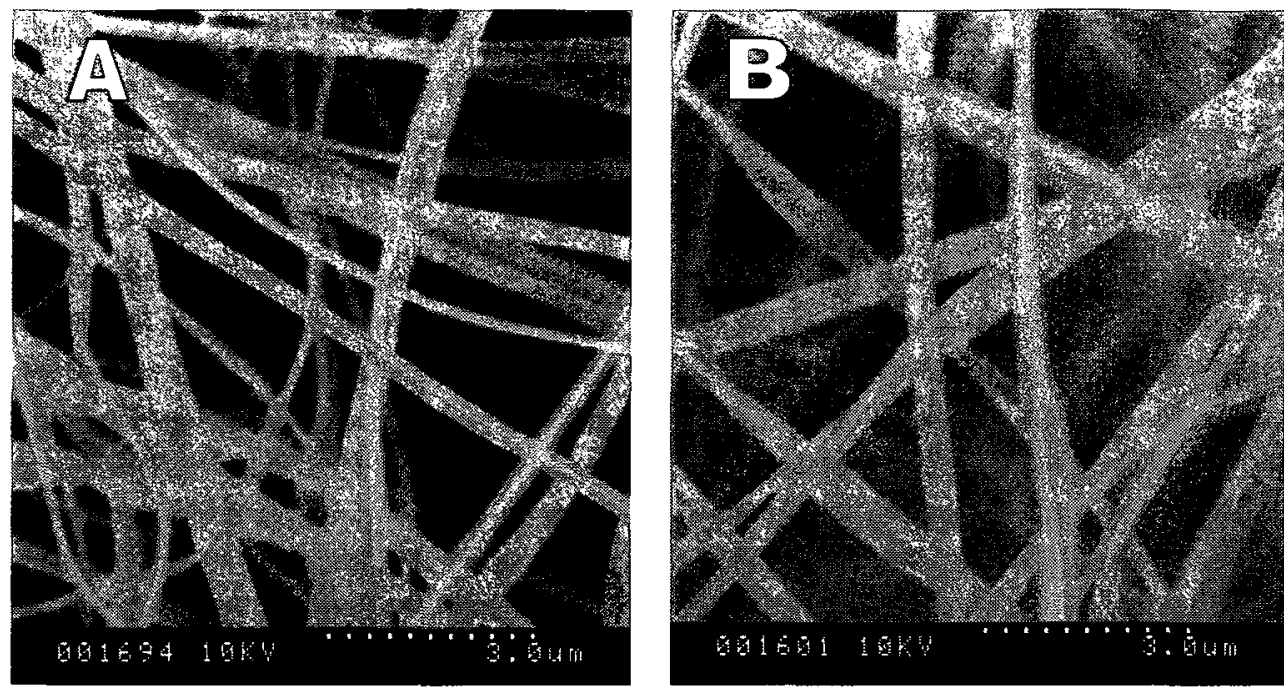

Figure 9 - SEM images of 20\% zein polymer dissolved in $70 \%$ ethanol concentration at various voltages. A: $15 \mathrm{kV}(347 \pm 27 \mathrm{~nm}) ; \boldsymbol{B}: 20 \mathrm{kV}(311 \pm 23$ $n m)$.

\subsection{Conclusions}

In this study, an inverted spinneret setup was adopted for continuous electrospinning of zein fiber. The morphology of the resulting fibers was mainly affected by the aqueous ethanol concentration, zein protein concentration, and to a lesser extent, the electric field strength. The optimal condition for forming beadless fibers was found to be $20 \%$ protein, $70 \%$ alcohol, and $15 \mathrm{kV}$. 


\section{EFFECTS OF EGCG ON ZEIN FIBERS}

\subsection{Introduction}

Previously, zein protein has been successfully and continuously electrospun into protein fibers by a developed method under various conditions. Overall, the optimal condition for forming bead-less fibers was found to be $20 \%$ protein, $70 \%$ alcohol, and $15 \mathrm{kV}$ for the further study.

As explained, proteins have been known to interact strongly with polyphenol through hydrogen bonding and hydrophobic interactions. Madhan and others (2005) reported that although there were no major changes in the secondary structure of collagen after treatment with catechin, the treated collagen resisted solubilization in urea and shrinkage upon exposure to elevated temperatures. They suggested that hydrogen bonding and hydrophobic interactions are the driving forces contributing to the stabilization of collagen by catechin. Siebert and others (1996) showed that heating gliadin samples in the presence of polyphenol led to an increase in haze, and speculated that hydrophobic interactions played a more important role than hydrogen bonding. In the study involving the interaction between EGCG and human serum albumin, Maiti and others (2006) postulated that hydrophobic interaction is more prominent during initial association, while van der Waals interactions and hydrogen bonding are important for stabilizing the complex. EGCG is not stable under alkaline conditions and may undergo oxidation, polymerization and epimerization during thermal processing (Zhu and others 1997; Sang and others 2005; Wang and others 2006; Yusuf 2006). In view of the propensity of protein to interact with polyphenols, we hypothesize that 
encapsulating EGCG in zein electrospun fiber may result in enhanced EGCG stability.

Using EGCG as a model bioactive compound, the objectives of this study were to determine the effects of EGCG on protein fibers and to evaluate the stability of the encapsulated EGCG in zein fibers in an aqueous model.

\subsection{Materials}

Zein was purchased from Sigma-Aldrich (St. Louis, Mo., U.S.A.) and was used as received, without further purification. Ethanol of $100 \%$ purity was supplied by Commercial Alcohols (Brampton, Ont., Canada). Purified EGCG powder, extracted from green tea, was donated by Mitsui Norin Co., Ltd. (Fujieda, Japan). Acetic acid and acetonitrile were purchased from Fisher Scientific (Ottawa, Ont., Canada). Fiber-forming solution containing 20\% (w/w) EGCG was prepared by first dissolving EGCG in $70 \%(w / w)$ aqueous ethanol, followed by dispersing zein powder to give $20 \%(w / w)$ zein solution.

\subsection{Methods}

\subsubsection{Electrospinning}

EGCG-containing solution was electrospun as described in section 4.3.1.

\subsubsection{Morphology of electrospun fibers}

Freshly spun fibers were examined using a scanning electron microscope (SEM) as described in section 4.3.2. 


\subsubsection{HPLC analysis}

Analysis of EGCG was performed in a HPLC (2695/2696 Waters, Milford, Mass., U.S.A.) equipped with a photodiode array detector and a C18 reversed phase column ( $150 \mathrm{~mm} \times 3.9 \mathrm{~m} / 4 \mu \mathrm{m}$, Waters). Mobile phases consisted of $2 \%$ (v/v) acetic acid in water (eluent A) and $100 \%$ acetonitrile (eluent B), respectively. The isocratic system was run with $88 \%$ eluent $A$ and $12 \%$ eluent $B$. The sample injection volume was $20 \mu \mathrm{L}$. The flow rate was at $0.8 \mathrm{~mL} / \mathrm{min}$ and EGCG was detected at $275 \mathrm{~nm}$ after an elution time by $10 \mathrm{~min}$.

The standard EGCG was stored at $-30^{\circ} \mathrm{C}$ in the freezer. The calibration curve (Figure 33) was constructed by diluting EGCG solution to yield the final concentration of $0.02,0.04,0.06,0.08$ and $0.10 \mathrm{mg} / \mathrm{mL}$, which are based on the pre-calculated concentration of EGCG encapsulated in the fibers. Calibration curves were linear with the coefficients of determination $\left(R^{2}\right)$ close to unity 0.9994 for EGCG. Based on the EGCG standard calibration, the concentration of EGCG $(c)$ encapsulated within the electrospun fibers was determined. The amount of EGCG encapsulated in fibers ( $\left.W_{E G C G i}\right)$ was calculated as followed:

$$
W_{E G C G_{i}}=c \times L
$$

where $L$ is the total volume of ethanol and water for dissolving zein fibers and precipitating zein protein, respectively. The initial amount of EGCG present in the fibers ( $\left.W_{E G C G_{o}}\right)$ was calculated by multiplying the weight of electrospun fibers $\left(W_{f}\right)$ with the percentage of EGCG containing in the fibers, described as followed: 


$$
W_{E G C G_{o}}=W_{f} \times\left(\frac{W_{E G C G_{o}}{ }^{\prime}}{W_{Z e i n}+W_{E G C G_{o}}{ }^{\prime}} \times 100 \%\right)
$$

The percentage of residual EGCG $(p)$ was calculated as the ratio of $W_{E G C G i}$ to $W_{E G C G o}$ in the following formulation,

$$
p \%=\frac{W_{E G C G_{i}}}{W_{E G C G_{o}}} \times 100 \%
$$

\subsubsection{EGCG extraction and determination}

To determine if zein fibers were capable of immobilizing and protecting EGCG in an aqueous environment, about $0.8 \mathrm{mg}$ of EGCG-containing fibers (supported on the aluminum foil) were submerged in water $\left(4 \mathrm{~mL} ; 20 \pm 2{ }^{\circ} \mathrm{C}\right.$ ) in separate beakers to elute the "extractable" EGCG from the fibers (Figure 10): At 20,40 and $60 \mathrm{~min}$, water was decanted from each beaker and followed with $70 \%$ aqueous alcohol $(10 \mathrm{~mL})$ to dissolve the fibers. The zein protein was precipitated from the solution by adding water $(5 \mathrm{~mL})$ gradually over $60 \mathrm{~min}$ duration. The resulting suspension was centrifuged at $4,000 \times \mathrm{g}$ for $5 \mathrm{~min}$ to settle the protein. The supernatant was then filtered through a $0.45 \mu \mathrm{m}$ nylon filter and analyzed by HPLC. 


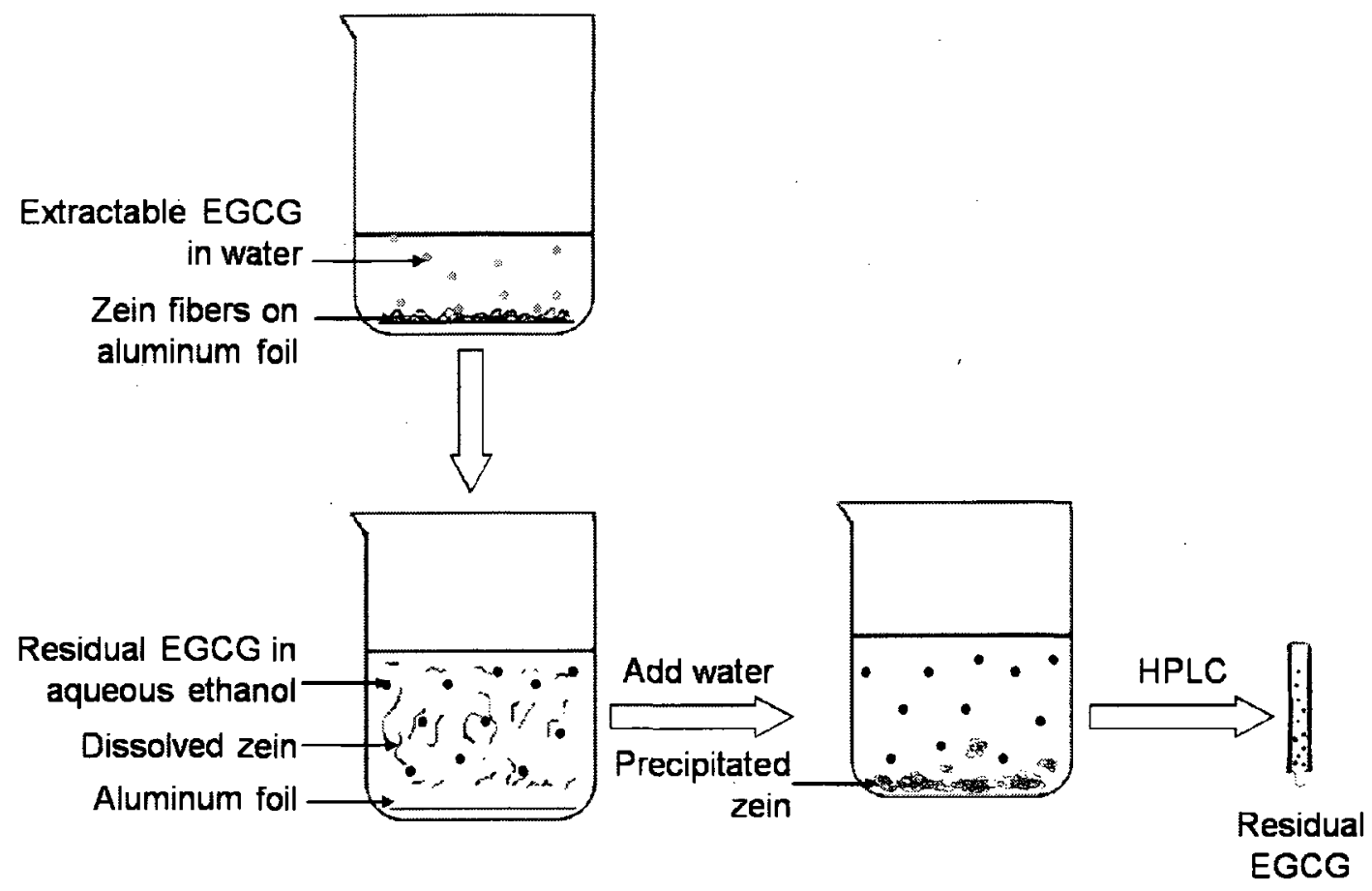

Figure 10 - Schematic representation of EGCG extraction process.

To study the aging and relative humidity (RH) effected on the stability of EGCG, the fibrous mats were held at $21{ }^{\circ} \mathrm{C}$ immediately after spinning, within hermetically sealed glass jars containing either desiccant or saturated $\mathrm{NaCl}$ solution to provide 0 and $75 \% \mathrm{RH}$ conditions, respectively (Figure 11). Samples were retrieved from the glass jars and tested after 1,2 and 3 days to determine EGCG concentrations as described above. The freshly spun fibers (day 0 ) were also tested and served as controls. Residual EGCG remaining was calculated as the ratio of the encapsulated EGCG in fibers divided by the calculated EGCG based on the amount added in the formulation (Equation 5.3). 


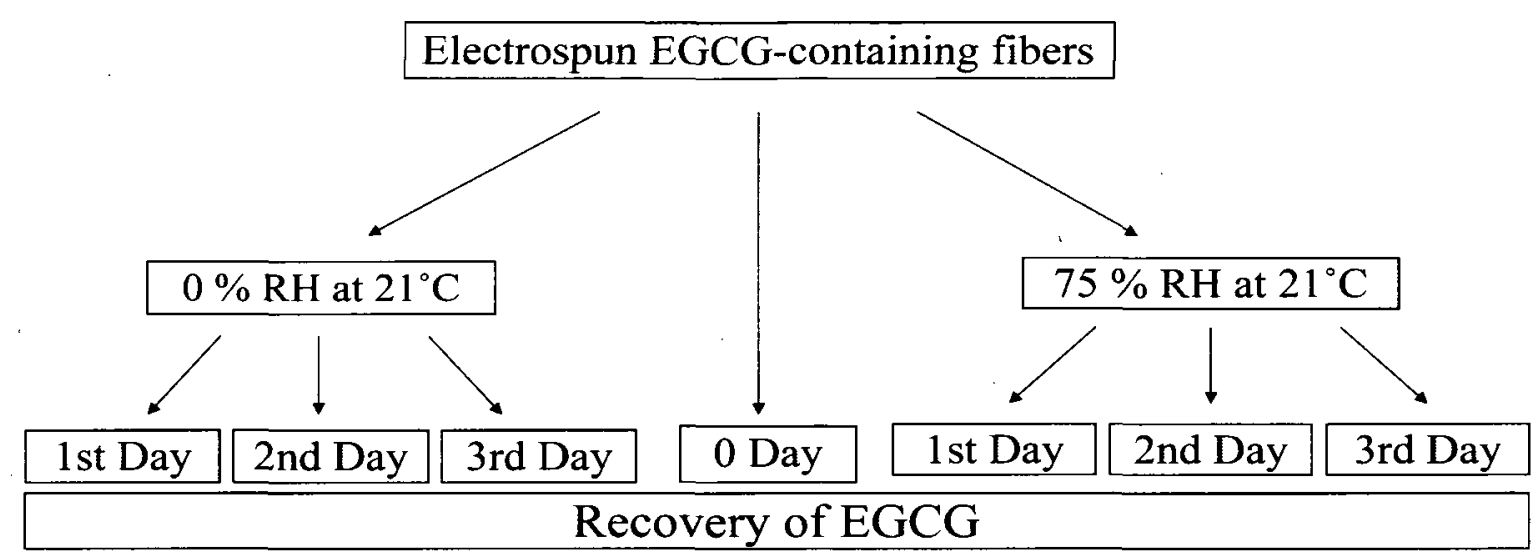

Figure 11 - Schematic representation of effects on EGCG aging and humidity process.

\subsubsection{Fourier transform infrared (FTIR) evaluation}

Infrared analysis of electrospun fibers was conducted using an IRPrestige21 FTIR spectrometer (Shimadzu Corporation, Kyoto, Japan) equipped with a total attenuated reflection accessory (Pike Technologies, Madison, Wis., U.S.A.). Prior to testing each sample, a measurement was taken of air as the background. IR spectra were collected at room temperature for zein fibers with and without EGCG using 30 averaged scans at $4 \mathrm{~cm}^{-1}$ resolution. Curve fitting was performed using Grams/32 spectral analysis software (Galactic Industries Corporation, Salem, N.H., U.S.A.). The Gaussian method was used to fit spectra for zein fibers (with and without EGCG) in the region of 1780 to $1480 \mathrm{~cm}^{-1}$. The bands for EGCG were fitted using the Lorentzian function because of their sharper peak shape. The positions of the underlying bands were determined from the secondderivative of the respective spectrum. 


\subsubsection{Statistical Analysis}

All the reactions were performed in at least triplicate, and the average data were presented as means \pm standard deviations. An analysis of variance single factor test was used to examine the significance at the $\mathrm{P}<0.05$ confidence level.

\subsection{Results and Discussion}

\subsubsection{Effects of EGCG on morphology of zein fibers}

Electrospun zein fibers with and without EGCG had similar ribbon-like morphologies, although EGCG-containing fibers tended to be larger in width, which was caused by the additional EGCG incorporated (Figure 12A and 12B). We observed that the fibrous mats congregated when they were immersed in water, likely due to zein hydrophobicity towards the surrounding water. Electrospun zein fibers with and without EGCG resisted the solubilization after 30 min immersion in water. However, the EGCG-containing fibers became larger in diameter after water immersion probably because of the increased swelling due to the interaction of EGCG with water (Figure 12C and 12D). 

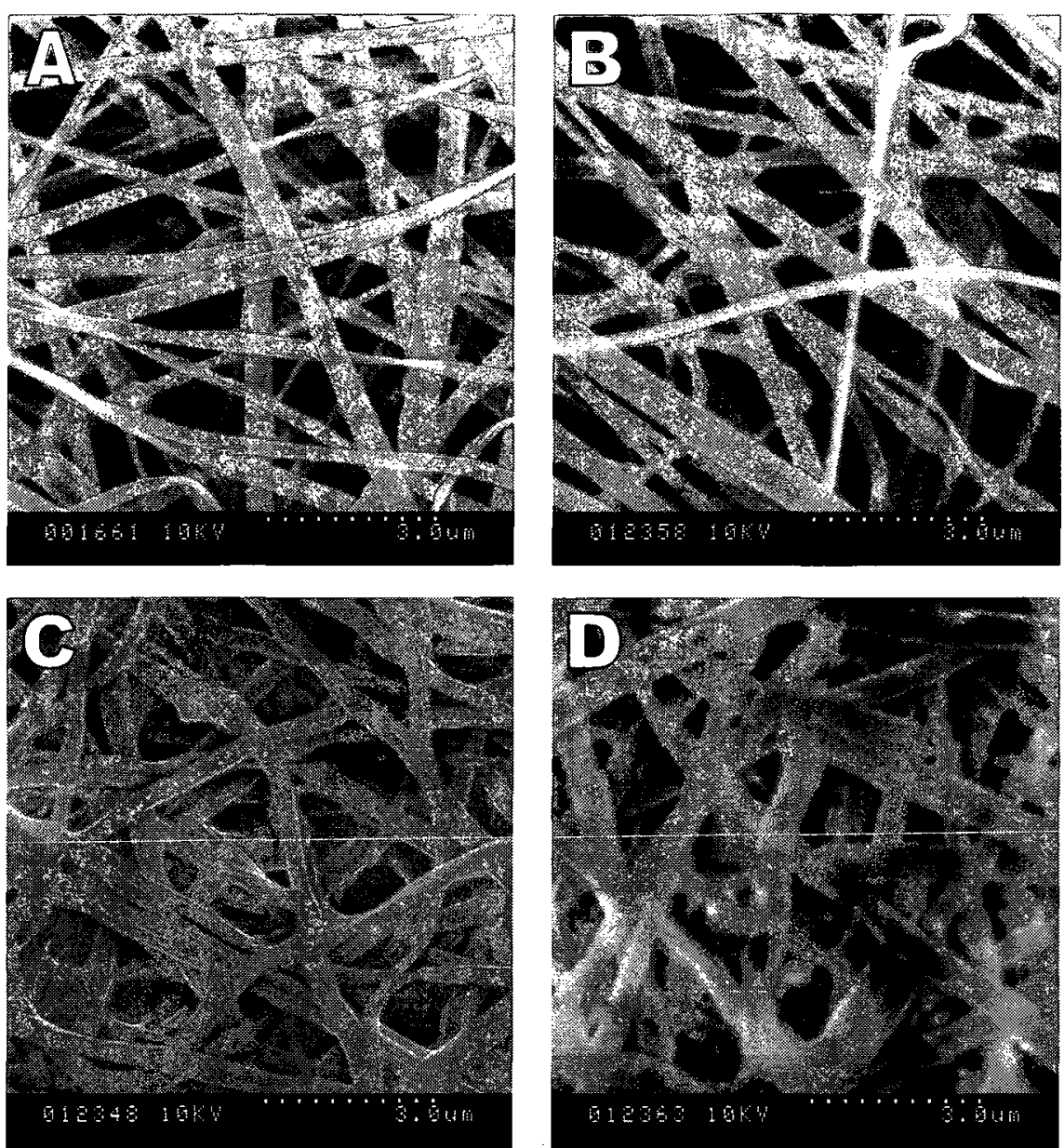

Figure 12 - SEM images of electrospun fibers (20\% zein; $70 \%$ w/w ethanol) before and after immersion in water for $30 \mathrm{~min}$. A: zein fibers before water immersion (386 $\pm 32 \mathrm{~nm}$ ); B: zein + EGCG before water immersion (472 \pm 46 $n m) ;$ C: zein fibers after water immersion ( $457 \pm 38 \mathrm{~nm}$ ); D: zein + EGCG after water immersion (631 $\pm 56 \mathrm{~nm})$.

\subsubsection{Previous stability study of EGCG in zein fibers}

The effects of aging time and relative humidity $(\mathrm{RH})$ on percent residual EGCG remaining are summarized in Figure 13. The plots level off with increasing extraction time, indicating that the majority of water-extractable EGCG eluted in about $60 \mathrm{~min}$. Submerging fibers in water immediately after electrospinning (day 
0 , without aging) resulted in about $82 \%$ remaining, implying that a considerable amount of EGCG was lost in the water. In contrast, fibers aged for 1, 2 and 3 days at $0 \% \mathrm{RH}$ exhibited almost complete $(\sim 98 \%)$ residual EGCG remaining percentage. Furthermore, within the 3-day aging period, little difference in percent residual EGCG was observed. This suggested that interaction between zein and EGCG continued to evolve after the fibers were spun, possibly due to the secondary structural changes of zein that facilitated the interaction between EGCG and zein.

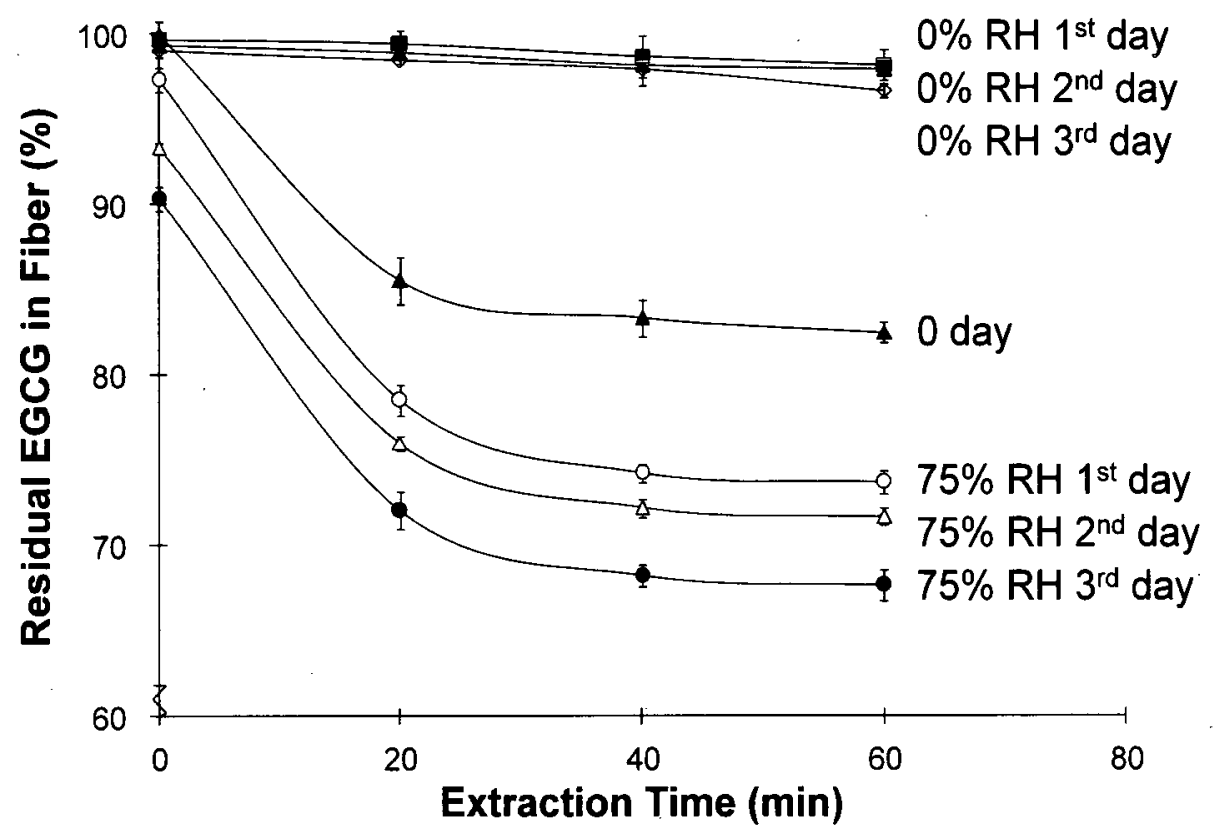

Figure 13 - Effects of aging, relative humidity, and extraction time on percent residual EGCG from electrospun zein fibers after immersing in water for up to 60 $\min$.

In contrast, when the fibers were aged at $75 \% \mathrm{RH}$, much lower EGCG recoveries $(66-73 \%)$ were obtained. Samples tested at time 0 (not subjected to $\mathrm{RH}$ treatment) exhibited lower total EGCG as aging time increased, indicating 
that the EGCG might have been progressively degraded with aging time at the elevated $\mathrm{RH}$ condition. At $0 \% \mathrm{RH}$, hydrogen and hydrophobic interactions of the aromatic rings of EGCG molecule with the hydrophobic residues of zein, may have developed which effectively resulted in the EGCG not being extractable in water during subsequent immersion (Hammel and others 2004; Madhan and others 2005). At $75 \% \mathrm{RH}$, the presence of moisture appeared to hamper these hydrophobic interactions from establishing, resulting in a greater EGCG loss in water, although substantial amounts of EGCG (66 to $73 \%$ ) were retained within the fiber after prolonged exposure to water (curves leveled off). Here, we speculated that at elevated $\mathrm{RH}$, the hydrophilic EGCG might have preferentially interacted with water present in the free volume of the fiber, thereby causing a greater tendency for EGCG to elute during the water extraction step.

\subsubsection{FTIR analysis}

According to the references, the amide $\mathrm{I}$ and II bands are the two most prominent vibrational bands of the protein backbone. The most sensitive spectral region to the protein secondary structural components is the amide I band (1700 - $1600 \mathrm{~cm}^{-1}$ ), which is due almost entirely to the $C=0$ stretch vibrations. The amide $/$ band $\left(1600-1500 \mathrm{~cm}^{-1}\right)$, in contrast, is derived mainly from $\mathrm{N}-\mathrm{H}$ bending and from the $\mathrm{C}-\mathrm{N}$ stretching vibrations. Amide $\mathrm{I}$ is generally believed to consist of a number of underlying bands related to the secondary structure of protein: intermolecular $\beta$-sheets at $1695-1685 \mathrm{~cm}^{-1}$, intramolecular $\beta$-sheets at $1684-1670 \mathrm{~cm}^{-1}, \beta$-turns at $1668-1660 \mathrm{~cm}^{-1}, \alpha$-helices at $1659-1648 \mathrm{~cm}^{-1}$, random coil at $1645-1640 \mathrm{~cm}^{-1}$, intramolecular $\beta$-sheets at $1640-1630 \mathrm{~cm}^{-1}$, 
and intermolecular $\beta$-sheets at $1625-1610 \mathrm{~cm}^{-1}$ (Krimm and Bandekar 1986; Ismail and others 1992; Jackson and Mantsch 1995; Panick and others 1999).

FTIR spectra for freshly spun zein fibers, spun EGCG-containing fibers, and EGCG powder are shown in Figure 14. The position of the amide I band for zein shifted from 1651 to $1649 \mathrm{~cm}^{-1}$ when EGCG was added. Similarly, the amide II band also shifted from 1537 to $1535 \mathrm{~cm}^{-1}$. These shifts implied that the vibration for $\mathrm{C}=\mathrm{O}$ and $\mathrm{N}-\mathrm{H}$ groups of the polyamide backbone were weakened due to the presence of EGCG. An increased intensity was observed on the shoulder of amide I around $1640-1620 \mathrm{~cm}^{-1}$, which is related to $\beta$-sheet structures. Unfortunately, the interpretation of this region is confounded by the presence of EGCG (Figure 14B) and the increased intensity cannot be related to $\beta$-sheet structures. Nevertheless, the band located around $1650 \mathrm{~cm}^{-1}$, which is relatively free from EGCG interference, increased in intensity when EGCG was added, indicating that $a$-helix and random coil fractions increased, perhaps due to the disruption of EGCG for $\beta$-sheet formation during drying. An increase in intermolecular $\beta$-sheet has been reported when zein is dried from aqueous alcohol solution (Mizutani and others 2003; Mejia and others 2007). 


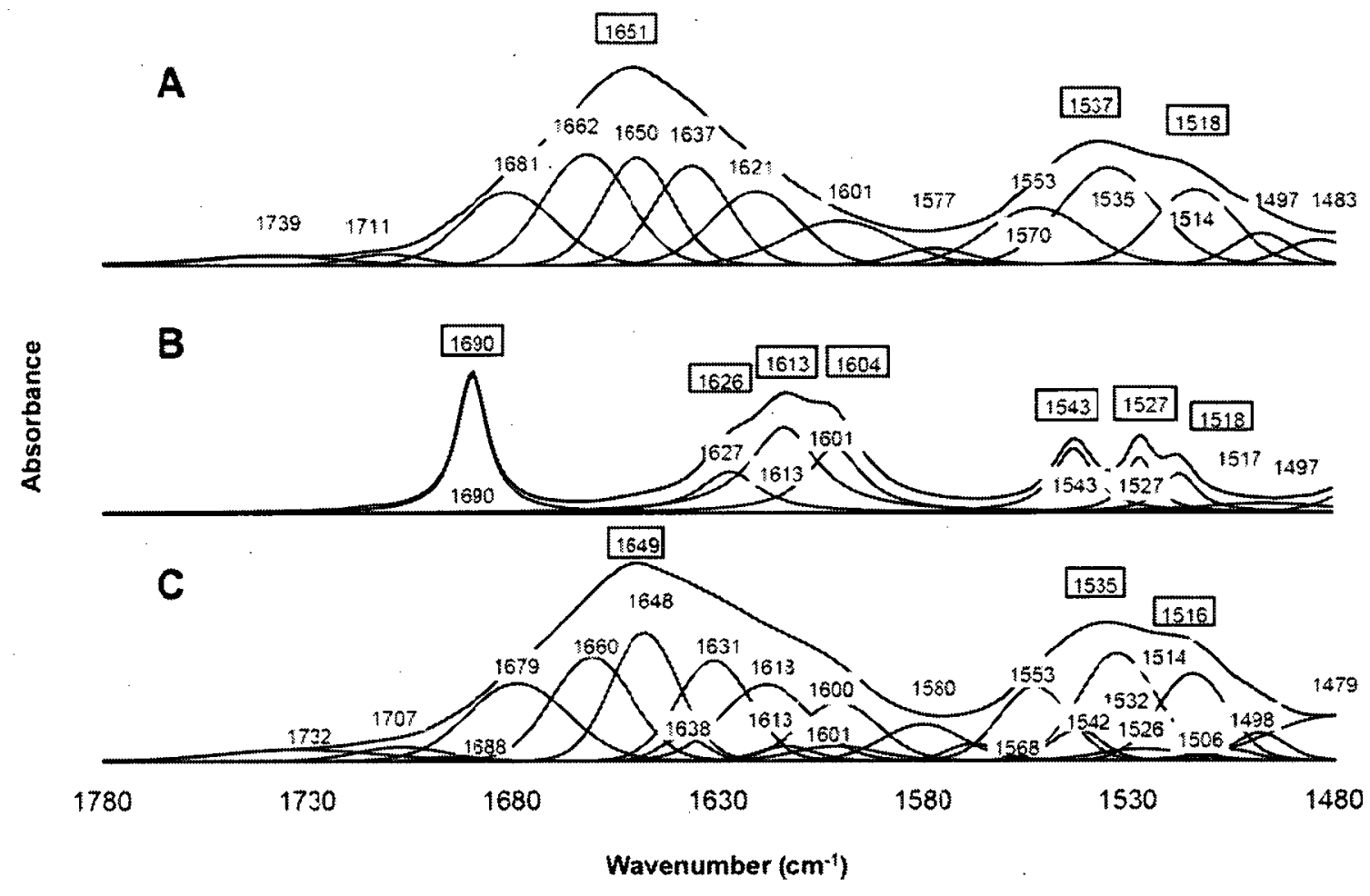

Figure 14 - Representative FTIR spectra for zein fibers alone (A), EGCG powder (B), and zein fibers with 20\% EGCG (C). The spectra for fiber samples were taken immediately after electrospinning before aging (at day 0 ). The fitted curves were based on the peak positions determined from second-derivative of the spectrum followed by Gaussian curve fitting for protein and Lorentzian fitting for $E G C G$.

The IR spectra for zein and EGCG-containing zein fibers conditioned at different $\mathrm{RH}$ are summarized in Figure 15. In the absence of EGCG, aging of fibers at $0 \% \mathrm{RH}$ caused the amide I and II bands to shift to higher wavenumbers (Figure 15A), which may be due to the loss of residual moisture during the 3-day period. At $75 \% \mathrm{RH}$, no detectable shift was observed when the same samples were aged (Figure 15B). In the presence of EGCG, aging at $0 \% \mathrm{RH}$ resulted in many spectral changes (Figure 15C). The presence of EGCG resulted in 
considerable amide I and amide II peak shifts to lower frequencies, indicating that $\mathrm{C}=\mathrm{O}$ and $\mathrm{N}-\mathrm{H}$ groups may have been involved in hydrogen bonding with EGCG. The deconvoluted spectral data for Figure 15C are presented in Figure 16 , showing that intensities of shoulders around $1610 \mathrm{~cm}^{-1}$ increased with time which can be attributed to the increased intermolecular and intramolecular $\beta$ sheet structures (peak area increased from 31 to $36 \%$ of the secondary structures for Amide I). The band located at the shoulder of amide II around 1518 $\mathrm{cm}^{-1}$ band also increased with a concurrent shift to lower wavenumbers as aging time increased. This band has been assigned to the tyrosine $\mathrm{C}-\mathrm{C}$ rings vibration (Arrondo and Goni 1999; Jung 2000; Meersman and others 2002). The shift of this band to lower wavenumber implied that the tyrosine side group $(-\mathrm{OH})$ may have been involved in hydrogen bonding interaction with EGCG or neighboring side groups due to the increased $\beta$-sheet structures. These observations suggested that the secondary structure of protein were altered due to the interaction with EGCG, further supporting the hypothesis that the enhanced EGCG retention observed under dry condition (Figure 13) was not due to the conformational change of zein alone, but was caused by the cooperative interaction of zein with EGCG. 

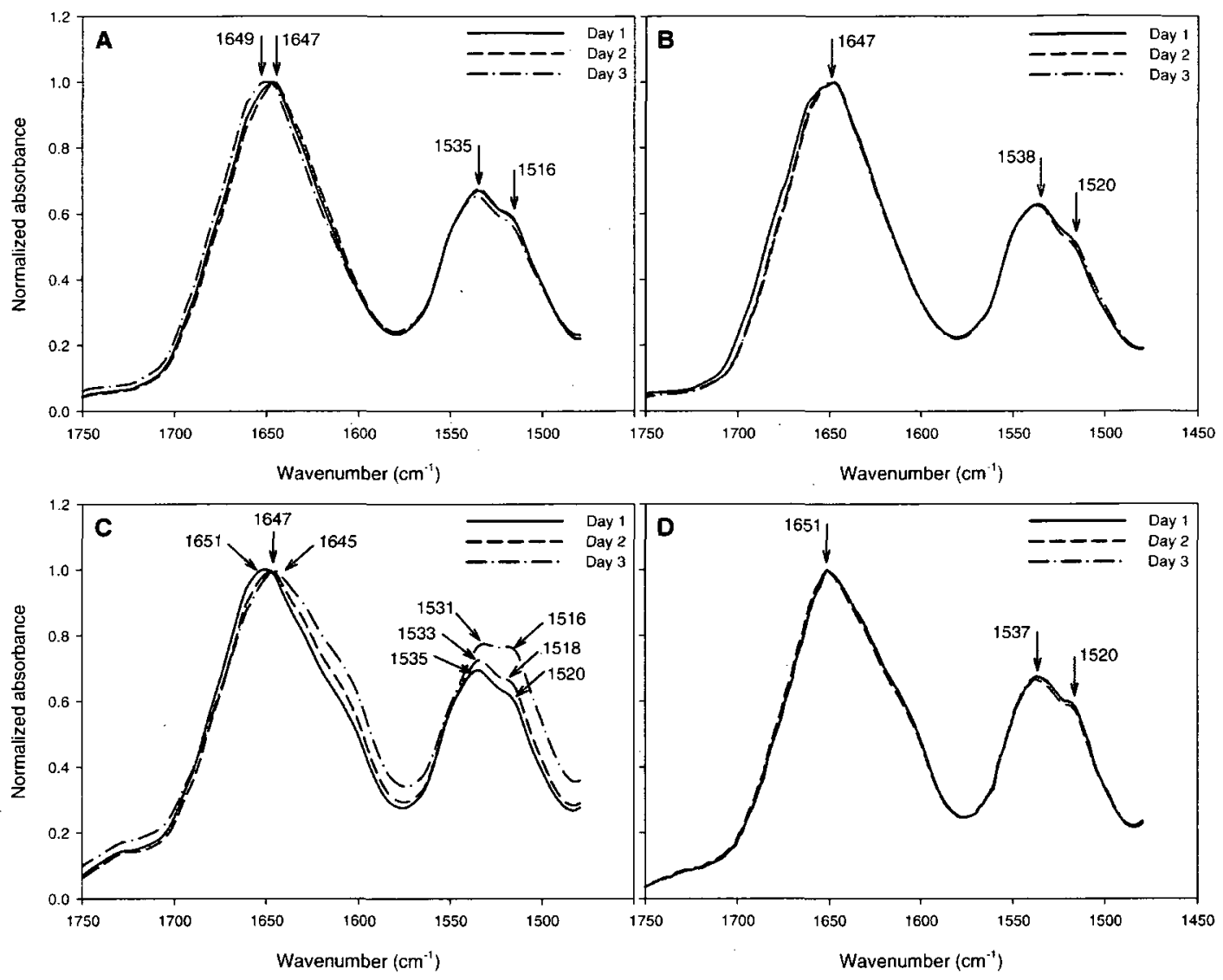

Figure 15 - FTIR spectra for zein and EGCG-containing zein fiber aged in 0\% or $75 \% R H$ conditions at $21 \pm 2{ }^{\circ} \mathrm{C}$. A: zein fibers at $0 \% \mathrm{RH}$; B: zein fibers at $75 \%$ $R H$; C: zein + EGCG fibers at O\% RH; D: zein + EGCG fibers at $75 \% R H$.

Interestingly, at $75 \% \mathrm{RH}$ (Figure 15D), the presence of EGCG did not induce observable changes on both amide I and II bands, implying that aging under elevated humidity did not induce substantial changes in protein conformation. Under this condition, the preferential interaction of $\mathrm{C}=\mathrm{O}$ and $\mathrm{N}-\mathrm{H}$ with the absorbed water might have precluded the EGCG from interacting with these groups. This observation also suggested that hydrogen bonding between EGCG and zein was important, at least initially during dry treatment, for facilitating the subsequent changes in zein secondary structures which enhanced 
the EGCG retention within the zein fiber when immersed in water. Such changes may result in the development of hydrophobic interactions between benzene rings of EGCG and hydrophobic amino acid residues of zein and/or physical encapsulation of EGCG within the zein fiber. The latter hypothesis is based on the observations that $75 \% \mathrm{RH}$ condition did not induce protein conformational changes (Figure 15D), but the fibers still retained about $67 \%$ of EGCG after 60 min immersion in water (Figure $13 ; 75 \% \mathrm{RH}$, day 3 ).

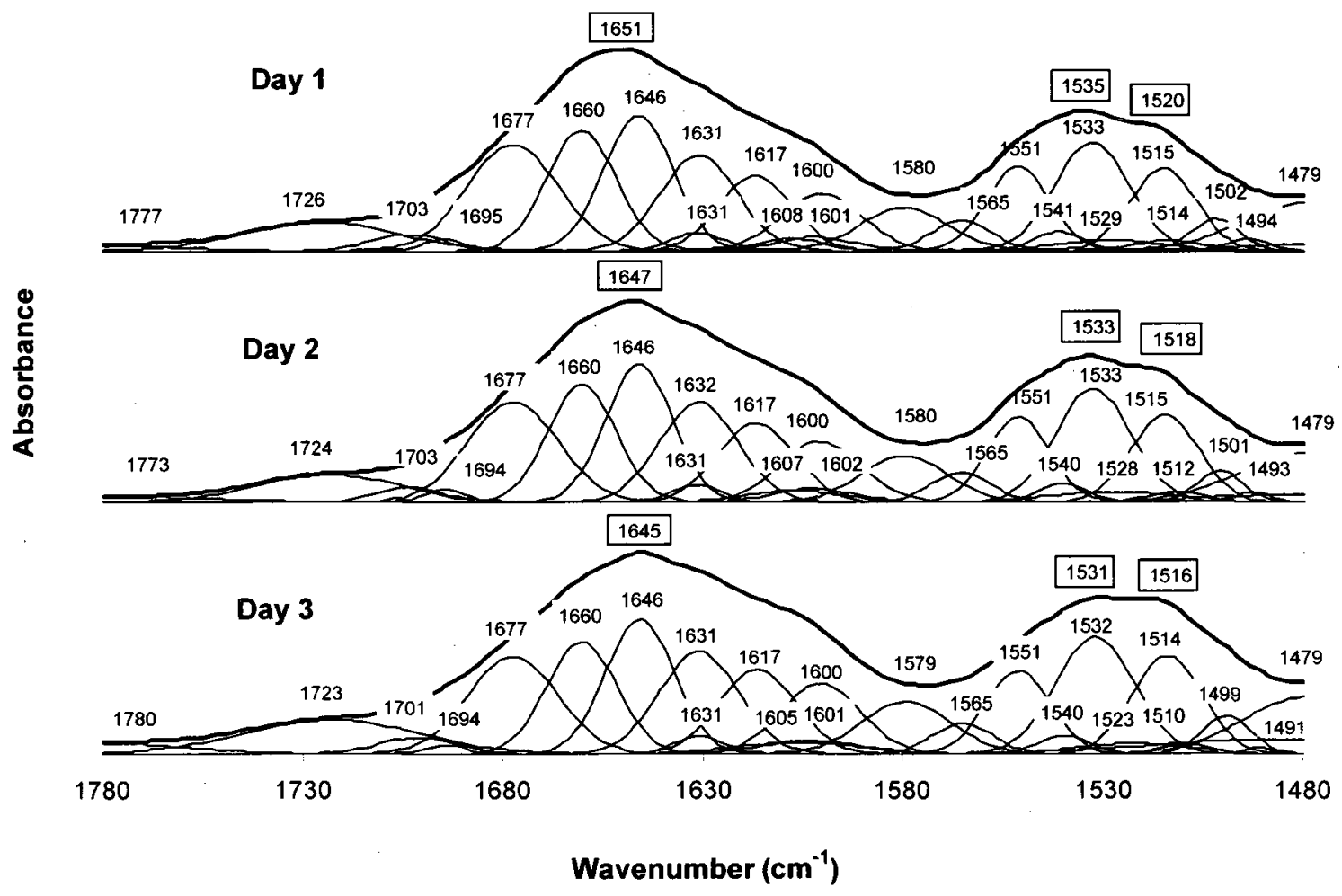

Figure 16 - FTIR spectra and deconvoluted bands for EGCG-containing zein fibers aged in $0 \% R H$ conditions for 3 days at $21 \pm 2{ }^{\circ} \mathrm{C}$. 


\subsection{Conclusions}

In this study, the zein fibers resisted solubilization in water, although swelling and plasticization were apparent after the water treatment. The efficacy of zein fibers was tested for stabilization of a green tea polyphenol, (-)epigallocatechin gallate (EGCG), by incorporating the EGCG in zein fiber-forming solutions. Both relative humidity and aging time after spinning were important in determining the stability of EGCG upon subsequent contact with water. Freshly spun fibers were less effective at immobilizing the EGCG upon immersion in water ( $82 \%$ remaining percentage) as compared to fibers that were aged at $0 \%$ relative humidity for at least $1 \mathrm{~d}$ (>98\% remaining percentage) before water immersion. FTIR spectra and curve fitting suggested that hydrogen bonding, hydrophobic interactions, and possibly physical encapsulation are involved in enhancing the retention of EGCG in zein fibers that are immersed in water. More studies are currently underway to characterize the stability of EGCG immobilized in zein fibers under various conditions typically encountered in food products. 


\section{THE STABILITY OF EGCG ENCAPSULATED IN ELECTROSPUN ZEIN FIBERS}

\subsection{Introduction}

Tea catechins, polyphenol components extracted from green tea, have received increased attentions over the past few years, due to their strong antioxidative activity which has been associated with the prevention of diseases such as heart disease and cancers (Higdon and Frei 2003; Zaveri 2006; Wang and others 2008). The main catechins identified in green tea are (-)epigallocatechin (EGC), (-)-epigallocatechin gallate (EGCG), which can be converted to their (-)-gallocatechin gallate (GCG) and (-)-gallocatechin (GC) epimers, respectively (Wang and others 2008). The chemical structures of epicatechins and non-epicatechins differ at the 2 and 3 positions. The epimer adopts $2 R, 3 R(2,3-c i s$,$) , while the nonepimer 2 S, 3 R(2,3$-trans) configurations (Figure 17). The three adjacent hydroxyl (Miyoshi and others) groups at positions C-3', $-4^{\prime}$ and $-5^{\prime}$ on the $B$ ring of (-)-EGCG, (-)-EGC, (-)-GCG and (-)-GC are effective in scavenging free radicals. Moreover, catechins with gallate group at C3 have stronger scavenging effects than non-gallate catechins (Salah and others 1995; Chen and Chan 1996; Basu and Donaldson 2003; Wang and others 2008).

The stability of catechin is $\mathrm{pH}$ and temperature dependent. In alkaline solutions $(\mathrm{pH}>7)$, tea catechins are very unstable and degraded almost completely within a few minutes. Whereas in acidic solutions $(\mathrm{pH}<4)$, catechins are relatively more stable (Zhu and others 1997; Chen and others 1998; Wang and others 2008). The degradation of catechins is mainly affected by 
temperature, media in which it is exposed to and oxygen (Wang and others 2008). Wang and others summarized that the degradation and epimerization of tea catechin could occur simultaneously during thermal processing. Besides degradation and epimerization commonly observed at high temperature, catechins have also been found to degrade slightly at ambient temperature (Wang and others 2008). Also, Zhu and others have elucidated the absorption mechanism of dietary green tea catechins (GTCs) in the intestine of rats and their free-radical scavenging capacity at various $\mathrm{pH}$ conditions. They further studied the stability of these GTC isomers in acidic $\mathrm{pH}$ of stomach and slightly alkaline intestinal conditions. For the conditions between $\mathrm{pH} 4$ to 8 , they concluded that the lower pH had greater stability (Zhu and others 1997).

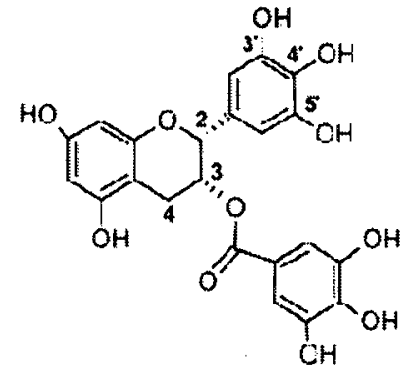

(-)GCG

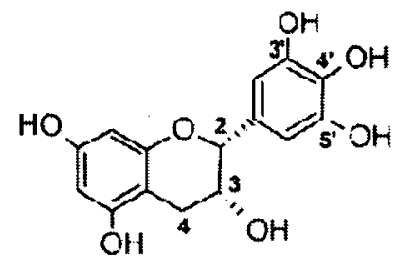

(-)ECC

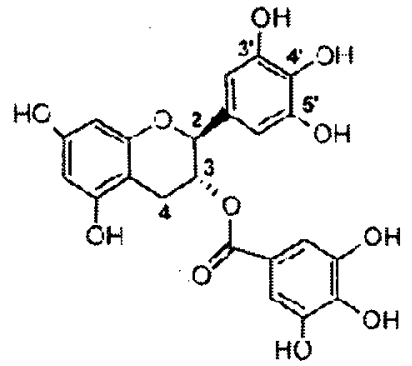

(-)-EGCG<smiles>C[14C@@H]1Oc2cc(O)cc(O)c2O[C@@H]1c1cc(O)c(O)c(O)c1</smiles>

(-) -GC

Figure 17 - Chemical structures of green tea catechins: (-)-epigallocatechin gallate (EGCG), (-)-gallocatechin gallate (GCG), (-)-epigallocatechin (EGC) and (-)-gallocatechin (GC) (Wang and others 2008). 
EGCG, the most abundant and biologically active compound in tea catechins, has been extensively studied for its antioxidant activities related to disease prevention from carcinogen-induced skin, lung, forestomach, esophagus, duodenum, liver and colon tumors in rodents (Zimeri and Tong 1999). However, its stability can be affected by a number of deleterious factors, such as $\mathrm{pH}$, temperature, oxygen and UV light (Chen and others 2001; Sang and others 2005). Some researchers have suggested that EGCG solutions can be stabilized by using metal scavengers (e.g., ethylenediaminetetraacetic acid, EDTA), antioxidants (e.g. ascorbic acid), maintaining a pH below or close to neutral, and by keeping the temperature cold (below $4{ }^{\circ} \mathrm{C}$ ) during storage (Wang and others 2006). Chen and others demonstrated that the presence of ascorbic acid may prevent the degradation and epimerization of EGCG in the intestine where the $\mathrm{pH}$ is neutral or slightly alkaline (Kiatgrajai and others 1982). They also concluded that ascorbic acid did not increase the total GTCs, but it reduced the rate of EGCG degradation (Chen and others 1998).

Our previous results showed that EGCG-containing electrospun fibers resisted solubilization in water, and that the maximal EGCG retention in water was achieved when the zein fiber was aged for at least 1 day at $0 \% \mathrm{RH}$. This section focuses on determining if the electrospun fibers are effective in stabilizing EGCG in model solutions with acidic, neutral and alkaline conditions at refrigerated and thermal processing conditions. The EGCG-containing zein fibers were tested in models solutions at $\mathrm{pH} 3,5,7$ and 9 to simulate different $\mathrm{pH}$ conditions found in food products. The thermal stability of encapsulated EGCG in 
zein fibers was also evaluated at $4,25,60$, and $100{ }^{\circ} \mathrm{C}$ to replicate various thermal processing and storage conditions. Overall, the specific objectives were to determine the stability of EGCG in complex aqueous model system, to conduct the degradation reaction of EGCG encapsulated in electrospun zein fibers and to investigate the rates for formation of the degraded compounds, as a function of $\mathrm{pH}$ and temperature.

\subsection{Materials}

Zein was purchased from Sigma-Aldrich (St. Louis, Mo., U.S.A.) and was used as received, without further purification. Ethanol of $100 \%$ purity was supplied by Commercial Alcohols (Brampton, Ont., Canada). GCG and EGC standards were purchased from Sigma-Aldrich (St. Louis, Mo., U.S.A.). Purified EGCG powder, extracted from green tea, was donated by Mitsui Norin Co., Ltd. (Fujieda, Japan). Ascorbic acid, formic acid, acetonitrile, disodium hydrogen phosphate $\left(\mathrm{Na}_{2} \mathrm{HPO}_{4}\right)$ and hydrochloric acid $(\mathrm{HCl})$ were purchased from Fisher Scientific (Ottawa, Ont., Canada). Potassium hydrogen phthalate (KHP) and sodium hydroxide $(\mathrm{NaOH})$ were purchased from Sigma-Aldrich (St. Louis, Mo., U.S.A.). 


\subsection{Methods}

\subsubsection{Electrospinning}

EGCG-containing zein solution, containing $20 \%(\mathrm{w} / \mathrm{w})$ zein with $20 \%(\mathrm{w} / \mathrm{w}$, in zein) EGCG dissolved in $70 \%(w / w)$ aqueous ethanol, was electrospun as described in section 4.3.1.

\subsubsection{Morphology of electrospun fibers}

Freshly electrospun fibers were examined using a scanning electron microscope (SEM) as described in section 4.3.2.

\subsubsection{Preparation of the aqueous model systems}

Four different buffer solutions were prepared: $\mathrm{pH} 3$, potassium hydrogen phthalate (KHP, 0.1 M) and hydrochloric acid $(\mathrm{HCl}, 0.1 \mathrm{M})$; $\mathrm{pH} 5, \mathrm{KHP}(0.1 \mathrm{M})$ and sodium hydroxide $(\mathrm{NaOH}, 0.1 \mathrm{M}) ; \mathrm{pH} 7$, disodium hydrogen phosphate $\left(\mathrm{Na}_{2} \mathrm{HPO}_{4}, 0.1 \mathrm{M}\right)$ and $\mathrm{HCl}(0.1 \mathrm{M}) ; \mathrm{pH} 9, \mathrm{Na}_{2} \mathrm{HPO}_{4}(0.1 \mathrm{M})$ and $\mathrm{HCl}(0.1 \mathrm{M})$.

\subsubsection{Experimental design for stability studies}

A $4 \times 4$ full factorial experiment was conducted to study the effect of $\mathrm{pH}$ and temperature on the stability of EGCG. The EGCG containing fibers were placed in a vial and submerged in the model aqueous solutions $(\mathrm{pH} 3,5,7$ or 9$)$ as outlines in section 6.3 .3 and exposed to constant temperature $(4,25,60$ or 100 ${ }^{\circ} \mathrm{C}$ ). For the heat treatment at 60 and $100{ }^{\circ} \mathrm{C}$, the sealed vials containing the fibers were heated in constant temperature water baths (Haake W26 Thermo 
Fisher Scientific, Waltham, Mass., U.S.A.) without mechanical shaking at 60 and $100 \pm 0.5{ }^{\circ} \mathrm{C}$. After the thermal treatments, the vials were immediately removed and placed in an ice bath for 90 seconds to cool to $4{ }^{\circ} \mathrm{C}$. In order to prevent further degradation of EGCG, ascorbic acid as an optimal agent was added to reduce $\mathrm{pH}$ of the solution to 3 before dissolving zein fibers by adding ethanol. In

order to replicate the stability of EGCG at 4 and $25{ }^{\circ} \mathrm{C}$, the solutions containing fibers were stored at 4 and $25{ }^{\circ} \mathrm{C}$ without heat treatment. The extraction and analysis of EGCG encapsulated in the fibers were performed under ambient conditions.

\subsubsection{HPLC analysis}

Analysis of (-)-EGCG and its degraded compounds was performed using a HPLC equipped with a photodiode array UV detector (2695/2696 Waters, Milford, Mass.; U.S.A.) and a C18 reversed phase column $(150 \mathrm{~mm} \times 3.9 \mathrm{~m} / 4 \mu \mathrm{m}$, Waters, state, country). The HLPC was equipped with an auto-injector and controlled at $20 \pm 1{ }^{\circ} \mathrm{C}$. The mobile phases were $0.1 \%(\mathrm{v} / \mathrm{v})$ formic acid in water (eluent A) and $100 \%$ acetonitrile (eluent B) used at a constant flow rate of 0.8 $\mathrm{mL} / \mathrm{min}$. The elution gradient applied was: 0 - $10 \mathrm{~min}, 10 \% \mathrm{~B} ; 10$ - $30 \mathrm{~min}$, linear gradient from 10 to $30 \% B ; 30-40 \min , 30-60 \% B ; 40-45 \min 60-10 \% B$; $45-55 \min 10 \%$ B. The elution time for a single run took 55 min and the postrun time was $10 \mathrm{~min}$. The sample injection volume was $20 \mu \mathrm{L}$. Detection was carried out at $275 \mathrm{~nm}$ wavelength. Retention times for (-)-EGC, (-)-EGCG and (-)-GCG were $6.6 \mathrm{~min}, 17.1 \mathrm{~min}$ and $19.2 \mathrm{~min}$, respectively (Figure 18). 


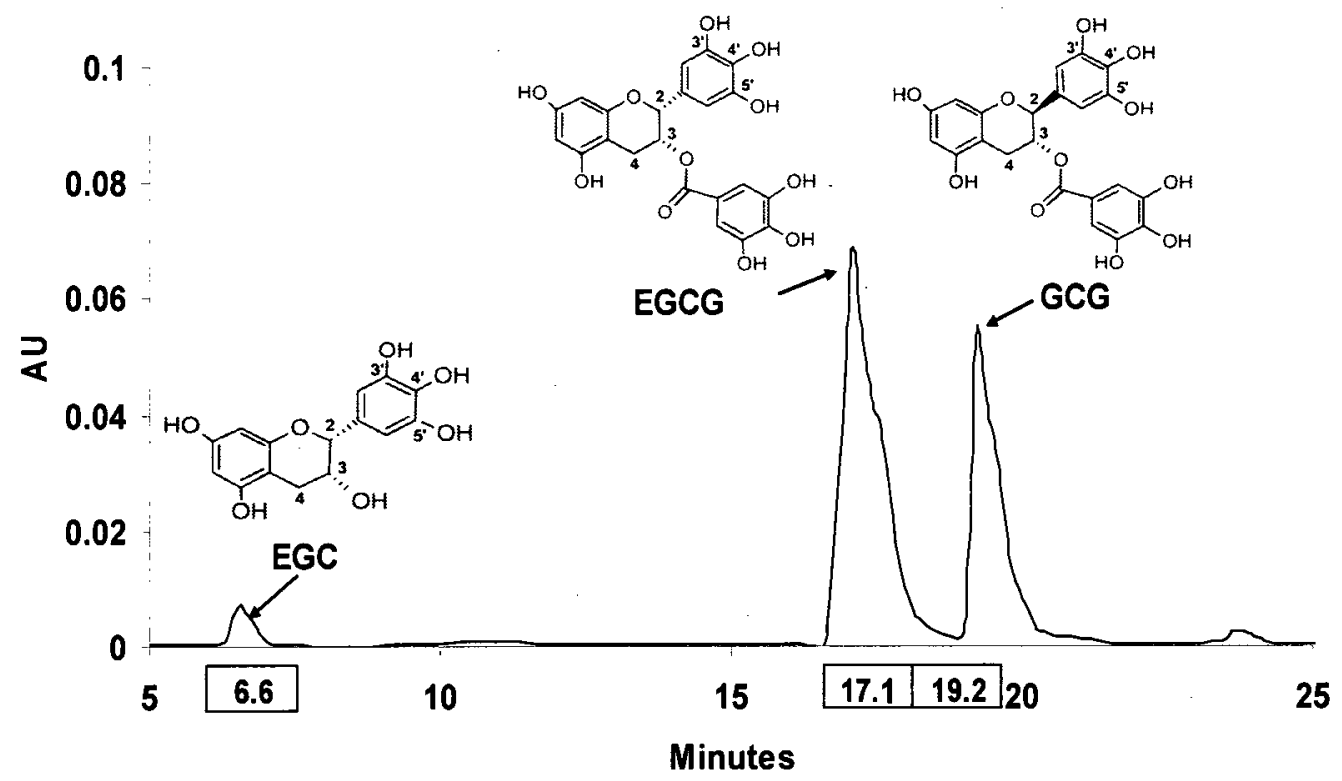

Figure 18 - HPLC profile of EGC, EGCG and GCG standards was detected at $274.5 \mathrm{~nm}$ respectively.

HLPC grade pure standards of (-)-EGC, (-)-EGCG and (-)-GCG were freshly prepared in aqueous solution. Five concentrations of each standard solution were made at $0.02,0.04,0.06,0.08$, and $0.10 \mathrm{mg} / \mathrm{mL}$. Calibration curves for the three standards were linear with the coefficients of determination $\left(R^{2}\right)$ close to unity (see Appendix in Section 10).

The amount of EGCG encapsulated within the fibers $\left(W_{E G C G_{t}}\right)$ are calculated by subtracting exactable EGCG $\left(W_{E G C G e}\right)$ from the total (residual and extractable) EGCG $\left(W_{E G C G r}\right)$ determined as the following extractable process. The equations are described as followed:

$$
W_{E G C G_{i}}=W_{E G C G_{r}}-W_{E G C G_{e}}
$$




$$
\begin{gathered}
W_{E G C G_{e}}=c \times L_{3} \\
W_{E G C G_{r}}=c \times L_{6} \\
L_{6}=\left(L_{1}+L_{2}+L_{4}+L_{5}\right)-L_{3}
\end{gathered}
$$

unlike preliminary stability study, where $L_{3}$ is the determined volume for extractable EGCG, $L_{6}$ is the volume of remaining extractable and residual EGCG, which is subtracted the determined volume of extractable EGCG $\left(L_{3}\right)$ from the total volume of aqueous solutions (buffer solution with the range of pH4 - $9\left(L_{l}\right)$, ascorbic acid for preventing further degradation after treatments $\left(L_{2}\right)$, aqueous ethanol for dissolving zein fibers $\left(L_{4}\right)$ and water for precipitating zein protein $\left(L_{5}\right)$. The procedure is shown in Figure 19. $W_{E G C G o}$ is the initial amount of EGCG added in the polymer solution, calculated by multiplying the weight of electrospun fibers with percentage of EGCG containing in the fibers, as shown:

$$
W_{E G C G_{o}}=W_{f} \times\left(\frac{W_{E G C G_{o}}{ }^{\prime}}{W_{Z e i n}+W_{E G C G_{o}}{ }^{\prime}} \times 100 \%\right)
$$

therefore, the percentage of residual EGCG $(p)$ is calculated as the ratio of the determined weight of EGCG $\left(W_{E G C G i}\right)$ divided by the calculated weight of initial EGCG $\left(W_{E G C G_{0}}\right)$ based on the amount added in the formulation,

$$
p \%=\frac{W_{E G C G_{i}}}{W_{E G C G_{o}}} \times 100 \%
$$


The calculation procedures for determinations of EGCG degraded products including GCG, EGC and etc., are the same as the calculation methodology for encapsulated EGCG in fibers and exactable EGCG from fibers. Also, EGCG powders and their degraded compounds as the controls are processed the same procedures of various treatments, including extraction, HPLC determination and calculation as the process of EGCG samples described above. The percentage of EGCG controls and their degraded constitutes are calculated as ratios of the determined weight of EGCG controls divided by the weight of initial EGCG added in the formulation.

\subsubsection{EGCG extraction and determination}

Results from the preliminary stability study presented in Section 5 suggested that maximal EGCG retention in fiber was achieved in water when the zein fibers were aged for at least 1 day at $0 \% \mathrm{RH}$. Thus, the same aging treatment was used before subjecting the fibers to this stability study. To evaluate the stability of the encapsulated EGCG, aged fibers were submerged in solutions of various $\mathrm{pH}$ ranging from $4-9$ and treated at $4-100{ }^{\circ} \mathrm{C}$. After the treatments, $\mathrm{pH}$ values of solutions were decreased to 3 by adding ascorbic acid to prevent further degradation. Then, the resulting supernatant with EGCG was examined by HPLC for "extractable EGCG". To determine the mixture fraction of "residual" and the rest of "extractable" EGCG, the zein fibers were dissolved in aqueous ethanol, followed by adding water gradually to precipitate the zein. The whole process was schematically represented in Figure 17. In order to release all the EGCG, the resulting solution was centrifuged, filtered, and analyzed by HPLC. 
The EGCG remaining was calculated as a ratio of the determined EGCG divided by the amount of EGCG used in the formulation (Equation 6.5). All the experiments were performed in at least triplicate, and data were presented as means \pm standard deviations.

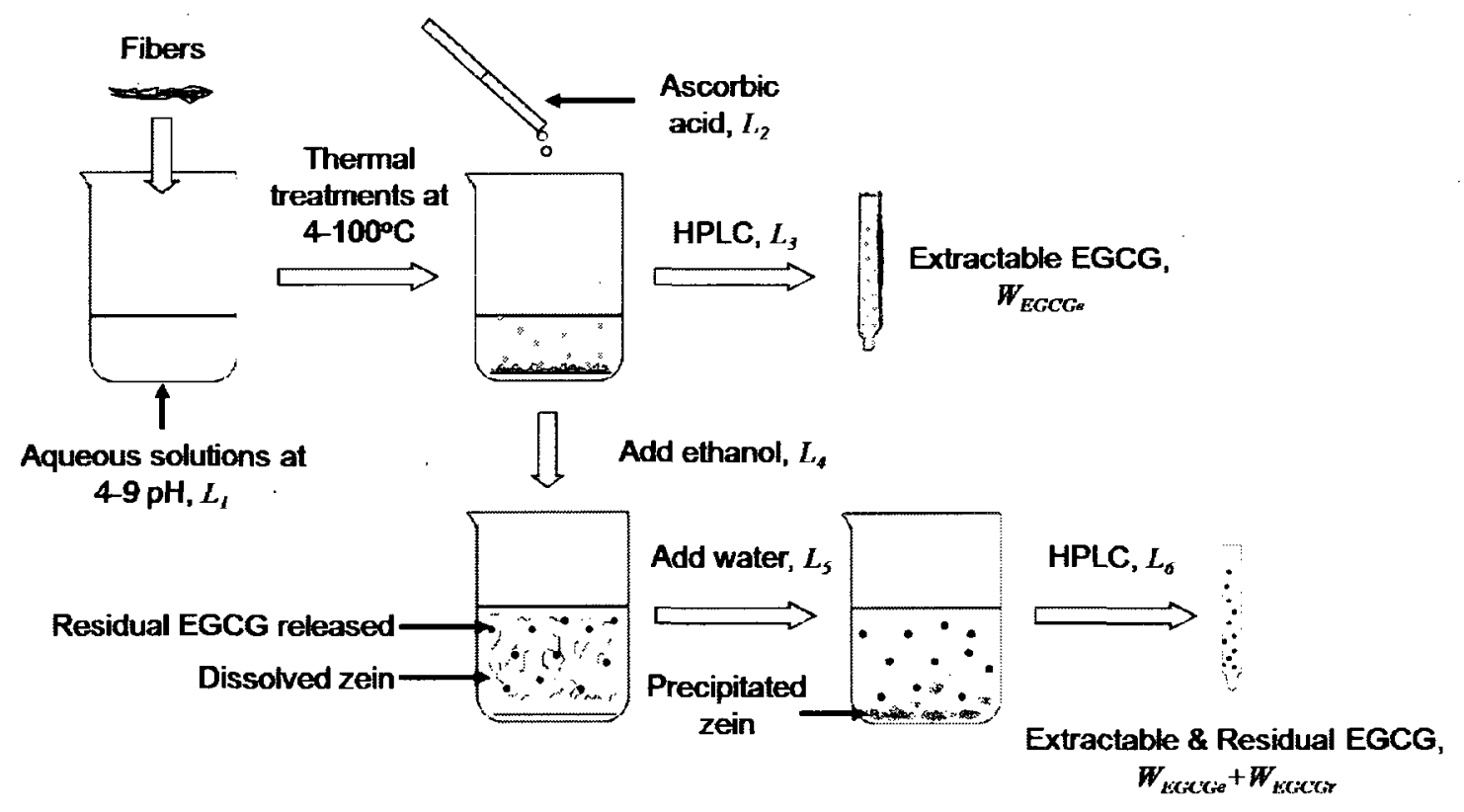

Figure 19 - Schematic representation of EGCG extraction process for the stability study.

\subsection{Results and Discussions}

\subsection{1 pH effects on EGCG stability}

The degradation of fiber-encapsulated EGCG increased with increasing $\mathrm{pH}$. At $\mathrm{pH} 9$, there was more than $80 \%$ EGCG loss after 300 minute treatment when held at under ambient conditions. However, the unencapsulated EGCG control samples dropped close to 0 after 300 minute exposure to $\mathrm{pH} 9$ solution (Figure 20A). By contrast, the residual EGCG in aqueous system at $\mathrm{pH} 7$ was $40 \%$ after 
exposure for 2 day (Figure 20B). The enhanced degradation of EGCG is believed to be caused by the proton-semiquinone free radicals occurring at neutral or alkaline $\mathrm{pH}$, making it more susceptible to degradation and deformation (Wang and others 2006).

In acidic solution ( $\mathrm{pH} 5$ ), the percentage of EGCG remaining in the fibers was $60 \%$, which was considerably higher than in the alkaline solution (Figure 20C). Similar observations were reported by other researchers in other nonencapsulated systems (Yoshioka and others 1991; Komatsu and others 1992; Zhu and others 1997; Zimeri and Tong 1999). At pH 3, the stability of the encapsulated-EGCG was further enhanced, resulting in greater than $90 \%$ retention after exposure for up to 2 days. However, about $40 \%$ of the unencapsulated EGCG control was degraded under the same condition. The stability observations show that EGCG was vulnerable to degradation at ambient condition in aqueous solution of $\mathrm{pH}$ ranging from 3 to 9 . However, higher amounts of EGCG were recovered than controls in solutions with various $\mathrm{pH}$, respectively, when the polyphenol was encapsulated in zein fiber, indicating that electrospun zein fiber could improve the stability of EGCG. The degradation rate of EGCG was increased with an increase of $\mathrm{pH}$. The higher the $\mathrm{pH}$ values of the medium, the greater the amount of EGCG that degraded.

The stability data show that EGCG was vulnerable to degradation at ambient conditions in aqueous solution of $\mathrm{pH}$ ranging from 3 to 9 . However, high amounts of EGCG were recovered when the polyphenol was encapsulated in zein fiber and tested under the same condition, indicating that electrospun zein 
fiber could improve the stability of EGCG. The degradation rate of EGCG was increased with an increase of $\mathrm{pH}$ (Figure 20).
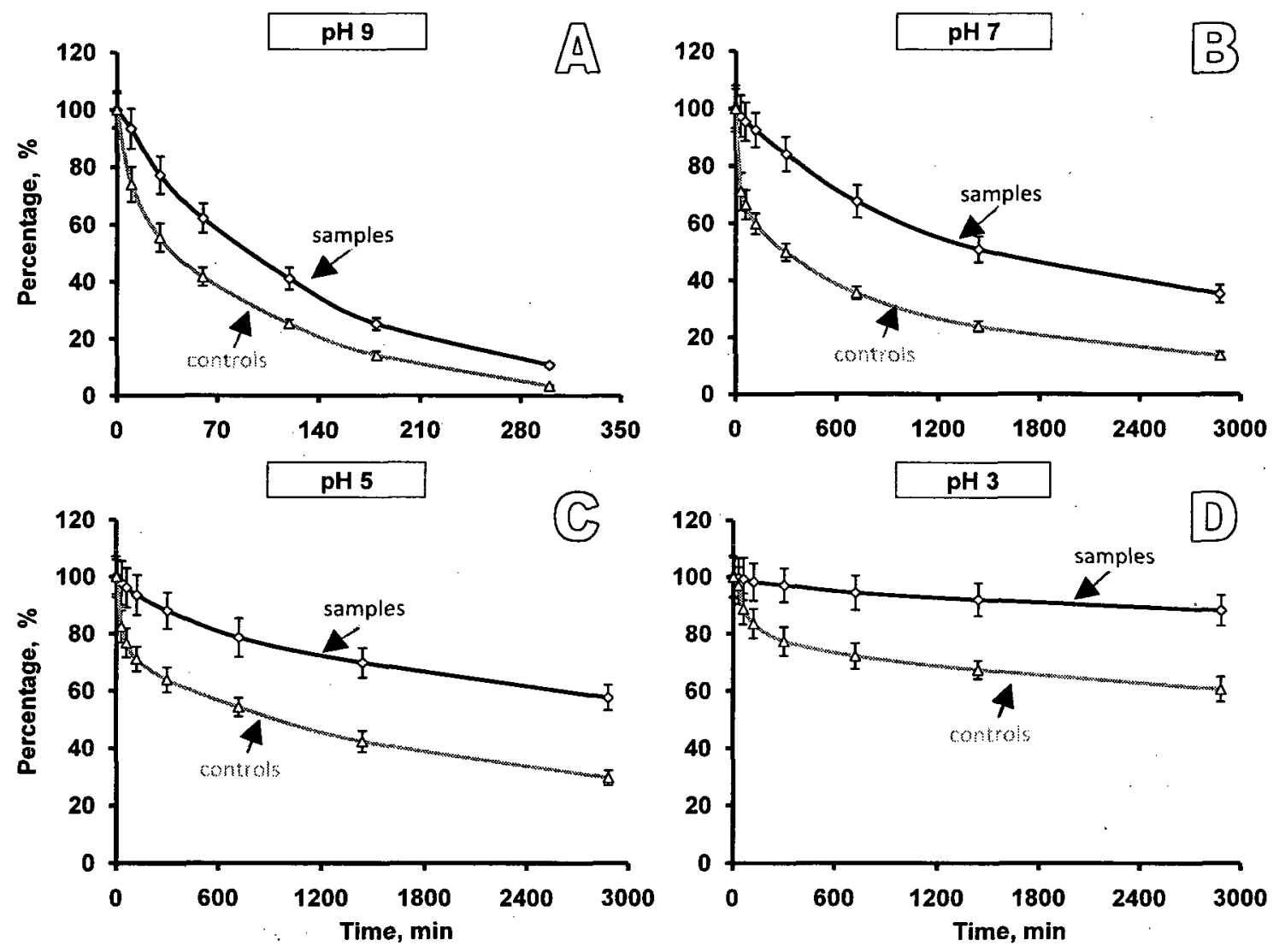

Figure 20 - Remaining percentage of residual EGCG encapsulated by electrospun zein fibers at $25{ }^{\circ} \mathrm{C}$ in various $\mathrm{pH}$, compared to percentage of controls. A: $p H$ 9; $\mathbf{B}: \mathrm{pH}$ 7; C: $\mathrm{pH} 5$ and $\mathbf{D}: \mathrm{pH} 3$.

\subsubsection{Temperature effects on EGCG stability}

Temperature is one of the factors that affect the stability of EGCG. Figure 21 compared the stability of EGCG encapsulated by electrospun zein fibers with unencapsulated control samples in $\mathrm{pH} 7$ aqueous solution at different temperatures. As shown, the degradation of EGCG increased with increasing 
temperature. At $4{ }^{\circ} \mathrm{C}, 75 \%$ EGCG was retained when the polyphenol was encapsulated in electrospun zein fibers for up to 2 days, whereas all EGCG was degraded at $100{ }^{\circ} \mathrm{C}$ within 1 hour.

In Figure 21D, about 60\% of EGCG (control) was lost when immersed in solution with $\mathrm{pH} 7$ for 4 days at $4{ }^{\circ} \mathrm{C}$. In contrast, EGCG encapsulated in zein fibers remained $75 \%$ for the same period at $4{ }^{\circ} \mathrm{C}$. At ambient temperature (25 ${ }^{\circ} \mathrm{C}$ ), almost half of the encapsulated EGCG in zein fibers was retained. On the other hand, only about $15 \%$ of the unencapsulated EGCG was recovered under the same condition (Figure $21 \mathrm{C}$ ). At $70^{\circ} \mathrm{C}$ and after 1.5 hours of exposure in $\mathrm{pH}$ 7 buffer solution (Figure 21B), although EGCG in both the encapsulated and control samples were completed lost, the amount of residual EGCG in fibers was about twice as compared to the controls, indicating the EGCG stabilizing effect of the electrospun zein fibers. In Figure 21A, EGCG was completely degraded at $100{ }^{\circ} \mathrm{C}$ in the solution with $\mathrm{pH} 7$, suggesting that the higher temperature adopted, the greater the degradation reaction. This led us to speculate that the thermal treatment would mainly cause the epimerization. The observation was in the agreement with Chen (Chen and others 2001). And also, no significant differences between the percentages of EGCG encapsulated in fibers and EGCG controls, both treated in aqueous system with $\mathrm{pH} 7$ at $100{ }^{\circ} \mathrm{C}$. After two hours treatments, there were no or little amounts of EGCG observed and all the EGCG was totally degraded in solution with $\mathrm{pH} 7$, this observation was possibility caused by the fact that EGCG corresponding epimers, degraded compounds and other diffused substitutes, were simultaneously produced during processing. The 
degradation of EGCG encapsulated in fibers might be related to epimerization of EGCG to GCG, degradation to other compounds, and diffusion from the fibers into the surrounding solution.
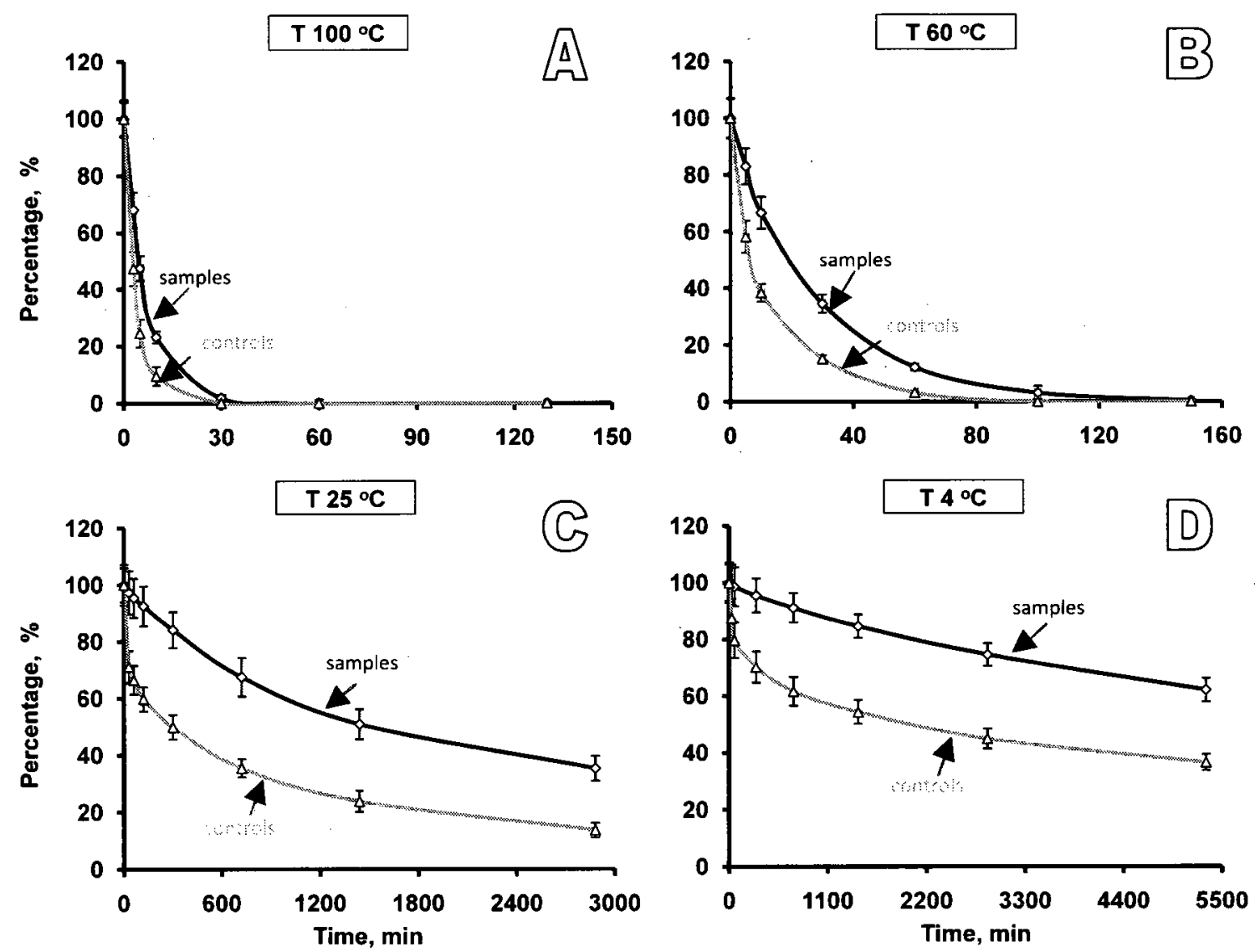

Figure 21 - Remaining percentage of residual EGCG encapsulated by electrospun zein fibers in aqueous system with $\mathrm{pH} 7$ at different temperature, compared to the percentage of controls. A: $100^{\circ} \mathrm{C}$; B: $60^{\circ} \mathrm{C}$; C: $25^{\circ} \mathrm{C}$ and D: 4 ${ }^{\circ} \mathrm{C}$.

\subsubsection{EGCG epimerization}

The percentage of EGCG remaining and the percentage of GCG formed for the encapsulated and control samples under different $\mathrm{RH}$ and temperature 
conditions are summarized in Figure 22. As shown, the rates of degradation for EGCG encapsulated in zein were slower as compared to the control samples, indicating that the electrospun zein fibers did improve the stability of EGCG. While the encapsulated EGCG decrease with time, an increase of GCG was detected. For the unencapsulated EGCG control samples, its epimerization resulted in the formation of GCG which first increased to a maximum concentration and then declined (Figure 22B, D and F). The presence of maxima in GCG profiles indicated that the non-epimer was also susceptible to degradation in the aqueous solution. This epimerization trend was similar to the observation occurred in the solution with $\mathrm{pH} 5$ at $120^{\circ} \mathrm{C}$ in the report by Seto and others (1997). In general, the lower initial rate of GCG formation and the absence of maxima in the GCG profile appeared to suggest that the electrospun zein fibers stabilize not only EGCG but also its GCG epimer GCG under the tested conditions.

The effect of temperature on the epimerization reaction is evident when comparing Figure 22B and Figure 22D. Here, higher concentrations of GCG were detected within the first 90 min of exposure in $\mathrm{pH} 9$ solution at $100^{\circ} \mathrm{C}$ than at 4 ${ }^{\circ} \mathrm{C}$, indicating that the higher the temperature, the faster the epimerization of EGCG to GCG. Moreover, the maximal concentration occurred sooner at $100^{\circ} \mathrm{C}$ ( $25 \mathrm{~min})$ as compared to $4^{\circ} \mathrm{C}(\sim 300 \mathrm{~min})$. This led us to believe that thermal treatment can accelerate both the conversion of EGCG to GCG, as well as the degradation of GCG. This observation is in the agreement with Chen, Komatsu, Xu, Wang and others (Komatsu and others 1992; Chen and others 2001; Xia and 
Xu 2005; Wang and others 2006). Moreover, in Figure 22B, around 3\% GCG of total EGCG applied to fibers was still epimerized from EGCG encapsulated in fibers at $4{ }^{\circ} \mathrm{C}$, suggesting that the epimerization of EGCG could still take place at refrigeration temperature, although at much lower rate.

The effect of $\mathrm{pH}$ can be observed by comparing Figure 22D and $\mathrm{F}$. As shown, at the same temperature $\left(100{ }^{\circ} \mathrm{C}\right)$, higher amounts of $\mathrm{GCG}$ were observed in the solution with $\mathrm{pH} 9$ than $\mathrm{pH}$ 3. Also, the GCG profile for the control sample reached the maximal concentration earlier and degraded faster for $\mathrm{pH} 9$ solution than $\mathrm{pH} 3$ at the same temperature. These results suggested that elevated $\mathrm{pH}$ can accelerate the epimerization, as well as the degradation of the GCG epimer. 

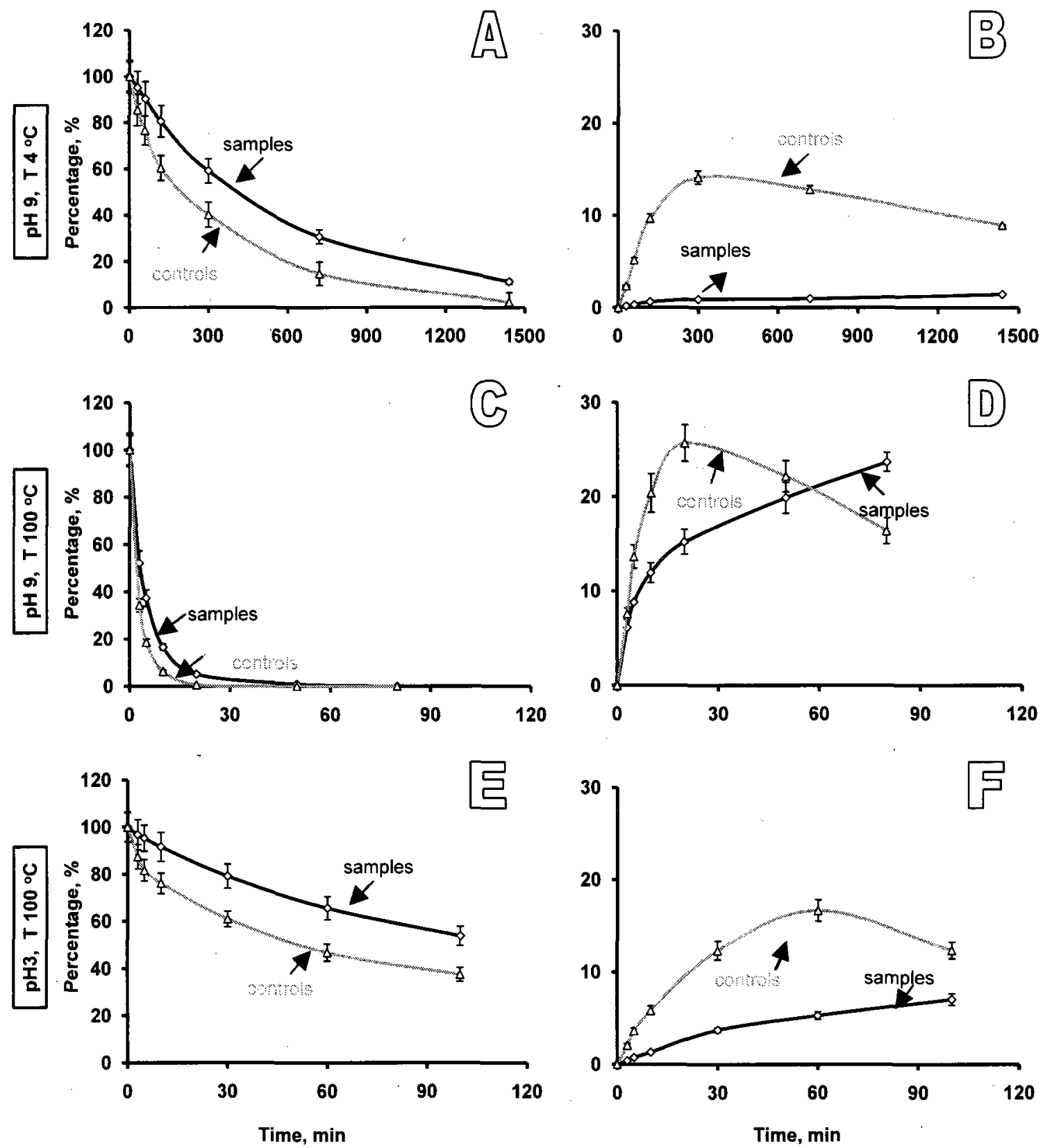

Figure 22 - Percent remaining of residual EGCG and its epimer, GCG, in electrospun zein fibers as compared with the percentage of EGCG control and its epimer under various $\mathrm{pH}$ and temperature conditions: $\mathrm{A}$ : $E G C G$ at $\mathrm{pH} 9$ and $4{ }^{\circ} \mathrm{C}$; B: GCG at pH 9 and $4{ }^{\circ} \mathrm{C}$; C: EGCG at $p H 9$ and $100^{\circ} \mathrm{C}$; D: GCG at $p H 9$ and $100^{\circ} \mathrm{C}$; E: EGCG at $p H 3$ and $100^{\circ} \mathrm{C}$ and F: GCG at pH 3 and $100^{\circ} \mathrm{C}$. 


\subsubsection{EGCG degradation}

The residual profiles of EGCG and EGC in electrospun zein fibers, as well as the unencapsulated control samples are compared in Figure 23. As shown, higher temperature and $\mathrm{pH}\left(100{ }^{\circ} \mathrm{C}, \mathrm{pH}\right.$ 9) increased the rates of EGCG degradation, while low temperature and low $\mathrm{pH}\left(4{ }^{\circ} \mathrm{C}, \mathrm{pH} 3\right)$ enhanced the stability of EGCG. At the same, by contrast, an increase of EGC was observed. Similar to the GCG formation profiles (Figure 22), within the time frame of the experiment, a continual increase of EGC was observed in zein fibers, while a maximum was observed for the unencapsulated samples (Figure 23B, D and F). The depletion of GCG at greater incubation times for the controls implies that EGC was also susceptible to degradation in the aqueous solution, The fact that EGC continued to accumulate in zein fibers (within the time frame of the experiment) suggested that the electrospun zein fibers not only reduced the rate of degradation of EGCG but also protected EGC from further degradation under the experimental conditions. 

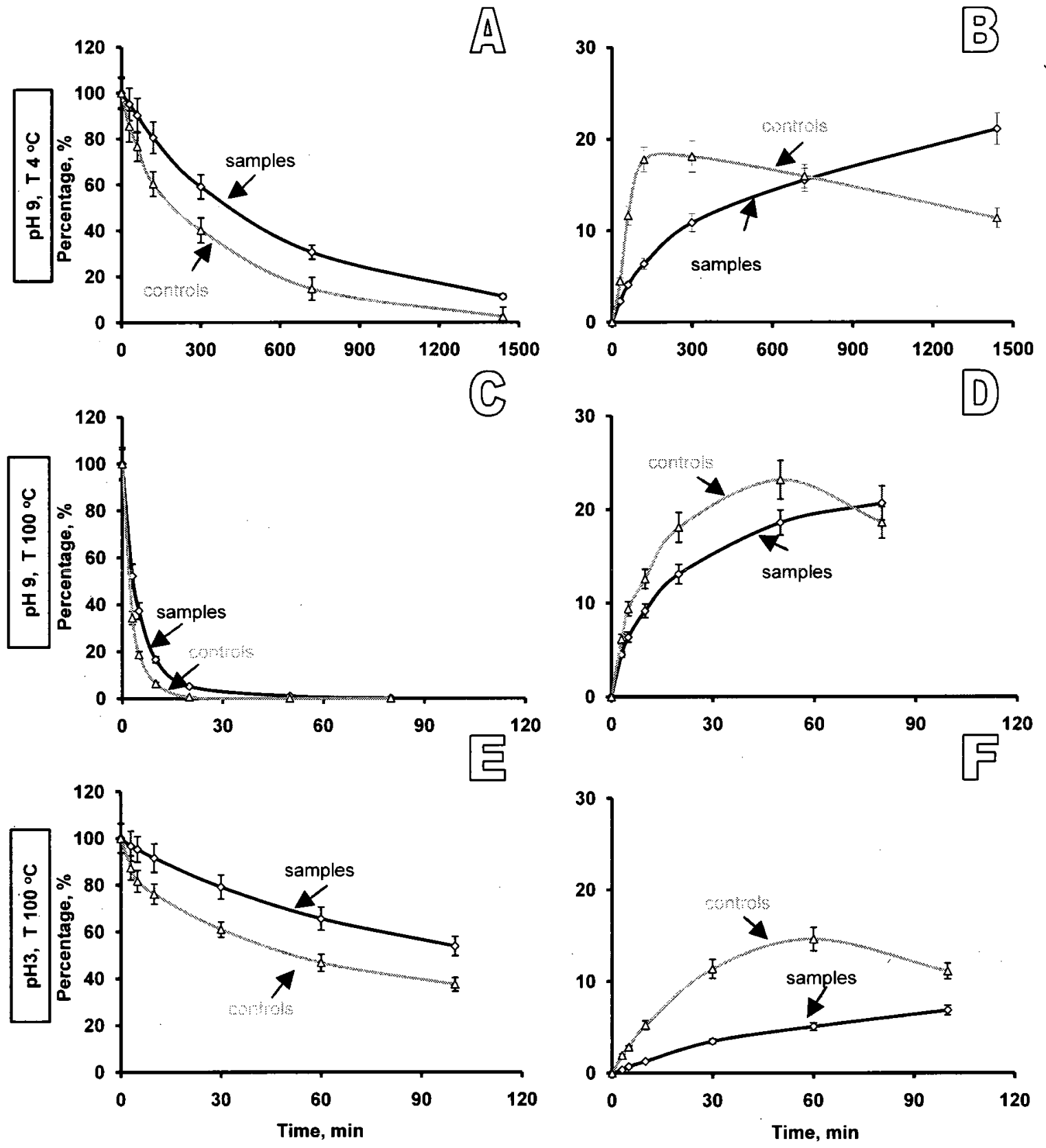

Figure 23 - Percent remaining of residual EGCG encapsulated by electrospun zein fibers and its degraded compounds, EGC, compared to the percentage of EGCG control and its degraded compounds in various conditions. A: EGCG under $\mathrm{pH} 9$ at $4^{\circ} \mathrm{C}$; B: EGC under $\mathrm{pH} 9$ at $4^{\circ} \mathrm{C}$; C: EGCG under $\mathrm{pH} 9$ at $100^{\circ} \mathrm{C}$; D: EGC under $p H 9$ at $100^{\circ} \mathrm{C}$; E: EGCG under $p H 3$ at $100^{\circ} \mathrm{C}$ and F: EGC under $\mathrm{pH} 3$ at $100^{\circ} \mathrm{C}$. 


\subsubsection{Diffusion of EGCG into the aqueous solution}

Figure 24 shows the percent profiles of residual EGCG in fibers and EGCG diffused into the aqueous solution with $\mathrm{pH} 7$ at 60 and $100{ }^{\circ} \mathrm{C}$. As shown in Figure 24 and Figure 25, the concentrations of encapsulated EGCG decreased with increasing time, while the amount of EGCG diffused from the fibers increased with time to a maximum concentration and then declined with time in the aqueous solutions. Moreover, the times when the maximal EGCG occurred were temperature dependent; increasing temperature caused the maximum EGCG concentration to take place sooner; the EGCG released reached a maximum concentration of $28.96 \%$ at $10 \mathrm{~min}$ and then decreased to $8.73 \%$ at 1 hour for $\mathrm{pH} 7$ solution at $100^{\circ} \mathrm{C}$. By contrast, at lower treatment temperature of $60{ }^{\circ} \mathrm{C}, 23.95 \%$ of diffused EGCG was detected at $30 \mathrm{~min}$ and then degraded to $15.20 \%$. This implies that the depletion of EGCG in the fiber can be attributed to at least two mechanisms. One mechanism was related to the degradation while EGCG was immobilized in the fiber, and the second mechanism was caused by the leaching of EGCG into the aqueous solution. The higher temperature employed not only enhanced the diffusion of EGCG into the solution, but also speeded up the degradation of the unprotected EGCG. The increased diffusion and rate of degradation for EGCG at elevated temperature may be a result of disruption of hydrogen bonds acting between zein polymer and EGCG molecules. 

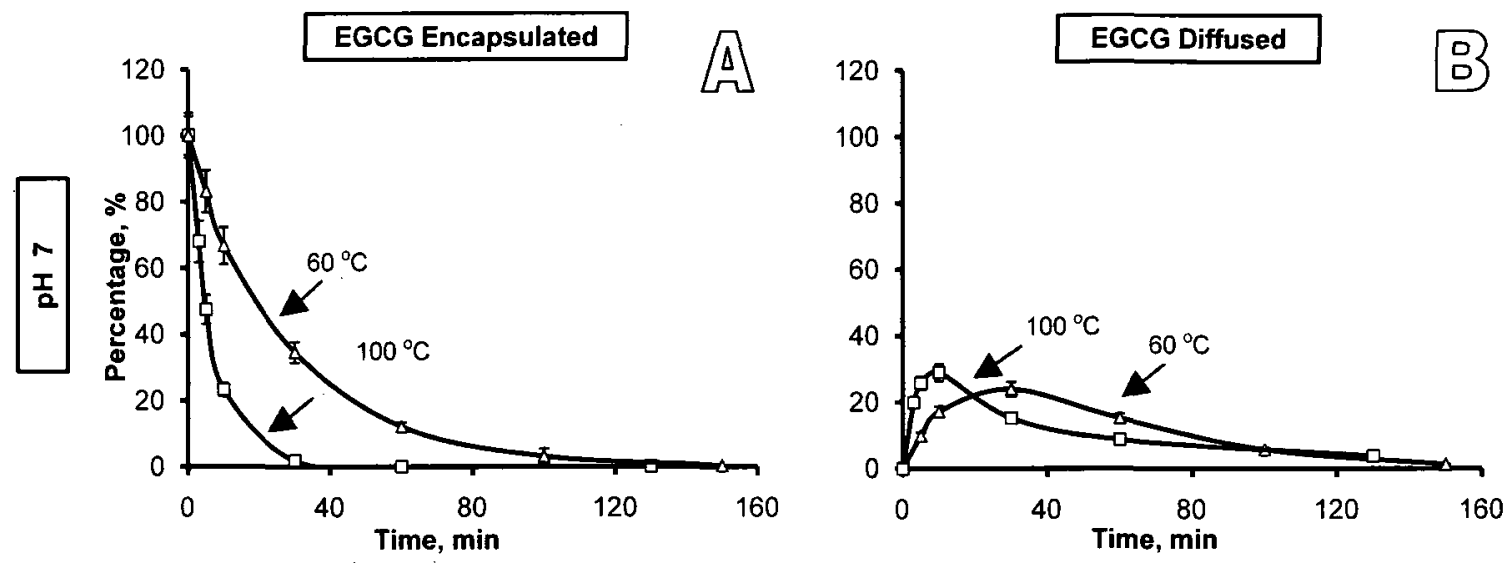

Figure 24 - The percentage of residual EGCG encapsulated by electrospun zein fibers (A) and EGCG diffused from fibers into the aqueous solution (B) at $\mathrm{pH} 7$ at two temperatures.

The effects of $\mathrm{pH}$ on the residual EGCG in zein fibers and EGCG diffused into the aqueous solutions at $60{ }^{\circ} \mathrm{C}$ were illustrated in Figure 25. At $\mathrm{pH} 9$, the concentrations of encapsulated EGCG decreased with increasing time, while EGCG in the solution increased to a maximum concentration and then declined with increasing time. However, at pH 5, the EGCG diffused into the solution appeared to plateau off with increasing time, suggesting that EGCG was more stable under the acidic conditions. Based on the observed greater rate of EGCG release from the fiber at $\mathrm{pH} 9$ than $\mathrm{pH} 5$ (as depicted by the greater initial slope of the concentration profiles for $\mathrm{pH} 9$ ), we can conclude that the release of EGCG from the electrospun zein fibers was $\mathrm{pH}$-dependent. 

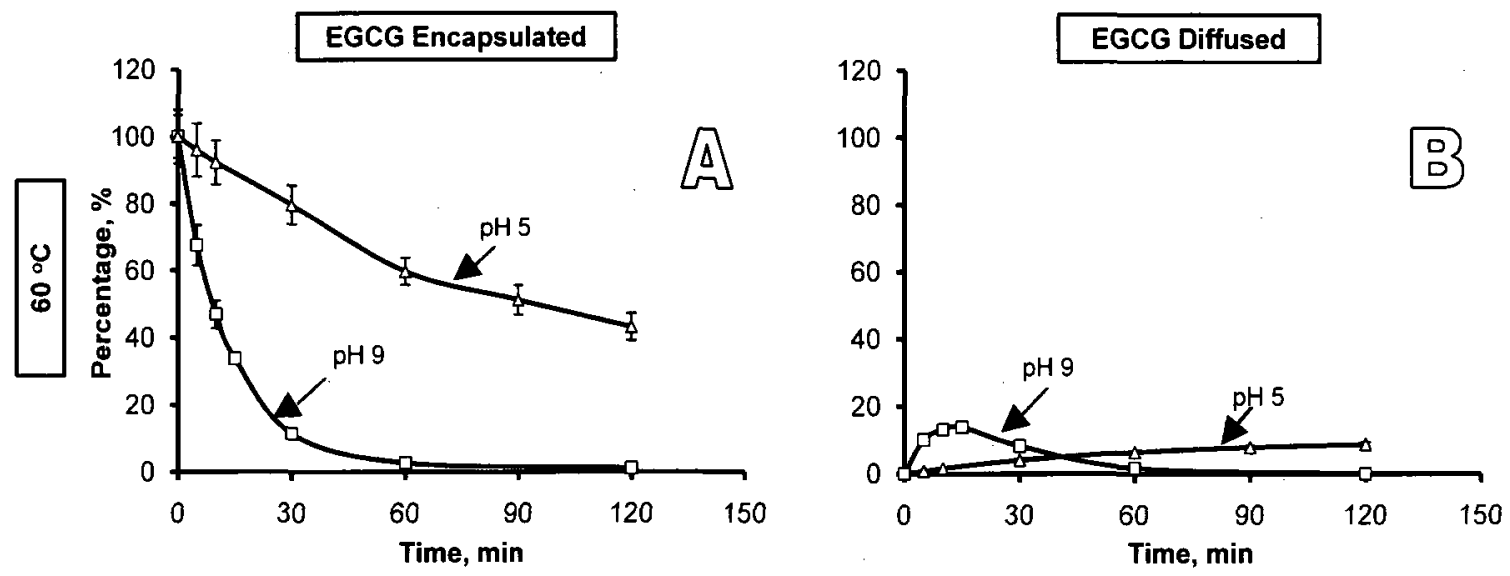

Figure 25 - The percentage of residual EGCG encapsulated by electrospun zein fibers (A) and diffused EGCG from fibers $(B)$ at $60^{\circ} \mathrm{C}$ in aqueous system with $\mathrm{pH}$ 5 and 9. 


\subsection{Conclusions}

In this study, the stability of EGCG encapsulated in electrospun zein fiber and EGCG degraded products were evaluated by applying the elution gradient in HPLC. In conclusion, the stability of zein fiber-encapsulated EGCG was enhanced as compared to the unprotected controls. Based on the experimental observations, the main degradation of EGCG encapsulated in fibers were epimerization from EGCG to GCG, degradation to degraded compounds and diffusion loss from fibers into the aqueous solutions. The encapsulated EGCG samples were vulnerable to alkaline $\mathrm{pH}$ and elevated temperature conditions. The degradation of EGCG encapsulated in electrospun zein fibers was found to increase with increasing $\mathrm{pH}$. Moreover, the higher the temperature, the greater the rate of degradation reaction. Furthermore, it was observed that epimerization and degradation reactions took places simultaneously in the aqueous system, and the stability of EGCG encapsulated in electrospun fibers in aqueous solutions was strongly dependent on $\mathrm{pH}$ and temperature.

Although the stability of EGCG encapsulated in electrospun zein fibers as carriers in aqueous systems has been shown significantly improved, to further investigate this research, kinetic model and rate constants of reaction kinetics are needed. 


\section{KINETIC MODELLING OF EGCG DEGRADATION}

\subsection{Introduction}

In Chapter 6, we observed that the stability of EGCG in electrospun zein fibers was affected by temperature and $\mathrm{pH}$. In this section, a mathematical model was developed to predict the epimerization and degradation kinetics of EGCG under different $\mathrm{pH}$ and temperature conditions that are commonly applied to food products.

It is well documented that epimerization and degradation due to loss of the gallate group are the main reactions that contribute to the degradation of EGCG. Moreover, the epimerization and degradation followed pseudo first-order reactions and that the relationship of rate constants to temperature for these reactions followed the Arrhenius equation (Zimeri and Tong 1999; Wang and others 2006; Wang and others 2008). Wang and other researchers reported that the degradation and epimerization of tea catechins can take place simultaneously during thermal processing (Wang and others 2006). Moreover, the degradation of tea catechins was found to follow first order kinetics (Zimeri and Tong 1999). The temperature- and $\mathrm{pH}$-dependent relationships of rate constant were adequately modeled by the Arrhenius equation. A mathematical model that relates the rate constant to temperature, $\mathrm{pH}$ was developed and validated. Komatsu and others (1992) studied the effects of $\mathrm{pH}$ and temperature on reaction kinetics of catechins in a green tea infusion (Komatsu and others 1992). They found that the apparent first order reaction proceeded in slightly 
acidic media under sterilization conditions, and the turning point temperature on an Arrhenius plot was observed at $82{ }^{\circ} \mathrm{C}$.

Although a number of articles have been published on the degradation kinetics of EGCG, no research has been done to date on modeling the kinetic of degradation for zein fiber-encapsulated EGCG in aqueous solution over a wide temperature range. The objectives of our study were: (1) to develop the preliminary mathematically kinetic model for understanding the degradation of EGCG modified in electrospun zein fibers, (2) to determine the degradation kinetics of EGCG as a function of $\mathrm{pH}$ and temperature in aqueous model solutions, (3) to establish the rate constants for epimerization and degradation reactions, and (4) to calculate and analyze the activation energies of epimerization, degradation and diffusion reactions.

\subsection{Methods and Materials}

The loss of encapsulated EGCG in zein fibers is expected to take place based on three mechanism, namely epimerization to GCG, degradation to nongallate EGC, and diffusion from the fibers into the surrounding aqueous solution. Also, we hypothesize that EGCG undergoes epimerization to GCG. Moreover, both EGCG and GCG could be degraded via the loss of the gallate group during treatments. These models of EGCG degradation are shown schematically in Figure 26. In this representation, the rate constant for the epimerization of EGCG to GCG non-epimer was represented by $k_{1}, k_{2}$ is the rate constant for the diffusion of EGCG from fibers into the aqueous solution and $k_{3}$ represented the 
rate constant of EGCG degradation in the solution. Since the chemical structures of EGCG and GCG only differ slightly between $2 R, 3 R(2,3-c i s$, epi-structure) and $2 S, 3 R(2,3$-trans, nonepi-structure) (Figure 17), it is assumed that the rate constants of degradation of EGCG and GCG structures were similar. Here, $k_{y i}$ represented the degradation rate constant for both GCG and EGCG within the fibers, while $k_{y_{0}}$ is the rate constant of degradation of both GCG and EGCG in the solution. In this model, the assumptions are the reactions are only degradation and epimerization of EGCG; the reactions are one-directional; the diffusion of degraded products are not considered due to no or little amount.

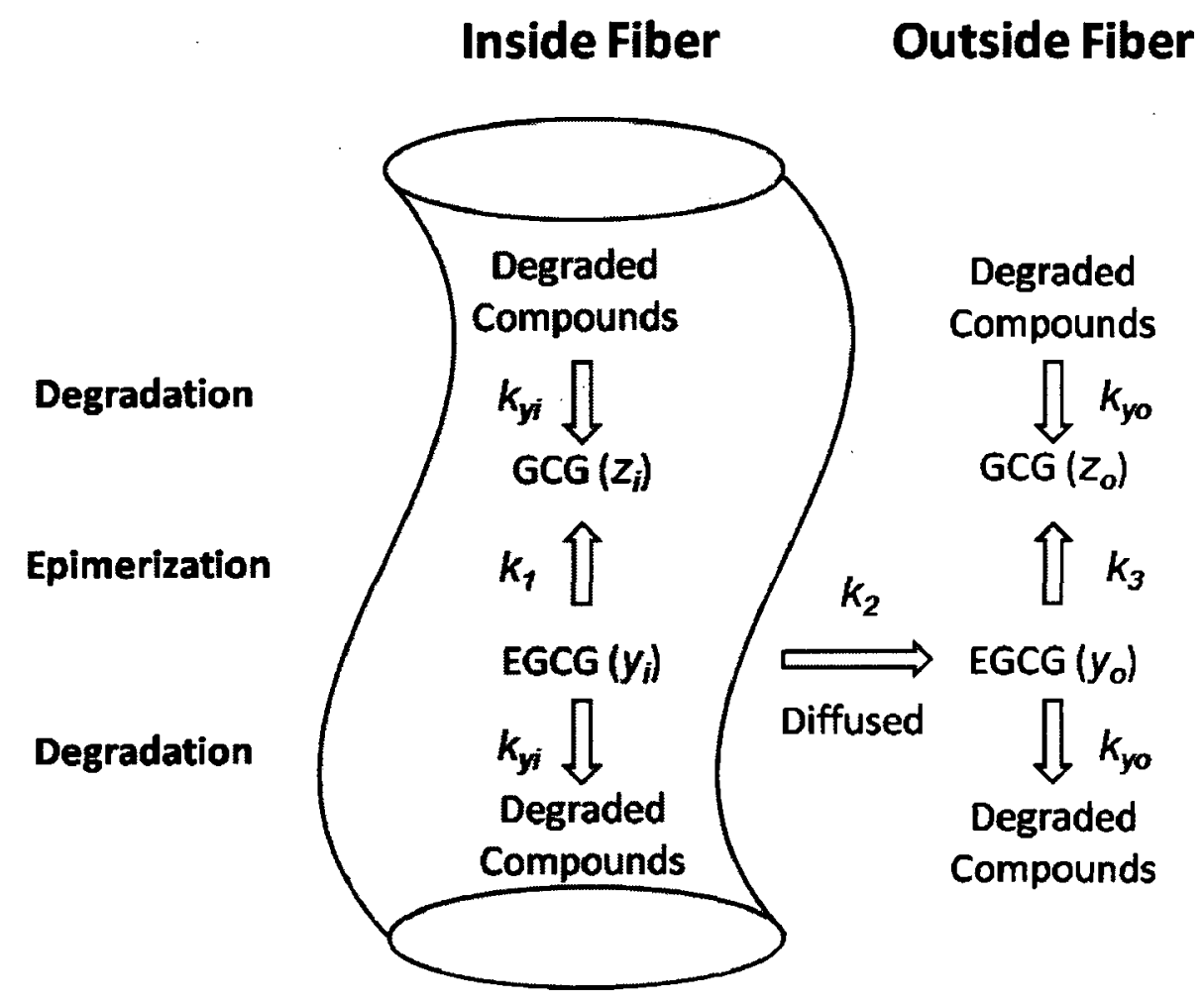

Figure 26 - Schematic representation of EGCG reactions. 


\subsubsection{Modeling Approach}

Since the degradation of EGCG followed first-order kinetics (Zimeri and Tong 1999; Wang and others 2006), the reaction can be described by the following equation:

$$
\ln \left(x / x_{0}\right)=-k t
$$

where $x$ is the concentration of EGCG at time $t, x_{0}$ is the initial concentration, $k$ is the rate constant of EGCG degradation or epimerization. Based on the assumption mentioned above, the rate constants $k$ of the degradation of the total EGCG and GCG could be obtained from the slope of a best-fitted straight line plotted $\ln \left(x / x_{0}\right)$ against time $t$. It was assumed that the rate constants $k$ follow the Arrhenius equation as described below,

$$
k=A \exp \left(-E_{a} / R T\right)
$$

Where $A$ is the frequency factor, $E_{a}$ is the activation energy, $R$ is the ideal gas constant, and $T$ is the temperature in Kelvin. $E_{a}$ and $A$ could be determined from the slope and intercept of the best-fitted straight line of $\ln (k)$ versus $1 / T$ plot.

In our kinetic modeling, both epimerization and degradation were taken into account, because they occurred simultaneously (Wang and others 2006). Assuming the degradation reactions follow pseudo first order kinetics, the reactions can be described as follows: 


$$
\begin{gathered}
y_{i}{ }^{\prime}=-\left(k_{1}+k_{2}+k_{y i}\right) y_{i} \\
z_{i}{ }^{\prime}=k_{1} y_{i}-k_{y o} z_{i} \\
y_{o}{ }^{\prime}=-\left(k_{3}+k_{y o}\right) y_{o}+k_{2} y_{i} \\
z_{o}{ }^{\prime}=k_{3} y_{o}-k_{y o} z_{o}
\end{gathered}
$$

where $y_{i}{ }^{\prime}, z_{i}{ }^{\prime}, y_{o}{ }^{\prime}$ and $z_{o}{ }^{\prime}$ were the rate of change for $y_{i}$ (concentration of EGCG encapsulated in zein fibers), $z_{i}$ (concentration of GCG produced that was immobilized within the fibers), $y_{o}$ (concentration of EGCG diffused from fibers into the solution) and $z_{o}$ (concentration of GCG produced from the EGCG diffused into the solution), respectively.

$$
y_{i}{ }^{\prime \prime}=-\left(k_{1}+k_{2}+k_{y i}\right) y_{i}{ }^{\prime}
$$

rearranging equation 7.7 to

$$
y_{i}^{\prime \prime}+\left(k_{1}+k_{2}+k_{y i}\right) y_{i}^{\prime}=0
$$

Those are second-order ordinary differential equations derived from equation 7.3.

Set $r=\frac{d_{y t}}{d_{t}}=y_{i}{ }^{\prime}$, the formulas above lead to

$$
r \frac{d y_{i}}{d t}+\left(k_{1}+k_{2}+k_{y i}\right) \times r=0
$$


therefore, $r_{1}=-\left(k_{1}+k_{2}+k_{y i}\right), r_{2}=0$. Its solution (Spivak 2006) can be described as:

$$
\begin{gathered}
y_{i}=C_{1} \exp \left(r_{1} t\right)+C_{2} \exp \left(r_{2} t\right) \\
y_{i}{ }^{\prime}=C_{1} r_{1} \exp \left(r_{1} t\right)+C_{2} r_{2} \exp \left(r_{2} t\right)
\end{gathered}
$$

where $C_{1}$ and $C_{2}$ were the rate constants to be determined by the initial conditions. Note that when $t=0, y_{i}(0)=100, z_{i}(0)=0, y_{o}(0)=0$ and $z_{o}(0)=0$. Substituting these into equation 7.10 gives:

$$
\begin{aligned}
& C_{1}+C_{2}=100 \\
& C_{2}=100-C_{1}
\end{aligned}
$$

substituting the above into equation 7.3 and 7.10 and taking $t=0$, the two constants can be derived as:

$$
C_{1}=100, C_{2}=0
$$

substituting $r_{l}$ and $C_{l}$ into equation 7.4, a mathematical model for predicting the concentration of EGCG encapsulated in zein fibers is obtained:

$$
y_{i}=100 \exp \left(-\left(k_{1}+k_{2}+k_{y i}\right) t\right)
$$


Similarly, the model equations for the concentration of EGCG diffused from fibers into the solutions $\left(y_{o}\right)$ and concentration of GCG produced from encapsulated EGCG in fibers (Zimeri and Tong) can be derived as Equations 7.15 and 7.16 , respectively:

$$
\begin{aligned}
& y_{o}=\frac{100 k_{2}}{\left(k_{2}+k_{y i}\right)-\left(k_{1}+k_{2}+k_{y i}\right)}\left[\exp \left(-\left(k_{1}+k_{2}+k_{y i}\right) t\right)-\exp \left(-\left(k_{2}+k_{y i}\right) t\right)\right] \\
& \text { (Equation 7.15) } \\
& z_{i}=\frac{100 k_{1}}{\left(k_{1}+k_{2}\right)}\left[\exp \left(-k_{y i}\right) t-\exp \left(-\left(k_{1}+k_{2}+k_{y i}\right) t\right)\right] \\
& \text { (Equation 7.16) }
\end{aligned}
$$

To determine the specific rate constants of $k_{1}, k_{2}$ and $k_{y i}$, the Matlab programming output is decribed in Appendix 10.4 section.

\subsubsection{Fitting of kinetic models to experiment data}

Matlab 7.0 Non-linear regression analyses were conducted using Matlab 7.0 (The MathWorks Inc., Natick, Ma., U.S.A.) to model the degradation reaction kinetics and estimate the rate constants for epimerization $\left(k_{1}\right)$, degradation $\left(k_{y i}\right)$ and diffusion $\left(k_{2}\right)$ of EGCG encapsulated in fibers. The detailed procedure for the model fitting and rate constants determination for treatments in aqueous solution with $\mathrm{pH} 7$ at $100{ }^{\circ} \mathrm{C}$ is given as an example in Appendix 10.4 section. Microsoft Excel program applied linear regression analysis for first-order degradation of EGCG encapsulated in electrospun zein fibers at various temperatures in 
aqueous solutions, where the goodness of fit was evaluated by the coefficient of determination $\left(R^{2}\right)$.

\subsection{Results and Discussions}

\subsubsection{Verification of the pseudo first-order kinetics for the degradation of EGCG encapsulated in fibers}

According to equation 7.1 , the rate constants $\left(k_{1}+k_{2}+k_{y i}\right)$ of encapsulated EGCG degradation at different temperatures can be obtained by plotting $\ln \left(x / x_{0}\right)$ vs $t$, where $x$ is the total concentration of EGCG determined from fibers and $x_{0}$ is the amount of EGCG added into the formulation. Thus, straight lines are shown in Figure 27. It was found that rate constant $k$ of EGCG degradation increased with increasing of temperature. High values of coefficients of determination $\left(R^{2}\right.$, $0.8531-0.992)$ were obtained for all regressions indicating the assumption that degradation of EGCG at temperatures form $4-100{ }^{\circ} \mathrm{C}$ indeed followed the firstorder kinetics. 


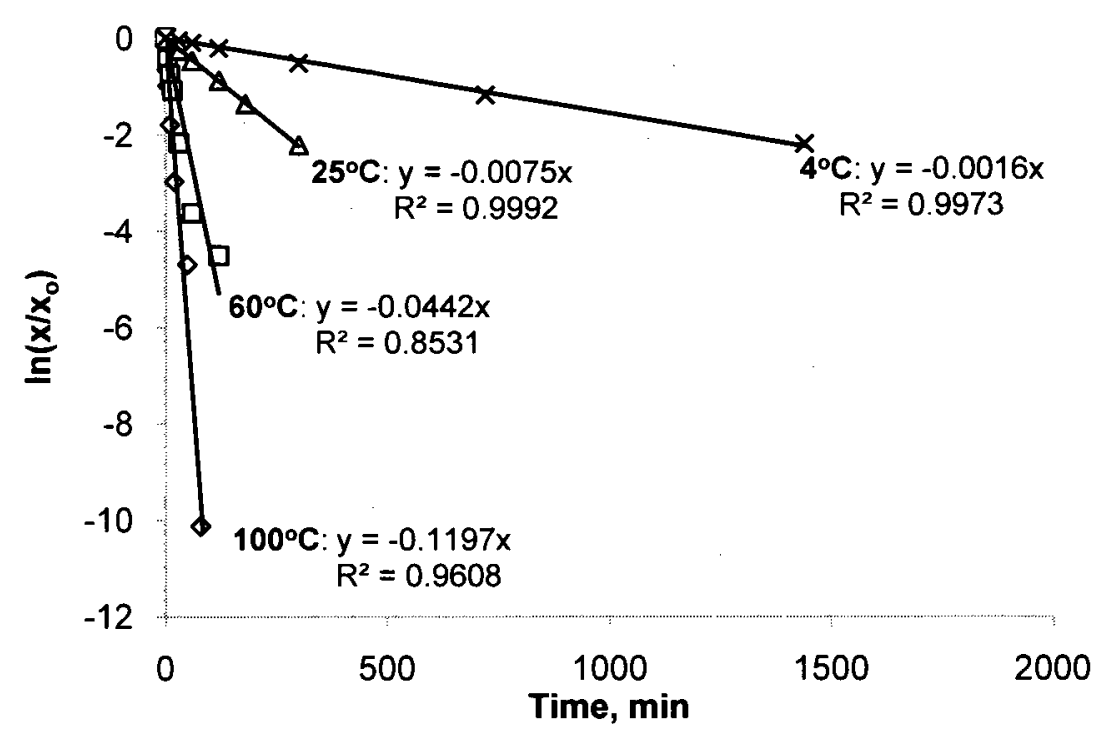

Figure 27 - Arrhenius plots of apparent first-order degradation of EGCG encapsulated in electrospun zein fibers at various temperatures in aqueous solutions with $\mathrm{pH} 9$.

\subsubsection{Rate constant of EGCG epimerization}

Figure 28 shows the Arrhenius plots for $k_{1}$ in aqueous solution under different $\mathrm{pH}$ conditions, as compared to the EGCG controls. These plots showed that the epimerization reaction was enhanced with increasing temperature and $\mathrm{pH}$. The best-fit lines of samples encapsulated in fibers and controls with relatively high values of $R^{2}$ in the range of $0.9799-0.9948$, and the values of RMSE are relatively low in the range of 0.85 to 2.51 , suggesting that activation energy indeed remains unchanged. The epimerization of EGCG encapsulated in fibers and unprotected control both follow the first-order kinetics at various temperatures, the results are in agreement with the report of Wang and others, but they only report on the epimerization of EGCG (unprotected controls) (Wang and others 2008). It is noteworthy that $k_{1}$ values for encapsulated EGCG for $\mathrm{pH} 3$ 
and 5 at $4{ }^{\circ} \mathrm{C}$ are not reported in Figure 28 because no or negligible amounts of GCG were detected. This suggests that EGCG encapsulated in electrospun fibers are stable in acidic aqueous solution and lower temperature. Moreover, the slopes for the control sample plots are larger than those of the encapsulated EGCG plots, implying that the epimerization reaction was slower in the encapsulated samples than the controls. This indicated that the electrospun fibers improved the stability of EGCG in the aqueous systems, which was consistent with the discussion in previous section.

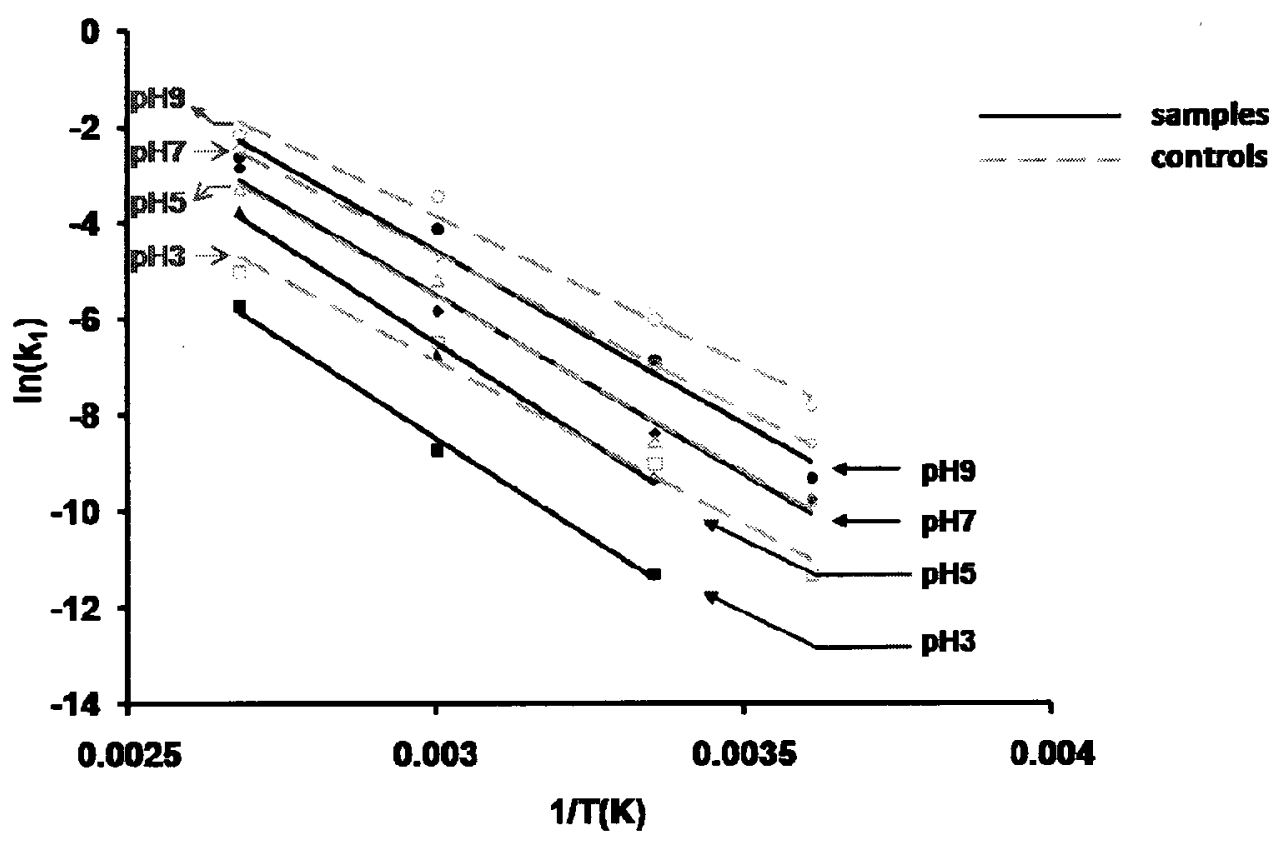

Figure 28 - Arrhenius plots of the rate constants $\left(k_{1}\right)$ for epimerization reactions of EGCG immobilized in electrospun fibers in aqueous system under different $\mathrm{pH}$ conditions. 


\subsubsection{Rate constant of EGCG degradation}

The Arrhenius plots in Figure 29 describe that degradation reactions of EGCG and GCG enhanced with increasing temperature and $\mathrm{pH}$. The slopes for degradation plots of encapsulated EGCG are less than EGCG controls, implying degradation reaction is faster in the controls than in the encapsulated samples. As shown, the values of rate constant $k_{y}$ for encapsulated EGCG and GCG (samples) degradation in solution with $\mathrm{pH} 3$ and 5 at $4{ }^{\circ} \mathrm{C}$ are not described in the figure 29 , possibly because no or little amounts of corresponding degraded compounds detected, suggesting that EGCG encapsulated in electrospun fibers are stable in the acidic aqueous solution at lower temperature. Moreover, the best-fit lines of samples with relatively high values of $R^{2}$ in the range of $0.9714-$ 0.9866 , and the values of RMSE are relatively low in the range of 0.85 to 2.51 , suggesting degradation of EGCG and GCG immobilized in electrospun fibers in the aqueous model solutions followed the pseudo-first order kinetics. Again, the observations provided evidence that the electrospun fibers improved the stability of EGCG in the aqueous systems. 


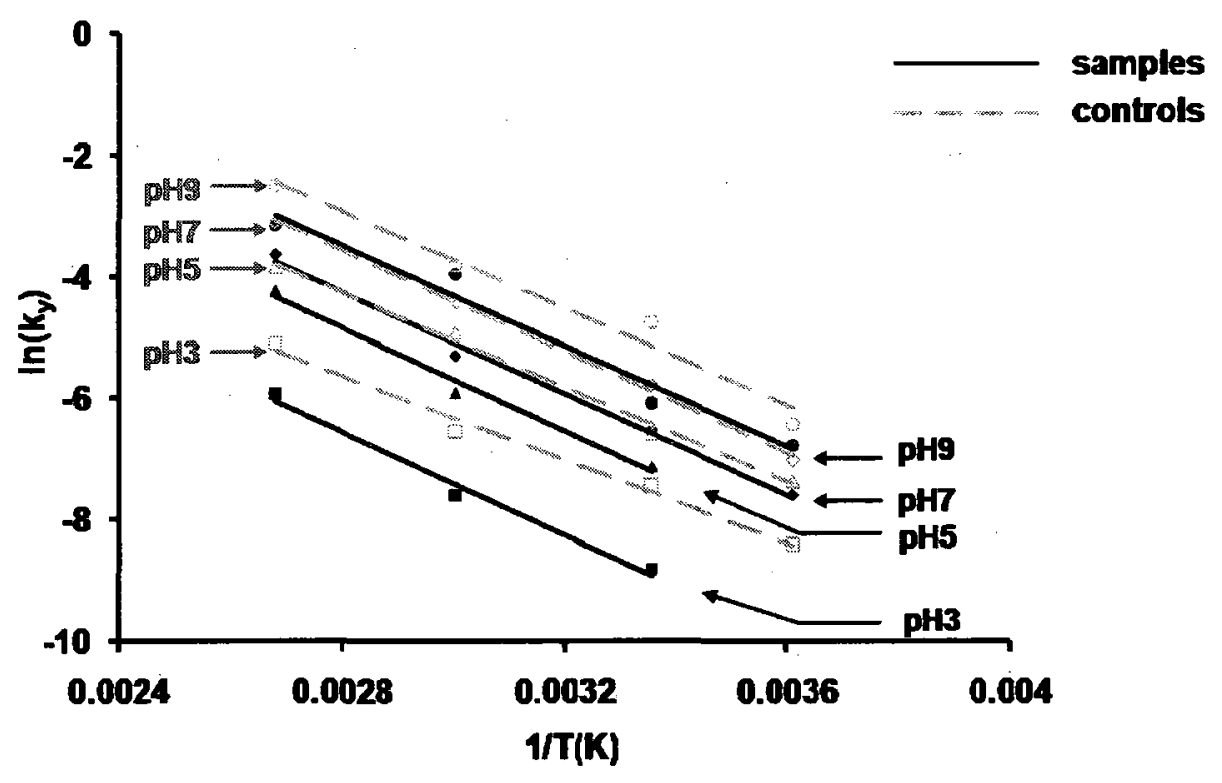

Figure 29 - Arrhenius plots of the rate constants for the degradation reactions of EGCG + GCG immobilized in electrospun fibers in aqueous system under different $\mathrm{pH}$ conditions.

\subsubsection{Rate constant of EGCG release}

Figure 30 describes Arrhenius plots of the rate constants $\left(k_{2}\right)$ of the EGCG diffused from electrospun fibers in aqueous system under different $\mathrm{pH}$ conditions. As shown, higher $\mathrm{pH}$ has higher rate constant of EGCG release from fibers, implying that the release of EGCG from zein fiber is $\mathrm{pH}$-dependent. This interactive effect may be beneficial for promoting the release of EGCG from the fiber in the intestinal condition when the $\mathrm{pH}$ is more alkaline. Moreover, higher temperature influenced the diffusion of encapsulated EGCG from fibers in the solutions, indicating that high temperatures might cause physical damage and the hydrogen bonds between zein fiber and EGCG could be broken during thermal treatments. 


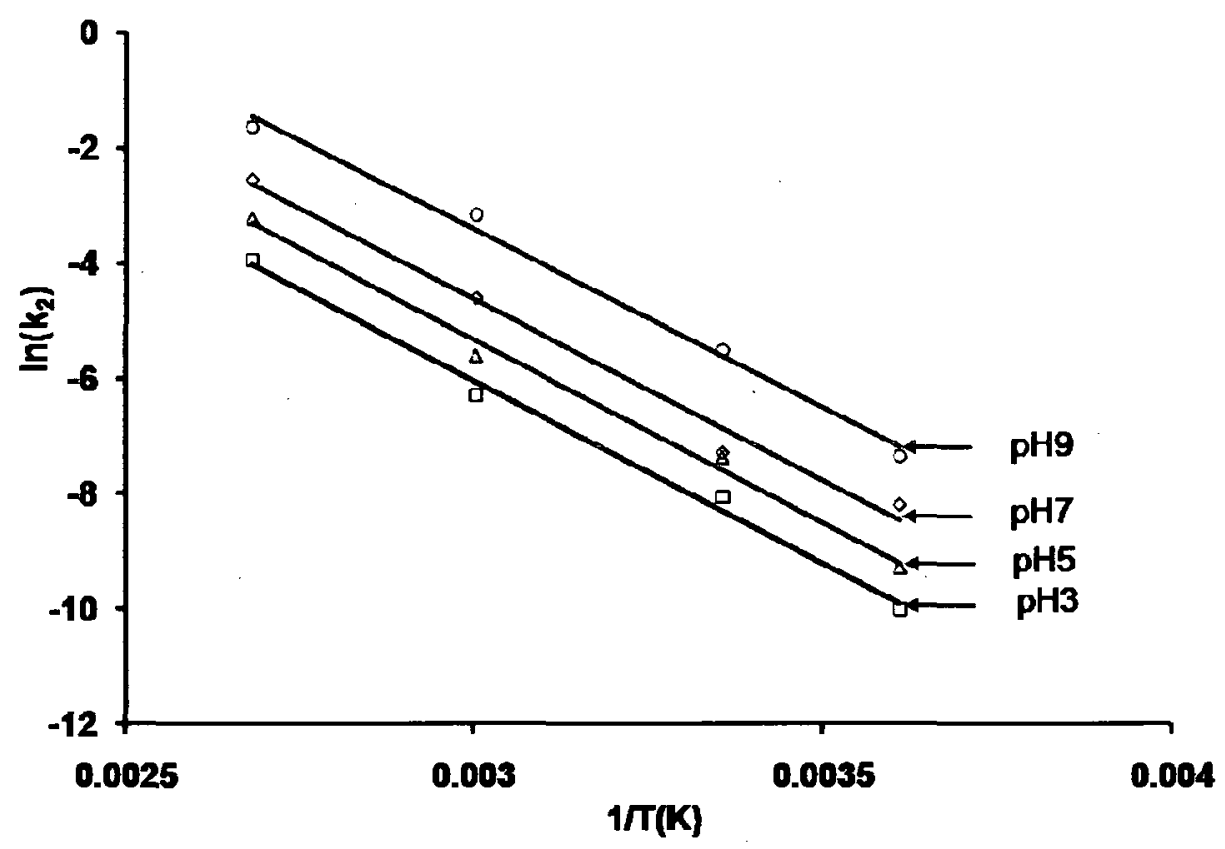

Figure 30 - Arrhenius plots of the rate constants $\left(k_{2}\right)$ of the EGCG diffused from electrospun fibers in aqueous system under different $\mathrm{pH}$ conditions.

\subsubsection{Activation Energy}

Table 2 lists the activation energy (Dousseau and Pezlet 1990) corresponding to the reaction kinetics of EGCG encapsulated in fibers and EGCG controls, computed according to Arrehnius equation. The example for the calsulation procedure is given in the Appendice 10.7 section. The values of $E_{a}$ for the degradation of total (EGCG + GCG), the epimerization from EGCG to GCG, and diffusion from fibers were $61.86,38$ and $52.51 \mathrm{~kJ} / \mathrm{mol}$, respectively. Moreover, for the EGCG controls, the values for activation energy were 54.86 and $32.74 \mathrm{~kJ} / \mathrm{mol}$ for epimerization and degradation of EGCG + GCG respectively. However, these results do not support the report from Wang and others, who stated that the resulting values of $E_{a}$ for the degradation of total 
catechins (EGCG and GCG) and epimerization from EGCG to GCG were 42.78 and $117.59 \mathrm{~kJ} / \mathrm{mol}$ respectively. These differences are possibly due to the fact that the reversible epimerization reaction was considered in their model.

As discussed in Chapter 6, the reaction rates of degradation and epimerization of EGCG was shown to increase with an increase of $\mathrm{pH}$. Here, it was further demonstrated that the rate constants were $\mathrm{pH}$-sensitive while the activation energy of all the reactions remained essentially unchanged with carried compositions in the solutions. For a more definitive conclusion, further research is needed to confirm if similar conclusions could be drawn using a wide temperature range. 
Table 2 - Activation energy (Dousseau and Pezlet) for reaction rate constants of EGCG encapsulated in fibers and EGCG controls in pH $3-9$ aqueous systems at various temperatures, ranged from $4-100^{\circ} \mathrm{C}$.

\begin{tabular}{c|c|c}
\hline Aqueous solution & $\begin{array}{c}\text { Encapsulated sample } \\
\text { in fibers }\end{array}$ & $\begin{array}{c}\text { Unencapsulated } \\
\text { controls }\end{array}$ \\
\hline
\end{tabular}

A: Epimerization from EGCG to GCG.

\begin{tabular}{c|c|c}
\hline $\mathrm{pH} 3$ & $61.34 \mathrm{~kJ} / \mathrm{mol}$ & $54.84 \mathrm{~kJ} / \mathrm{mol}$ \\
\hline $\mathrm{pH} 5$ & $61.34 \mathrm{~kJ} / \mathrm{mol}$ & $54.86 \mathrm{~kJ} / \mathrm{mol}$ \\
\hline $\mathrm{pH} 7$ & $61.86 \mathrm{~kJ} / \mathrm{mol}$ & $54.81 \mathrm{~kJ} / \mathrm{mol}$ \\
\hline $\mathrm{pH} 9$ & $61.71 \mathrm{~kJ} / \mathrm{mol}$ & $54.31 \mathrm{~kJ} / \mathrm{mol}$ \\
\hline
\end{tabular}

B: Degradation of total EGCG and its degraded compounds.

\begin{tabular}{c|c|c}
\hline $\mathrm{pH} 3$ & $38.55 \mathrm{~kJ} / \mathrm{mol}$ & $32.59 \mathrm{~kJ} / \mathrm{mol}$ \\
\hline $\mathrm{pH} 5$ & $38.27 \mathrm{~kJ} / \mathrm{mol}$ & $32.73 \mathrm{~kJ} / \mathrm{mol}$ \\
\hline $\mathrm{pH} 7$ & $38.00 \mathrm{~kJ} / \mathrm{mol}$ & $32.91 \mathrm{~kJ} / \mathrm{mol}$ \\
\hline $\mathrm{pH} 9$ & $37.38 \mathrm{~kJ} / \mathrm{mol}$ & $32.74 \mathrm{~kJ} / \mathrm{mol}$ \\
\hline
\end{tabular}

C: Diffusion of EGCG from fibers.

\begin{tabular}{c|c|c}
\hline $\mathrm{pH} 3$ & $52.22 \mathrm{~kJ} / \mathrm{mol}$ & - \\
\hline $\mathrm{pH} 5$ & $52.38 \mathrm{~kJ} / \mathrm{mol}$ & - \\
\hline $\mathrm{pH} 7$ & $52.51 \mathrm{~kJ} / \mathrm{mol}$ & - \\
\hline $\mathrm{pH} 9$ & $52.57 \mathrm{~kJ} / \mathrm{mol}$ & - \\
\hline
\end{tabular}




\subsubsection{Turning point temperature in the reaction kinetics of EGCG}

Figure 31 combines the Arrhenius plots of the rate constants for epimerization from EGCG to GCG encapsulated in fibers $\left(k_{1}\right.$ indicated as grey line) and degradation of EGCG and GCG ( $k_{y}$ indicated as black line), and also controls are demonstrated as well. Notably, there is a crossing point where the two lines crossed over, indicating that the epimerization and degradation occur simultaneously and the dominance of epimerization and degradation reactions changes with the temperature. As shown, the rates of the reactions alter their order at the crossing points. For instant, there is a crossing point observed at 94 ${ }^{\circ} \mathrm{C}$, as shown in Figure 31A. When the processing temperature is below this point, degradation reaction of EGCG and GCG encapsulated in fibers trends to prevail, the rate constants $k$ for reactions of EGCG in aqueous system with $\mathrm{pH} 3$ can be

levelled as $k_{y}>k_{1}$. When the processing temperature increased to $94{ }^{\circ} \mathrm{C}$ and above, epimerization from EGCG to GCG became a more dominant reaction. Similar trend was observed for the encapsulated EGCG sample, except that the turning point shifted to a lower temperature at $80^{\circ} \mathrm{C}$. The higher the $\mathrm{pH}$ applied to the solution, the lower the temperature where the turning points occurred, indicating that $\mathrm{pH}$ affected the order of EGCG degradation reactions. 

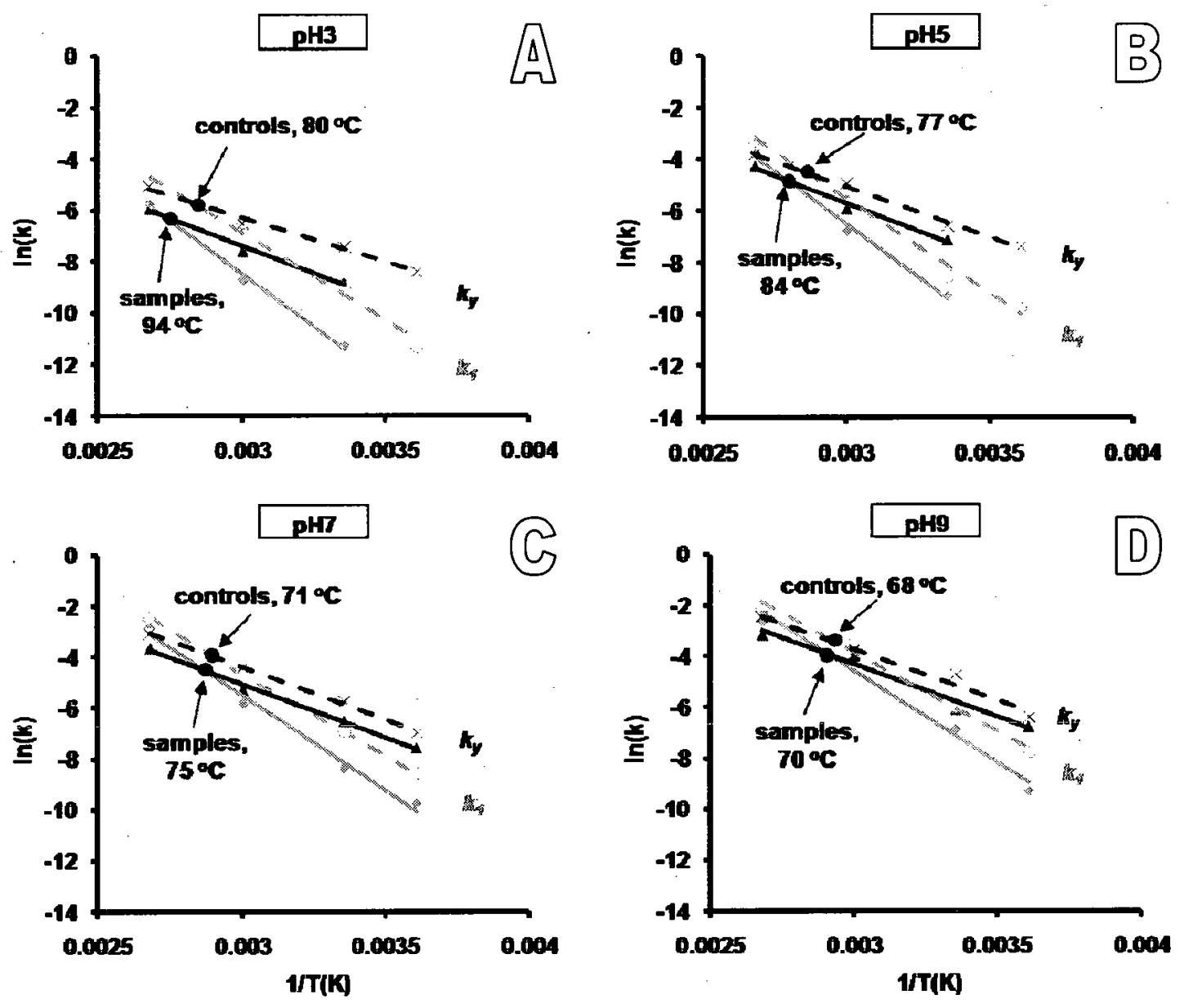

Figure 31 - Arrhenius plots of rate constant $\left(k_{1}\right)$ for the epimerization of from EGCG to GCG in pH 3 - 9 aqueous systems at various temperatures, ranged from $4-100^{\circ} \mathrm{C}$.

\subsubsection{Predictive data}

Figure 32 shows the predicted (solid lines) and experimental data (symbols) of EGCG encapsulated in zein fibers under different temperature and $\mathrm{pH}$ conditions. The RMSE between the modeled and experimental data ranged from 0.85 to 2.51 , and the coefficients of determination $R^{2}$ were all above 0.95 indicating that the model described the experimental results well. The developed 
kinetic models showed good agreement between the experimental results, indicating that within the experimental conditions presented in the present study, the derived models described the degradation kinetics of EGCG quite well.
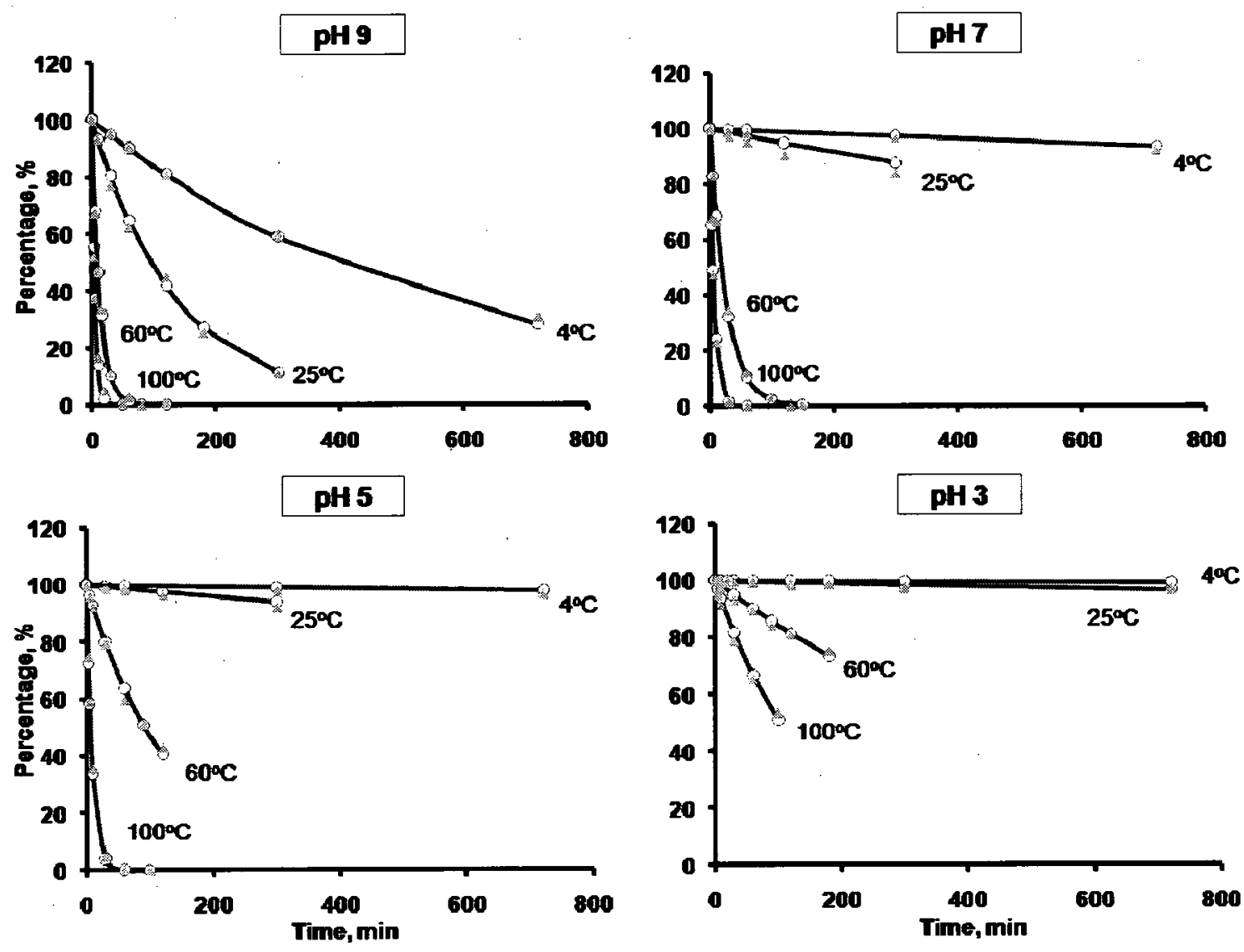

Figure 32 - Modeled and experimental values of residual EGCG in various aqueous systems. RMSE ranged from 0.85 to 2.51 and $R^{2}>0.95$. 


\subsection{Conclusions}

In conclusion, the stability of EGCG in aqueous system was successfully modeled, covering a wide range of temperature from 4 to $100{ }^{\circ} \mathrm{C}$. The mathematical models for predicting the concentration of EGCG during processing were developed. The modeled values fitted the experimental results well. Epimerization and degradation of EGCG followed pseudo-first order kinetics and the rate constants for the reaction kinetics were temperature and $\mathrm{pH}$ dependent. Moreover, higher $\mathrm{pH}$ and temperature caused a higher rate of EGCG release from fibers, implying that the release of EGCG from zein fiber is $\mathrm{pH}$-dependent. This interactive effect may be beneficial for promoting the release of EGCG from the fiber in the intestinal condition when the $\mathrm{pH}$ is more alkaline. It is worth noting that the turning points were identified when the reaction lines crossed over, indicating that the epimerization and degradation occurred simultaneously and the dominance of epimerization and degradation reactions changes with the temperature. The reaction rates altered their order at the crossing points. As the processing temperature was below this point, degradation reaction of EGCG and GCG encapsulated in fibers trended to prevail; When above this temperature, the epimerization reaction could be more profound compared to other reactions. Higher $\mathrm{pH}$ applied to solution, the lower turning points occurred, indicating that $\mathrm{pH}$ affected the order of EGCG degradation reactions. Based on the turning points, it is possible to manipulate the reaction rates of the epimerization from EGCG by adjusting the temperature of a processing so that a desired treatment applied to the product. 
The developed kinetic models showed good agreement between the models predicted values and the experimental results under all experimental conditions. The results with high values of $R^{2}$ and low values of RMSE suggested that the models were valid and of high quality. Therefore, the methodology and results of this study may provide a useful method for future research in assessing the stability of EGCG encapsulated in electrospun zein fibers during various processing, particularly involving with food product. 


\section{CONCLUSIONS AND FUTURE WORKS}

This study developed a method to electrospin zein protein fibers and investigated the interaction between protein structure and EGCG, aimed at enhancing the stability of EGCG in aqueous model systems.

To optimize the electrospinning process, an inverted spinneret setup was adopted for continuous electrospinning of zein ultrafine fiber. The morphology of the resulting fibers was mainly affected by the aqueous ethanol concentration, zein protein concentration, and to a lesser extent, the electric field strength tested. The optimal condition for forming beadless fibers was found to be $20 \%$ protein, $70 \%$ alcohol, and $15 \mathrm{kV}$.

Chapter 5 summarized that the zein fibers resisted solubilization in water, although swelling and plasticization were apparent after the water treatment. The efficacy of zein fibers was tested for stabilization of a green tea polyphenol, (-)epigallocatechin gallate (EGCG), by incorporating the EGCG in zein fiber-forming solutions: Both relative humidity and aging time after spinning were important in determining the stability of EGCG upon subsequent contact with water. Freshly spun fibers were less effective at immobilizing the EGCG upon immersion in water ( $82 \%$ remaining percentage) as compared to fibers that were aged at $0 \%$ relative humidity for at least $1 \mathrm{~d}$ (>98\% remaining percentage) before water immersion. FTIR spectra and curve fitting suggested that hydrogen bonding, hydrophobic interactions, and possibly physical encapsulation are involved in enhancing the retention of EGCG in zein fibers that are immersed in water. More 
studies are currently underway to characterize the stability of EGCG immobilized in zein fibers under various conditions typically encountered in food products.

To further determine the stability of EGCG encapsulated in zein fibers, aqueous solutions at different $\mathrm{pH}$ values $(3-9)$ were chosen as a model system, covering a wide range of temperature from 4 to $100^{\circ} \mathrm{C}$. Compared to the controls, higher amount of remaining EGCG were observed. In general, the stability of EGCG encapsulated in electrospun zein fibers as carriers in aqueous systems was significantly improved. The degradation of EGCG encapsulated in electrospun zein fibers was found to increase with an increase of $\mathrm{pH}$. The higher $\mathrm{pH}$ adopted, the greater the degradation reaction. The alkaline solution with high $\mathrm{pH}$ causes the degradation and deformation. EGCG increased their protonsemiquinone free radicals at neutral or alkaline $\mathrm{pH}$, leading it to be more susceptible to degradation and deformation. However, at $\mathrm{pH} 3$, the stability of EGCG encapsulated in fibers was determined to be high remaining percentage (> $90 \%$ ) after 2 days. The lower the $\mathrm{pH}$ values of the medium, the less the percentage of EGCG degraded. Effect of temperature on stability of EGCG can to be considered as another significant factor in the present study. EGCG degradation was increased with a rise in temperature. The higher temperature adopted, the greater the degradation reaction. The degradation of EGCG encapsulated in fibers is related to epimerization from EGCG to GCG, degradation to related compounds and diffusion from fibers. Furthermore, it was shown th at epimerization and degradation took places simultaneously in the aqueous system at various temperatures. Both reactions of EGCG were 
vulnerable to alkaline $\mathrm{pH}$ and elevated temperature conditions. Diffusion of EGCG from electrospun fibers was influenced by temperature and $\mathrm{pH}$ as well. As a result, the higher the $\mathrm{pH}$ and temperature applied to solutions, the greater the amount of EGCG diffused from the fibers into the test aqueous solutions.

To further investigate this new technology, kinetic model and rate constants of reaction kinetics is necessary. The mathematical models for predicting the concentration of EGCG during processing were developed. The modeled values fitted the experimental results well, epimerization and degradation of EGCG followed pseudo-first order kinetics and the rate constants for the reaction kinetics were temperature and $\mathrm{pH}$ dependent. Moreover, higher $\mathrm{pH}$ and temperature caused the higher rate constant of EGCG release from fibers, implying that the release of EGCG from zein fiber is $\mathrm{pH}$-dependent. This interactive effect may be beneficial for promoting the release of EGCG from the fiber in the intestinal condition when the $\mathrm{pH}$ is more alkaline. Turning points of reaction were identified when the dominance of epimerization and degradation switched with the temperature. The reactive rates of the reactions altered their dominance at the crossing points. When the processing temperature was below this point, degradation reaction of EGCG and GCG encapsulated in fibers trended to prevail; while above this temperature, the epimerization reaction was dominant. The higher the $\mathrm{pH}$ applied to the tested solution, the lower the turning point temperature occurred, indicating that $\mathrm{pH}$ affected the dominance of EGCG degradation reactions. Thus, it is possible to manipulate the reaction rates of the 
epimerization from EGCG by adjusting the temperature of a processing so that a desired treatment applied to the product.

The developed kinetic model showed good agreement between the model predicted values and the experimental results under all experimental conditions. The results with high values of $R^{2}$ and low values of RMSE suggested that the models were valid and of high quality. Therefore, the methodology and results of this study may provide a useful method for future research in assessing the stability of EGCG encapsulated in electrospun zein fibers during various processing, particularly when involved with food products.

Overall, the main goal of stabilizing EGCG by emcapsulating it in zein fibers has been achieved. However, there are still a number of unanswered questions that still need to be explored. The followings are recommendations for further studies:

* This study successfully worked on electropsinning zein protein ultrafine fibers and considering the effects of main spinning factors such as zein concentration, aqueous ethanol concentration and applied DC voltage. However, other factors should be considered as well, such as flow rate of polymer solution, distance between collector and needle tip, temperature and relative humidity. Although there were limited references related to electrospin zein fibers at the time when experiments started, future studies need to consider these factors which are expected to influence the morphology of spun fibers. 
* Based on the stability study in Chapter 5, the optimal condition for forming beadless fibers was found to be $20 \%$ protein, $70 \%$ alcohol, and $15 \mathrm{kV}$. Although this optimal condition could produce beadless fibers, other condition might improve the stability of EGCG as well, such as micro-sized droplets (Figure 5A) and fibers with beads (Figure 7D). It will be necessary to apply these conditions for stability study in the future study.

* According the references related to the amide I and II bands of protein, the position of the $\alpha$-helices and $\beta$-sheets was well described. However, the curve fittings of EGCG and the secondary structure of electrospun zein protein fibers were not studied. In order to improve the results, further study of curve fitting should be done.

* EGCG has strong antioxidative activity in relation to protection against carious diseases, and also this antioxidative activity is structure-dependent (Wang and others 2008). EGCG might undergo complex reactions such as oxidation, polymerization and epimerization during thermal processing. Therefore, oxidation reaction should be considered as one of the degradation reactions for EGCG. However, because the kinetic model was too complicated to solve and no research articles were published for EGCG encapsulated in fibers, future studies in these areas are needed.

* From the predictive model in Figure 26, all the reactions in the present research were determined as irreversible. However, the results of degradation kinetic of EGCG were not in the agreements with the reports 
by Wang and others, possibly due to the reversible epimerization were applied in their model (Wang and others 2008).

* EGCG were susceptible to formation of a semiquinone free radical in alkaline solution, indicating that the free-radical scavenging capacity of EGCG increased with increasing $\mathrm{pH}$ because at neutral or alkaline $\mathrm{pH}$, EGCG increased their protein-donating potential and became easier to form the corresponding semiquinone free radicals (Yoshioka and others 1991). The adjacent hydroxyl group at C-5' on the ring of EGCG was vulnerable, as a result ECG could be formed in the neutral or alkaline $\mathrm{pH}$. Therefore, for the future study, GCG, ECG, CG, EGC and GC should be included as degraded products in the kinetic model.

- Based on the observed data, the electrospun zein fibers are expected to find application in some food products. Further testing in food products is needed to elucidate their efficacy and robustness as an encapsulant. 


\section{EFERENCES}

Akanbi CT, Oludemi FO. 2004. Effect of processing and packaging on the lycopene content of tomato products. Int J Food Prop 7(1):139-52.

Argos P, Pedersen K, Marks M, Larkins B. 1982. A structural model for maize zein proteins. J Biol Chem 257:9984-90.

Arrondo JLR, Goni FM. 1999. Structure and dynamics of membrane proteins as studied by infrared spectroscopy. Progr Biophys Mol Bio 72:367-405.

Asano K, Shinagawa K, Hashimoto N. 1982. Characterization of haze-forming proteins of beer and their roles in chill haze formations. J Am Soc Brew Chem 40:147-54.

Ayutsede J, Gandhi M, Sukigara S, Micklus M, Chen H-E, Ko F. 2005. Regeneration of Bombyx mori silk by electrospinning. Part 3: characterization of electrospun nonwoven mat. Polymer 46(5):1625-34.

Baba S, Osakabe N, Natsume M, Muto Y, Takizawa T, Terao J. 2001. In vivo comparison of the bioavailability of $(+)$-catechin, $(-)$-epicatechin and their mixture in orally administered rats. J Nutr 131:2885-91.

Basu TK, Donaldson D. 2003. Intestinal absorption in health and disease: micronutrients. Best Pract Res Cl Ga 17(6):957-79.

Boland ED, Matthews JA, Pawlowski KJ, Simpson DG, Wnek GE, Bowlin GL. 2004. Electrospinning collagen and elastin: Preliminary vascular tissue engineering. Front Biom 9:1422-32.

Brouwer IA, Van DM, West CE, Steegers-Theunissen RPM. 2001. Bioavailability and bioefficacy of folate and folic acid in man. Nutri Res Rev 14:267-93.

Buttafoco L, Kolkman NG, Engbers-Buijtenhuijs P, Poot AA, Dijkstra PJ, Vermes I, Feijen J. 2006. Electrospinning of collagen and elastin for tissue engineering applications. Biomaterials 27:724-34. 
Cai Y, Anavy ND, Chow H-HS. 2002. Contribution of presystemic hepatic extraction to the low oral bioavailability of green tea catechins in rats. Drug Metab Dispos 30:1246-9.

Catterall F, King LJ, Clifford MN, Loannides C. 2003. Bioavailability of dietary doses of ${ }^{3} \mathrm{H}$-labelled tea antioxidants (+)-catechin and (-)-epicatechin in rat. Xenobiotica 33(77):743-53.

Chen ZY, Zhu QY, Wong YF, Zhang Z, Chung HY. 1998. Stabilizing effect of ascorbic acid on green tea catechins. J Agr Food Chem 46(7):2512-6.

Chen ZY, Chan PT. 1996. Antioxidative activity of green tea catechins in canola oil. Chem Phys Lipids 82(2):163-72.

Chen ZY, Zhu QY, Tsang D, Huang Y. 2001. Degradation of green tea catechins in tea drinks. J Agric Food Chem 49(1):477-82.

Chew SY, Wen Y, Dzeins Y, Leong KW. 2006. The role of electrospinning in the emerging field of nanomedicine. Curr Pharm Des 12(36):4751-70.

Deitzel JM, Kleinmeyer J, Harris D, Beck Tan NC. 2001. The effect of processing variables on the morphology of electrospun nanofibers and textiles. Polymer 42(1):261-72.

Demir MM, Yilgo I, Yilgo E, Erman B. 2002. Electrospinning of polyurethane fibers. Polymer 43(11):3303-9.

Dong J, Sun Q, Wang J-Y. 2004. Basic study of corn protein, zein, as biomaterial in tissue engineering, surface morphology and biocompatibility. Biomaterials 25(19):4691-7.

Dousseau F, Pezlet M. 1990. Determination of the secondary structure content of proteins in aqueous solutions from their amide I and amide II infrared bands, comparison between classical and partial least-square methods. Biochem 29(37):8771-9. 
Fersht A 1999. Structure and mechanism in protein science: a guide to enzyme catalysis and protein folding. New York: W. H. Freeman \& Company. 1-54 p.

Fong H, Liu W, Wang C-S, Vaia RA. 2002. Generation of electrospun fibers of nylon 6 and nylon 6-montmorillonite nanocomposite. Polymer 43(3):77580.

Forato LA, Bicudo TD, Colnago LA. 2003. Conformation of $\alpha$ zeins in solid state by fourier transform IR. Biopolymer 72(6):421-6.

Gianazza E, Eberini I, Arnoldi A, Wait R, Sirtori CR. 2003. A proteomic investigation of isolated soy proteins with variable effects in experimental and clinical studies. J Nutr 133(1):9-14.

Gianazza E, Viglienghi V, Righetti PC, Salamini F. 1977. Amino acid composition of zein molecular components. Phytochemistry 16:315-7.

Gibbs BF, Kermasha S, Alli I, Mulligan C. 1999. Encapsulation in the food industry: a review. Int J Food Sci Nutr 50(3):213-24.

Hammel E, Tang X, Trampert M, Schmitt T, Mauthner K, Eder A, Potschke P. 2004. Carbon nanofibers for composite application. Carbon 42:1153-8.

Haslam E 1989. Plant polyphenols vegetable tannins revisited. New York: Cambridge University Press. 14-89 p.

Hayat MA 1978. Introduction Biological Scanning Electron Microsopy. Maryland: University Park Press.

Higdon JV, Frei B. 2003. Tea catechins and polyphenols: health effects, metabolism, and antioxidant functions. Crit Rev Food Sci Nutr 43(1):89143.

Hoppert M 2003. Microscopy Techniques in Biotechnology: WILEY-VCH Gmbh \& Co. KGaA. 191-210.

Huang L, Nagapudi K, P.Apkarian R, Chaikof E. 2001. Engineered clooagenPEO nanofibers and fabrics. J Biomater Sci Polym Ed 12(9):979-93. 
Huang ZM, Zhang YZ, Kotaki M, Ramakrishna S. 2003. A review on polymer fibers by electrospinning and their applications in nanocomposites. Compos Sci Technol 63:2223-53.

Huang ZM, Zhang YZ, Ramakrishna S, Lim CT. 2004. Electrospinning and mechanical characterization of gelatin nanofibers. Polymer 45:5361-8.

Hurrell RF. 1997. Preventing iron deficiency through food fortification. Nutr Rev 55(6):210-22.

Ismail AA, Mantsch HH, Wong PTT. 1992. Aggregation of chymotrypsinogen: portrait by infrared spectroscopy. Biochim Biophys Acta 1121:183-8.

Jackson M, Mantsch HH. 1995. The use and misuse of FTIR spectroscpoy in the determination of protein structure. Crit Rev Biochem Mol 30(2):95-120.

Jarusuwannapoom T, Hongrojjanawiwat W, Jitjaicham S, Wannatong L, Nithitanakul M, Pattamaprom C, Koombhongse P, Rangkupan R, Supaphol P. 2005. Effect of solvents on electro-spinnability of polystyrene solutions and morphological appearance of resulting electrospun polystyrene fibers. Eur Polym J 41(3):409-21.

Jin HJ, Fridrikh SV, Rutledge GC, Kaplan DL. 2002. Electrospinning Bombyx mori silk with poly(ethylene oxide). Biomacromolecules 3:1233-9.

Jun Z, Hou H, Schaper A, Wendorff JH, Greiner A. 2003. Poly-L-lactide nanofibers by electrospinning-influence of solution viscosity and electrical conductivity on fiber diameter and fiber morphology. e-Polymers nr. 009:1-9.

Jung C. 2000. Review Insight into protein structure and protein-ligand recognition by fourier transform infrared spectroscopy. J Mol Recognit 13:325-51.

Katiyar SK, Ahmad N, Mukhtar H. 2000. Green tea and skin. Arch Dermatol 136(8):989-94. 
Kiatgrajai P, Wellons JD, Gollob L, White JD. 1982. Kinetics of epimerization of $(+)$-catechin and its rearrangement to catechinic acid. J Org Chem 47(15):2910-2.

Ko FK. 2003. Nanofiber technology: bridging the gap between nano andmacro world. In: NATO-Advance Study Institute (ASI) Nanoengineered Nanofibrous Materials; 2003 September 1-12; Antalya, Turkey: Drexel Nanotechnology Inst. 22 .

Komatsu Y, Suematsu S, Hisanobu Y, Saigo H, Matsuda R, Hara K. 1992. Effects of $\mathrm{pH}$ and temperature on reaction kinetics of catechins in green tea infusion. Biosci Biotech Biochem 57(6):907-10.

Krimm S, Bandekar J. 1986. Vibrational spectroscopy and conformation of peptides, polypeptides, and proteins. Adv Protein Chem 38:181-364.

Landis-Piwowar KR, Huo C, Chen D, Milacic V, Shi GQ, Chan TH, Dou QP. 2007. A novel prodrug of the green tea polyphenol (-)-Epigallocatechin-3-Gallate as a potential anticancer agent. Cancer Res 67(9):4303-10.

Li D, Xia Y. 2004. Electrospinning of nanofibers: reinventing the wheel? Adv Mater 16(14):1151-70.

Li Y, Lim L-T, Kakuda Y. 2009. Electrospun zein fibers as carriers to stabilize (-)epigallocatechin gallate. J Food Sci 74(3):233-40.

Madhan B, Subramanian V, Raghava R, Nair BU, Ramasami T. 2005. Stabilization of collagen using plant polyphenol: Role of catechin. Intl J Biol Macromol 35:47-53.

Maiti TK, Ghosh KS, Dasgupta S. 2006. Interaction of (-)-epigallocatechin-3gallate with human serum albumin: fluorescence, fourier transform infaraed, circular dichroism, and docking studies. Protein: Struct Funct Bioinform 64:355-62.

Matthews JA, Wnek GE, Simpson DG, Bowlin GL. 2002. Electrospinning of collagen fibers. Biomacromolecules 3(2):232-8. 
Mckillop DJ, Pentieva K, Daly D, Mcpartlin JM, Hughes J, Strain JJ, Scott JM, Manulty H. 2002. The effect of different cooking methods on folate retention in various foods that are amongst the major contributors to folate intake in the UK diet. Br J Nutr 88:681-8.

Meersman F, Smeller L, Heremans K. 2002. Comparative Fourier transform infrared spectroscopy study of cold-pressure, and heat-induced unfolding and aggregation of myoglobin. Biophyl J 82:2635-44.

Megelski S, Stephens JS, Chase DB, Rabolt JF. 2002. Micro- and nanostructured surface morphology on electrospun polymer fibers. Macromolecules 35(22):8456-66.

Mejia CD, Mauer LJ, Hamaker BR. 2007. Similarities and differences in secondary structure of viscoelastic polymers of maize a-zein and wheat gluten proteins. J Cereal Sci 45:353-9.

Miyoshi T, Toyohara K, Minematsu H. 2005. Preparation of ultrafine fibrous zein membranes via electrospinning. Polym Int 54:1187-90.

Mizutani Y, Matsumura Y, Imamura K, Nakanishi K, Mori T. 2003. Effects of water activity and lipid addition on secondary structure of zein in powder systems. J Agr Food Chem 51:229-35.

Nguyen MT, Indrawati, Hendrickx M. 2003. Model studies on the stability of folic acid and 5-methyltetrahydrofolic acid degradation during thermal treatment in combination with high hydrostatic pressure. J Agric Food Chem 51(11):3352-7.

Nisha P, Singhal RS, Pandit AB. 2005. Degradation kinetics of folic acid in cowpea (Vigna catjang L.) during cooking. Int J Food Sci Nutr 56(6):38997.

Obanda M, Owuor PO, Mang'oka R. 2001. Changes in the chemical and sensory quality parameters of black tea due to variations of fermentation time and temperature. Food Chem 75(4):395-404. 
Outtrup H, Fogh R, Schaumburg K 1987. Proceedings of the 21st Congress of the European Brewery Convention Madrid: Elsevier applied science 583$90 \mathrm{p}$.

Panick G, Malessa R, Winter R. 1999. Differences between the pressure- and temperature-induced denaturation and aggregation of $\beta$-lactoglobulin $A, B$ and $A B$ monitored by FT-IR spectroscopy and small-angle $X$-ray scattering. Biochemistry-US 38:6512-9.

Remondetto GE, Beyssac E, Subirade M. 2004. Iron availability from whey protein hydrogels: an in vitro study. J Agric Food Chem 52:8137-43.

Reneker DH, Yarin AL, Fong H, Koombhonmgse S. 2000. Bending instability of electrically charged liquid jets of polymer solutions in electrospinning. $J$ Appl Phys 87:4531-47.

Ristow KA, Gregory JF, Damron BL. 1982. Thermal processing effects on folacin bioavailability in liquid model food systems, liver, and cabbage. J Agric Food Chem 30(5):801-6.

Salah N, Miller NJ, Paganga G, Tijburg L, Bolwell GP, Riceevans C. 1995. Polyphenolic flavanols as scavengers of aqueous phase radicals and as chain-breaking antioxidants. Archiv Biochem Biophy 322(2):339-46.

Salgueiro MJ, Zubillaga M, Lysionek A, Cara R, Weill R, Boccio J. 2002. Fortification strategies to combat zinc and iron deficiency. Nutr Rev 60(2):52-8.

Sang S, Lee M-J, Hou Z, Ho C-T, Yang C. 2005. Stability of tea polyphenol (-)epigallocatechin-3-gallate and formation of dimers and epimers under common experimental conditions. J Agric Food Chem 53:9478-84.

Selling GW, Sessa DJ. 2007. Sample preparation and testing methods affect the physical properties and evaluation of plasticized zein. Ind Crop Prod:26673.

Seto R, Nakamura H, Nanjo F, Hara Y. 1997. Preparation of epimers of tea catechins by heat treatment. Biosci Biotech Bioch 70(3):337-44. 
Shukla R, Munir C. 2001. Zein: the industrial protein from corn. Ind Crops Prod 13(3):171-92.

Siebert KJ, Troukhanova NV, Lynn PY. 1996. Nature of polyphenol-protein interactions. J Agric Food Chem 44:80-5.

Son WK, Youk JH, Lee TS and Park WH. 2004. The effects of solution properties and polyelectrolyte on electrospinning of ultrafine poly(ethylene oxide) fibers. Polymer 45(9): 2959-66.

Spivak M 2006. Calculus. Cambridge: Cambridge University Press. 670 p.

Stitzel JD, Pawlowski KJ, Wnek GE, Simpson DG, Bowlin GL. 2001. Arterial smooth muscle cell proliferation on a novel biomimicking, biodegradable vascular graft scaffold. J Biomater Appl 16(1):22-33.

Theron S, Zussman E, Yarin AL. 2004. Experimental investigation of the governing parameters in the electrospinning of polymer solutions. Polymer 45:2017-30.

Torres-Giner S, Ocio MJ, Lagaron JM. 2008. Development of active antimicrobial fiber based chitosan polysaccharide nanostructures using electrospinning. Engr Life Sci 8(3):300-14.

Vega-Lugo A-C, Lim L-T (2008a). Electrospinning of soy protein isolate and poly(lactic acid) ultrafine fibers for controlled release of allyl isothiocyanate. Department of Food Science. Guelph, University of Guelph. Mater of Science: 106.

Vega-Lugo A-C, Lim L-T. 2008b. Electrospinning of soy protein isolate nanofibers. J Biobased Mater Bioenergy 2(3):223-30.

Wang H, Provan GJ, Helliwell K. 2003. HPLC determination of catechins in tea leaves and tea extracts using relative response factors. Food Chem 81(2):307-12. 
Wang M, Hsieh AJ, Rutledge GC. 2005. Electrospinning of poly (MMA-co-MAA) copolymers and their layered silicate nanocomposites for improved thermal properties. Polymer 46:3407-18.

Wang R, Zhou W, Wen R-A. 2006. Kinetic study of the thermal stability of tea catechins in aqueous systems using a microwave reactor. J Agric Food Chem 54:5924-32.

Wang R, Zhou W, Jiang X. 2008. Reaction kinetics of degradation and epimerization of epigallocatechin gallate in aqueous system over a wide temperature range. J Agr Food Chem 56:2694-701.

Woerdeman DL, Ye P, Shenoy S, Parnas RS, Wnek GE, Trofimova O. 2005. Electrospun fibers from wheat protein: investigation of the interplay between molecular structure and the fluid dynamics of the electrospinning process. Biomacromolecules 6:707-12.

Xia S, Xu S. 2005. Ferrous sulfate liposomes-preparation, stability and application in fluid milk. J Food Sci 38:289-96.

Yao C, Li X, Song T. 2007. Electrospinning and crosslinking of zein nanofiber mats. J Appl Polym Sci 103:380-5.

Yilmaz Y. 2006. Novel uses of catechins in foods. Trends Food Sci Tech 17(2):64-71.

Yoshioka H, Sugiura K, Kawahara R, Fujita T, Makino M, Kamiya M, Tsuyumu S. 1991. Formation of radicals and chemiluminescence during the autoxidation of tea catechins. Agric Biol Chem 55(11):2717-23.

Yuliani S, Bhandari B, Rutgers R, D' Arcy B. 2004. Application of microencapsulated flavor to extrusion product. Food Rev Int 20(2):163-85.

Yusuf Y. 2006. Novel uses of catechins in foods. Trends Food Tech 17:64-71.

Zaveri NT. 2006. Green tea and its polyphenolic catechins: Medicinal uses in cancer and noncancer applications. Life Sci 78(18):2073-80. 
Zeng J, Xu X, Chen X, Liang Q, Bian X, Yang L, X J. 2003. Biodegradable electrospun fibers for drug delivery. J Control Release 92(3):227-31.

Zhang Y, Ouyang H, Lim CT, Ramakrishna S, Huang Z-M. 2005. Electrospinning of gelatin fibers and gelatin/PCL composite fibrous scaffolds. J Biomed Mater Res Part B: Appl Biomater 72B:156-65.

Zhao S, Wu X, Wang L, Huang Y. 2004. Electrospinning of ethyl-cyanoethyl cellulose/tetrahydrofuran solutions. J Appl Polym Sci 91(1):242-6.

Zhu QY, Zhang A, Tsang D, Huang Y, Chen Z-Y. 1997. Stability of green tea catechins. J Agric Food Chem 45:4624-8.

Zimeri J, Tong $\mathrm{CH} .1999$. Degradation kinetics of (-)-epigallocatechin gallate as a function of $\mathrm{pH}$ and dissolved oxygen in a liquid model system. J Food Sci 64(5):753-8.

Zong X, Kim K, Fang D, Ran S, Hsiao BS, Chu B. 2002. Structure and process relationship of electrospun bioabsorbable nanofiber membranes. Polymer 43:4403-12. 


\section{APPENDICES}

\subsection{Calibration Curve of EGCG Degradation}

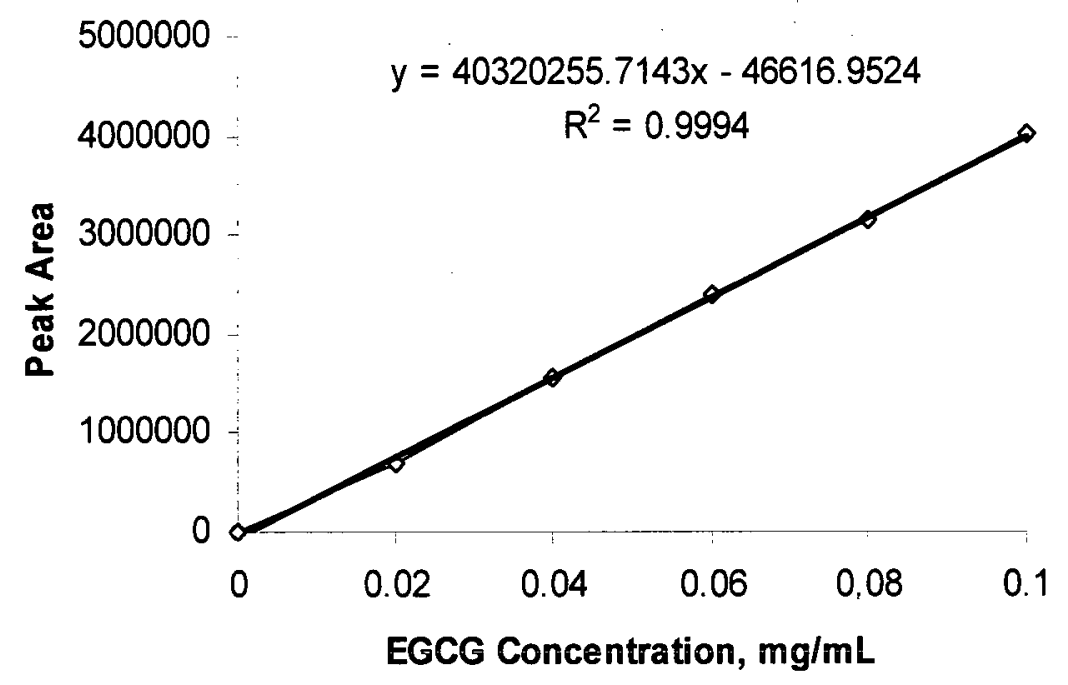

Figure 33 - Calibration curve of EGCG standard was linear over the range of $0.02-0.1 \mathrm{mg} / \mathrm{mL}$.

\subsection{Calibration Curve of GCG Degradation}

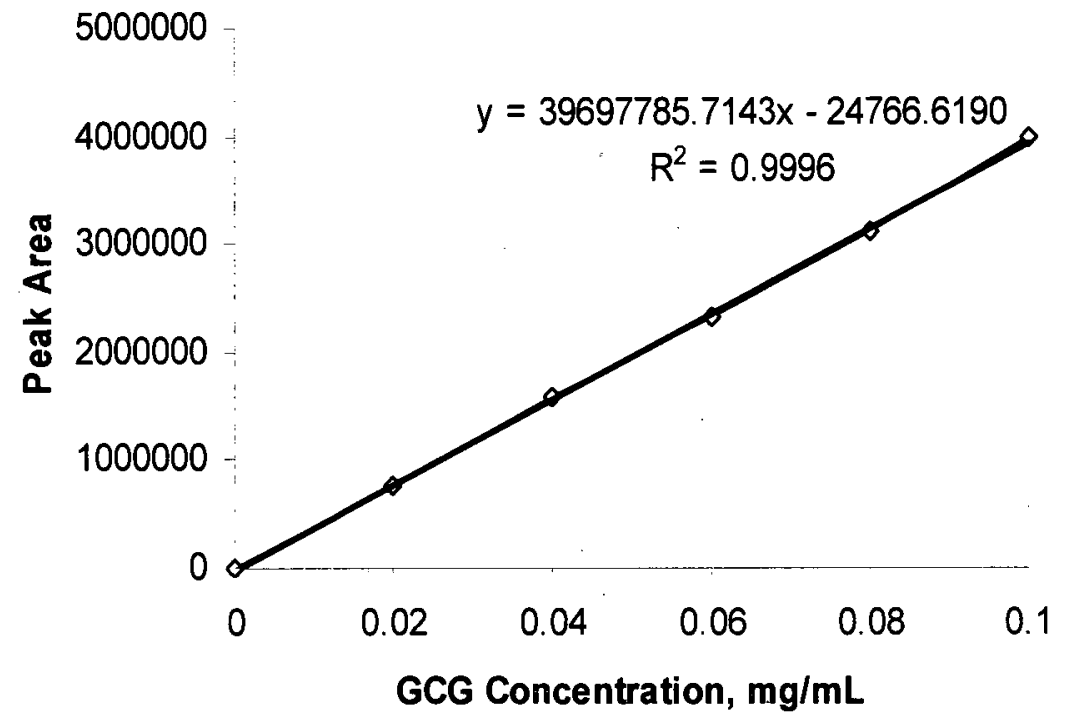

Figure 34 - Calibration curve of GCG standard. 


\subsection{Calibration Curve of EGC Degradation}

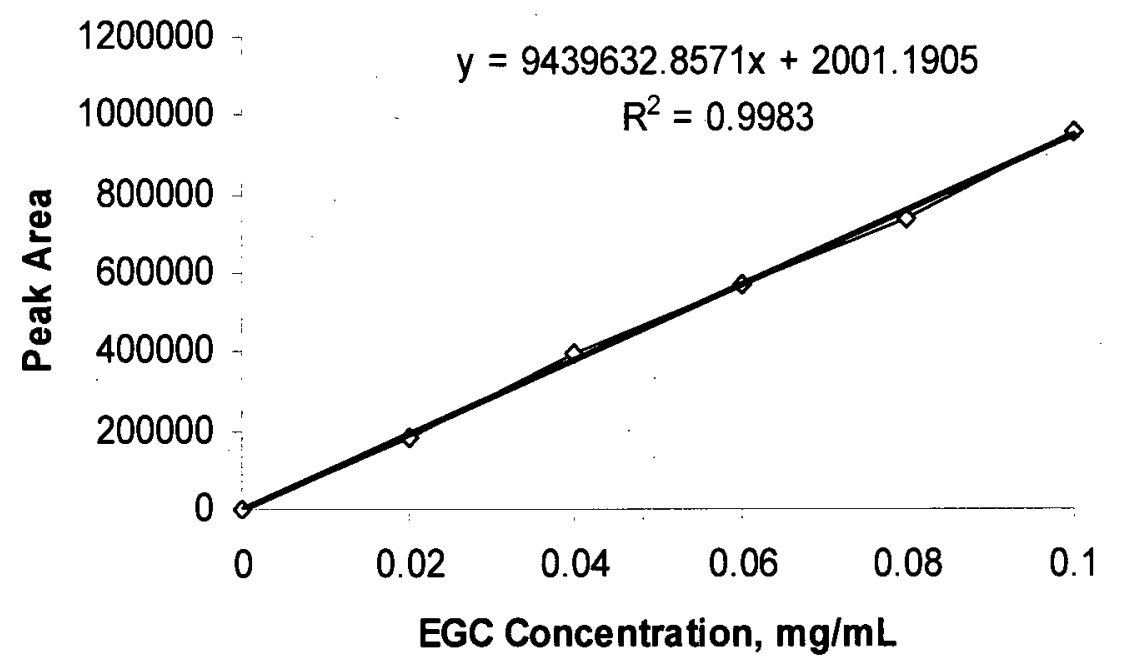

Figure 35 - Calibration curve of EGC standard.

\subsection{Matlab Program for model fitting and rate constant determination}

Matlab 7.0 applying non-linear regression was used to model the degradation reaction kinetics and estimate the rate constants for epimerization $\left(k_{1}\right)$, degradation $\left(k_{y i}\right)$ and diffusion $\left(k_{2}\right)$ of EGCG encapsulated in fibers. In the M-file programming, the function equation is shown as followed,

$$
y_{i}=E G C G(\text { beta }, x)
$$

beta and $x$ are the variables, representing a series of rate constants $\mathrm{k}$ and time $\mathrm{t}$ respectively. It is assumed that $\operatorname{beta}(1)$ is equal to the total values of $k_{l}+k_{2}+k_{y l}$, therefore the equation 7.14 is simplified to

$$
y_{i}=100 * \exp (-\operatorname{beta}(1) * x)
$$


The detailed programming output for the model fitting and rate constants determination for treatments in aqueous solution with $\mathrm{pH} 7$ at $100{ }^{\circ} \mathrm{C}$ as an example is described as following below,

>> yi7100

$y=$

$\begin{array}{lllllll}100.0000 & 67.9800 & 47.4500 & 23.2600 & 1.7400 & 0.0200 & 0\end{array}$

$x=$

$\begin{array}{lllllll}0 & 3 & 5 & 10 & 30 & 60 & 130\end{array}$

$>>$ beta0 $=[0,0]$

beta0 $=$

$0 \quad 0$

>> [beta,r,J]=nlinfit(x,y,'EGCGyi', beta0)

beta $=$

0.1627

0

$r=$

0

2.7978

$-1.5522$

$-0.7521$

0.3555

0.0008

$-0.0000$

$\mathrm{J}=$

$0 \quad 0$

$-195.5466 \quad 0$

$-245.01080$ 


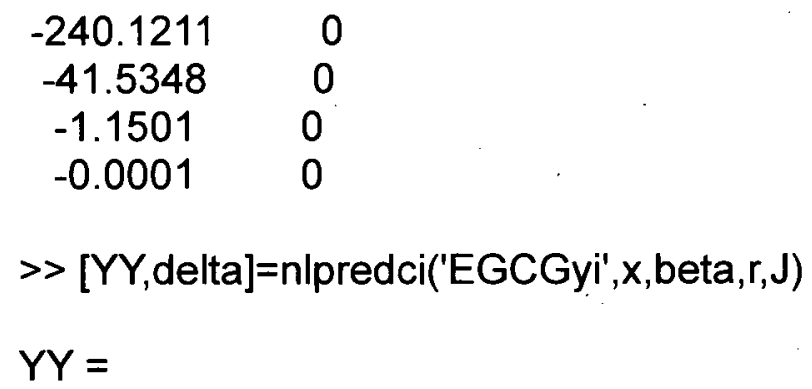


Output is,

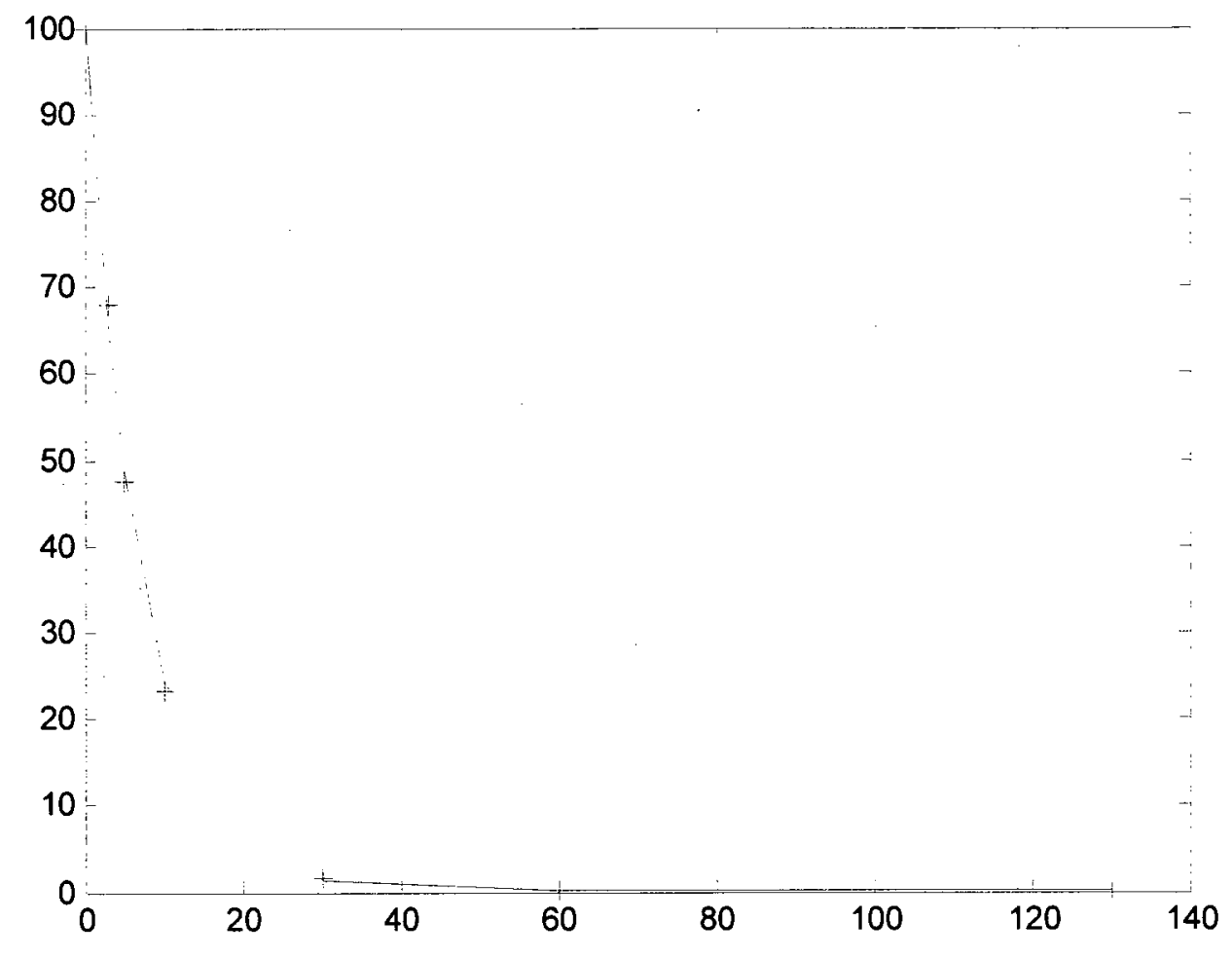

Matlab 7.0 applying non-linear regression was used to model the degraded GCG and EGC from EGCG encapsulated in fibers in the same way as the programming as described above. To estimate the rate constants for $k_{l}, k_{y i}$ and $k_{2}$, $k_{l}+k_{2}+k_{y i}$ is 0.1627 resulting from the output example. Substitute it to Equation 7.15 , following on

$$
y_{o}=\frac{100 k_{2}}{\left(k_{2}+k_{y i}\right)-0.1627}\left[\exp (-0.1627 * t)-\exp \left(-\left(k_{2}+k_{y i}\right) t\right)\right]
$$

and assuming beta(2) represents $k_{2}$ and beta(3) equals to $k_{2}+k_{y i}$ in the modelling programming, and substituting them into Equation 10.3 as shown as followed, 


$$
y_{o}=\frac{100 * \operatorname{beta}(2)}{\operatorname{beta}(3)-0.1627}[\exp (-0.1627 * t)-\exp (-\operatorname{beta}(3) * t)]
$$

(Equation 10.4)

After running the program by applying Equation 10.4, as a result, $k_{2}$ is 0.0785 and $k_{2}+k_{y i}$ equals to 0.1049 , then $k_{l}$ and $k_{y i}$ come out to 0.0578 and 0.0264 , respectively.

And then, to ensure the correct values, it is assumed that beta(4) and beta(5) represent $k_{1}$ and $k_{1}+k_{2}$, respectively, by inputting total value of $k_{1}+k_{2}+k_{y i}$ into Equation 7.16, resulting as shown in Equation 10.5,

$$
z_{i}=\frac{100 * \operatorname{beta}(4)}{\operatorname{beta}(5)}[\exp (0.0578)-\exp (-0.1627 * t)]
$$

Finally, the rate constants for $k_{1}, k_{2}$ and $k_{y i}$ for treatments in aqueous solution with $\mathrm{pH}$ at $100^{\circ} \mathrm{C}$ are estimated, and results are shown below,

$$
\left\{\begin{array}{l}
k_{1}=0.0578 \\
k_{2}=0.0785 \\
k_{y i}=0.0264
\end{array}\right.
$$

The estimations for the resulting rate constants of all the treatments shown in Figure 28 - 31 were followed the same procedure as described as above. 


\subsection{Activation energy}

The rate constant $k$ is affected by the temperature and this dependence may be represented by the Arrhenius Equation:

$$
k=A \exp \left(-E_{a} / R T\right)
$$

where the pre-exponential factor $A$ is assumed to be independent of temperature, $R$ is the gas constant, and $T$ the temperature in $K$. Taking the natural logarithm of this equation gives:

$$
\ln k=\ln A-E_{a} /(R T)
$$

rearranging it, when $C$ represents a constant,

$$
\ln k=-\left(E_{a} / R\right) *(1 / T)+C
$$

These equations indicate that the plot of $\ln k$ vs. $1 / T$ is a straight line, with a slope of $-E_{d} / R$. These equations provide the basis for the experimental determination of $E_{a}$. To calculate the energy activations for all the treatments in this study, the procedure for treatment in aqueous solution with $\mathrm{pH} 7$ at various temperatures as an example is described as followed,

As resulted from the previous section, the rate constant for epimerization $\left(k_{l}\right)$ is determined at $277 \mathrm{~K}$ and $373 \mathrm{~K}$ are 0.00005748 and 0.05780067 , respectively. Let $k_{1}$ and $k_{2}$ be the rate constants determined at $T_{1}$ and $T_{2}$, respectively. Then substitute them into two equations: 


$$
\begin{aligned}
& \ln k_{1}=\ln A-E_{a} /\left(R T_{1}\right) \\
& \ln k_{2}=\ln A-E_{a} /\left(R T_{2}\right)
\end{aligned}
$$

From these, you should be able to derive the following relationships,

$$
\ln \frac{k_{2}}{k_{1}}=-\frac{E_{a}}{R}\left(\frac{1}{T_{2}}-\frac{1}{T_{1}}\right)
$$

Further, it is given,

$$
\begin{aligned}
E_{a} & =\frac{T_{1} * T_{2} * R}{T_{2}-T_{1}} * \ln \frac{k_{2}}{k_{1}} \\
& =\frac{(373 \mathrm{~K}) *(277 \mathrm{~K}) * 8.314472 \mathrm{~J} /(\mathrm{K} * \mathrm{~mol})}{(373-277) \mathrm{K}} * \ln \frac{0.05780067}{0.00005748} \\
& =61.86 \mathrm{KJ} / \mathrm{mol}
\end{aligned}
$$

Therefore, the corresponding $E_{a}$ for the epimerization of EGCG encapsulated in fibers is $61.86 \mathrm{KJ} / \mathrm{mol}$. The same procedures for estimations of energy activations were applied to all the treatments. 


\subsection{Stability Data}

Table 3 - Remaining percentage of residual EGCG encapsulated by electrospun zein fibers in aqueous system with $\mathrm{pH} 3-9$ at different temperature, compared to the percentage of controls.
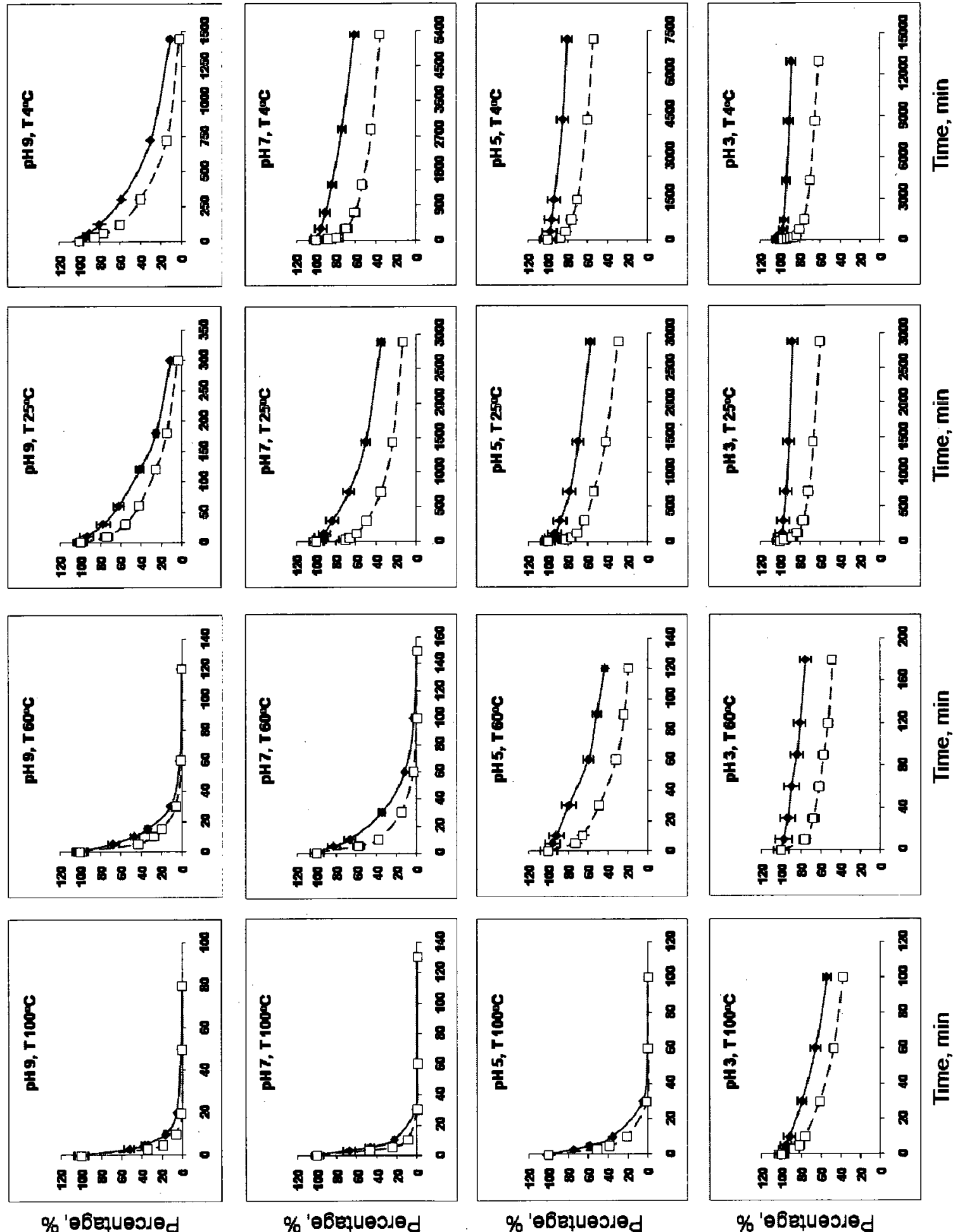

$\%$ 'erbiueded

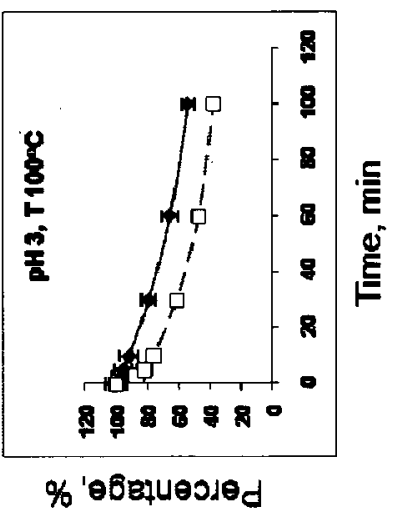


Table 4 - Remaining percentage of epimerized GCG from EGCG encapsulated by electrospun zein fibers in aqueous system with $\mathrm{pH} 3-9$ at different temperature, compared to the percentage of GCG epimerized from EGCG controls.
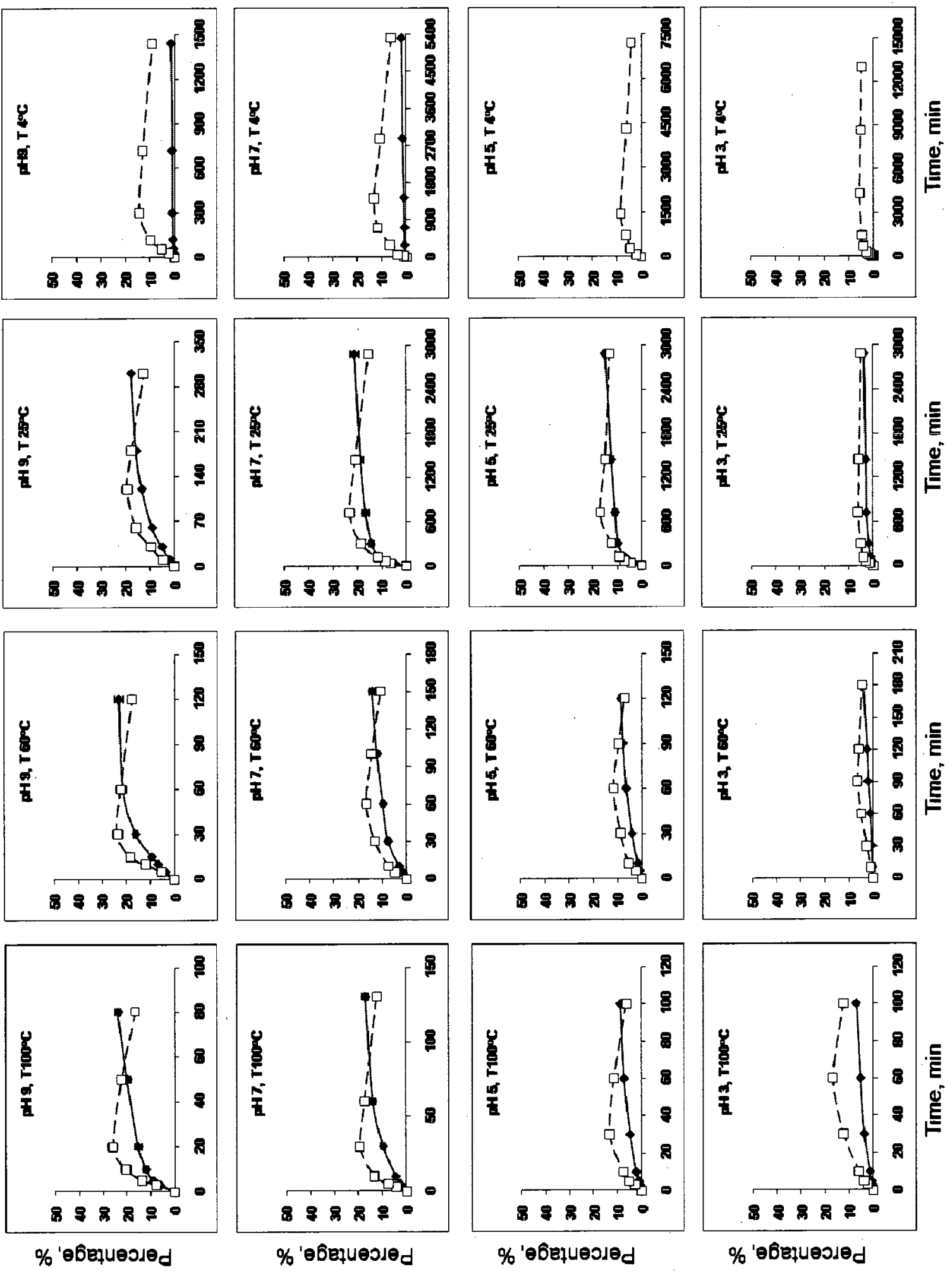
Table 5 - Remaining percentage of degraded EGC from EGCG encapsulated by electrospun zein fibers in aqueous system with $\mathrm{pH} 3-9$ at different temperature, compared to the percentage of EGC degraded from EGCG controls.
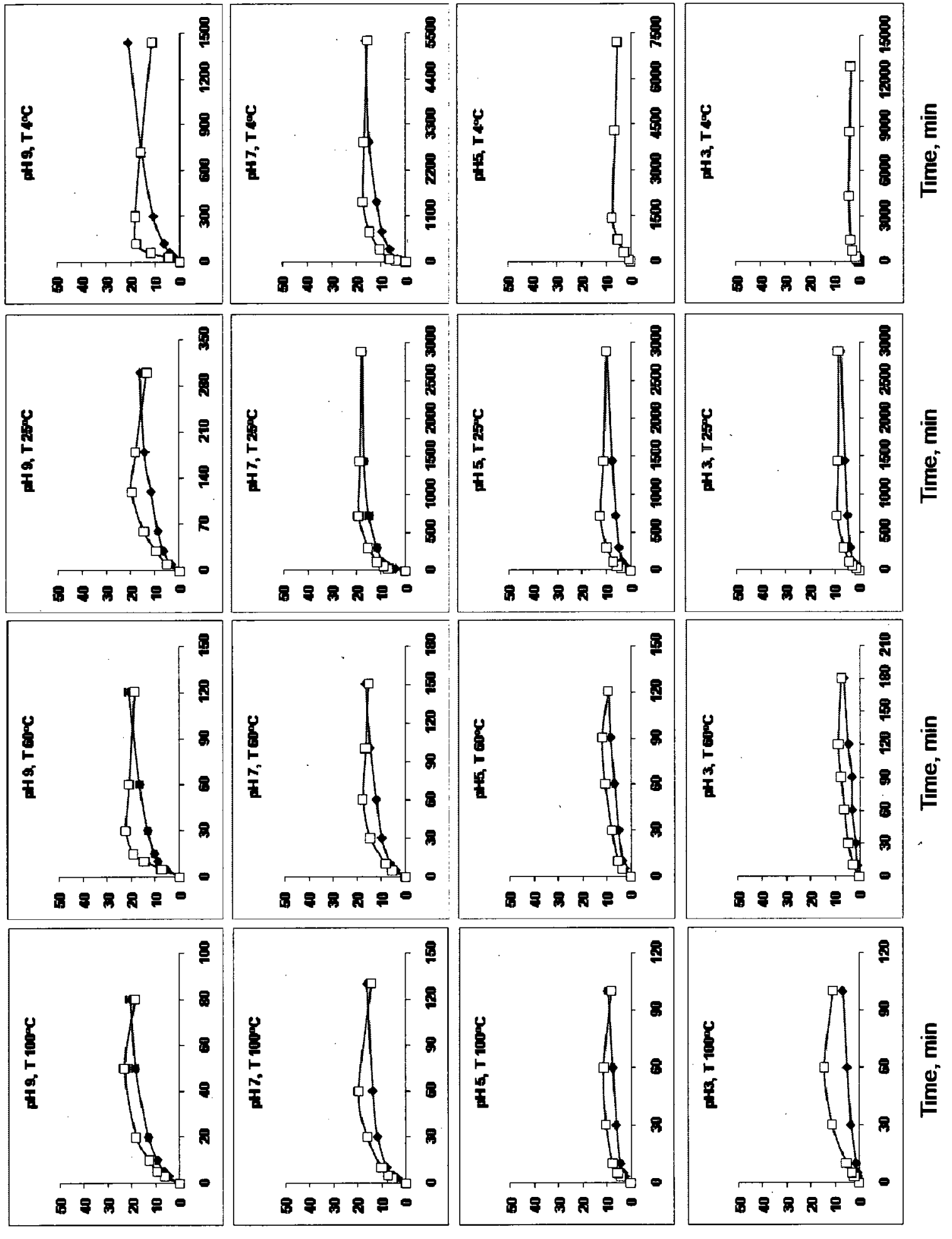

\%'ebequejed

$\%$ 'өbiłuejed

\% 'өbequeosed

\%'ebequeded 